INSTITUTE

FOR ADVANCED

STUDIES

LUCCA

IMT LUCCA CSA TECHNICAL

REPORT SERIES 01

July 2012

Updated on November 2012

\title{
A Uniform Definition of Stochastic Process Calculi
}

Rocco De Nicola

Diego Latella

Michele Loreti

Mieke Massink 
IMT LUCCA CSA TECHNICAL REPORT SERIES \#01/2012

(C) IMT Institute for Advanced Studies Lucca

Piazza San Ponziano 6, 55100 Lucca

Research Area

Computer science and applications

\section{A Uniform Definition of Stochastic Process Calculi}

Rocco De Nicola

IMT Institute for Advanced Studies Lucca

Diego Latella

CNR - Istituto di Scienza e Tecnologie dell'Informazione "A. Faedo", Pisa

Michele Loreti

Università di Firenze - Dipartimento di Sistemi e Informatica

\section{Mieke Massink}

CNR - Istituto di Scienza e Tecnologie dell'Informazione "A. Faedo", Pisa 


\title{
A Uniform Definition of Stochastic Process Calculi
}

\author{
ROCCO DE NICOLA \\ IMT - Institute for Advanced Studies, Lucca \\ DIEGO LATELLA \\ CNR - Istituto di Scienza e Tecnologie dell'Informazione "A. Faedo", Pisa \\ MICHELE LORETI \\ Università di Firenze - Dipartimento di Sistemi e Informatica, Firenze \\ and \\ MIEKE MASSINK \\ CNR - Istituto di Scienza e Tecnologie dell'Informazione "A. Faedo", Pisa
}

\begin{abstract}
We introduce a unifying framework to provide the semantics of process algebras, including their quantitative variants useful for modeling quantitative aspects of behaviors. The unifying framework is then used to describe some of the most representative stochastic process algebras. This provides a general and clear support for an understanding of their similarities and differences. The framework is based on State to Function Labeled Transition Systems, FuTSs for short, that are state-transition structures where each transition is a triple of the form $(s, \alpha, \mathscr{P})$. The first and the second components are the source state, $s$, and the label, $\alpha$, of the transition, while the third component is the continuation function, $\mathscr{P}$, associating a value of a suitable type to each state $s^{\prime}$. For example, in the case of stochastic process algebras the value of the continuation function on $s^{\prime}$ represents the rate of the negative exponential distribution characterizing the duration/delay of the action performed to reach state $s^{\prime}$ from $s$. We first provide the semantics of a simple formalism used to describe Continuous-Time Markov Chains, then we model a number of process algebras that permit parallel composition of models according to the two main interaction paradigms (multiparty and one-to-one synchronization). Finally, we deal with formalisms where actions and rates are kept separate and address the issues related to the co-existence of stochastic, probabilistic, and non-deterministic behaviors. For each formalism, we establish the formal correspondence between the FUTSs semantics and its original semantics.
\end{abstract}

Categories and Subject Descriptors: F.1.1 [COMPUTATION BY ABSTRACT DEVICES]: Models of Computation; G.3 [PROBABILITY AND STATISTICS]: ; G.2.1 [SOFTWARE ENGINEERING]: Requirements/Specifications

General Terms: Languages, Performance, Theory, Verification

Additional Key Words and Phrases: Continuous Time Markov Chains, Stochastic Process Calculi, Structured Operational Semantics

Corresponding Author's address: Rocco De Nicola, IMT - Institute for Advanced Studies, Lucca I-55100 - Lucca ITALY; email: rocco.denicola @imtlucca.it. This work has been partially funded by EU Collaborative project $\mathrm{n}$. 257414 ASCENS, and Project TRACE-IT - PAR FAS 2007-2013 - Regione Toscana

Permission to make digital/hard copy of all or part of this material without fee for personal or classroom use provided that the copies are not made or distributed for profit or commercial advantage, the ACM copyright/server notice, the title of the publication, and its date appear, and notice is given that copying is by permission of the ACM, Inc. To copy otherwise, to republish, to post on servers, or to redistribute to lists requires prior specific permission and/or a fee.

(C) 2012 ACM 0000-0000/2012/0000-0001 $\$ 5.00$ 


\section{INTRODUCTION}

Distributed computing has become a ubiquitous technology, mainly thanks to the infrastructure of the global Internet. Open-ended, highly parallel, massively distributed systems will soon span over millions of nodes showing complex interactions and behaviors. This will make it increasingly difficult to develop, deploy, and manage such systems, and may lead to increasing management costs and use of resources. It is thus of paramount importance to find ways that guarantee such systems behave reliably and predictably. Formal models for describing systems, and for reasoning about their behavior in open-ended, nondeterministic environments, offer a well-established approach for such guarantees.

Process Algebras (see [Bergstra et al. 2001] and the references therein) have been successfully used over the last thirty years to model and analyze the behavior of concurrent distributed systems. They are based on mathematically rigorous process description languages with well-defined semantics that provide models of processes, regarded as agents that perform actions (act) and communicate (interact) with similar agents and with their environment.

To define a process description language at the base of a process algebra, one starts with a set $\mathcal{A}$ of uninterpreted action names (that might represent communication channels, synchronization actions, etc.) and with a set of elementary processes that, together with the actions, are the basic building blocks for forming new processes from existing ones. The basic operators are: action prefix, (a.- - modeling execution of a generic action $a$, followed by the remaining behavior), non-deterministic choice _ $_{+}^{+}{ }_{-}$- modeling abstract conditional branches) and parallel composition (_ $\|$ _ - modeling concurrent execution). There is a set of axioms and inference rules for each operator, which form the basis for defining the behavior of a composite system in terms of the behavior of its components, assembled by using the given operator. This is the core of a compositional definition of the semantics of a language. In this way, a labeled graph can be associated to each term of the language whose states are the terms themselves and whose labels are the actions that can be performed from each state. This particular class of labeled graphs is known as Labeled Transition Systems (LTSs).

Process Description Languages (PDLs) often come equipped with observational mechanisms that permit equating (through behavioral equivalences) those systems that cannot be distinguished by external observations in order to have the possibility of replacing a system with an equivalent one whenever needed. LTSs, possibly corresponding to process terms describing systems at different levels of abstraction, are then compared according to a behavioral relation [van Glabbeek 2001], giving rise to so-called Process Calculi (PCs). In some cases, the behavioral relations also have complete axiomatizations, in forms of equations, that exactly capture the relevant equivalences induced by the abstract operational semantics; then PCs are also called Process Algebras (PAs). Nowadays, PA, PC and PDL are often used interchangeably. In the context of the present paper, we will use the acronym 'PC'.

Initially, process calculi were mainly designed to model functional (extensional) system behavior. However, it was soon recognized that, in order to capture other important features of concurrent systems, variants were needed to take quantitative features into account. This led to the development of timed process calculi, probabilistic process calculi, and stochastic(ally timed) process calculi. The latter have proven to be particularly suitable for capturing important properties related to performance and quality of service, and even for 
the modeling of biological systems.

Terms of Stochastic Process Calculi (SPCs) can be used to generate Continuous-Time Markov Chains (CTMCs), or Interactive Markov Chains (IMCs), that permit quantitative system analyses. Both CTMCs and IMCs consider delays as Random Variables (RVs) with negative exponential distributions; each of them is thus characterized by its unique parameter, the rate $\lambda>0$, and has expected value $\lambda^{-1}$. CTMC-based proposals associate time with actions, annotating them with rates. IMCs have instantaneous actions and an additional transition relation, specifically introduced to model the passage of time. CTMCs model fully stochastic behavior of systems, i.e. they choose between possible futures by relying exclusively on the concept of race condition, while IMCs describe stochastic behaviors intertwined with explicitly modeled non-deterministic choices. The approach leading to CTMCs is employed by the vast majority of the proposals in the literature. In this approach rates are associated with actions by means of a rated action prefix $\langle a, \lambda\rangle . P$, with $\lambda$ being the rate associated with $a$. In IML [Hermanns 2002], the calculus for IMCs, nondeterminism is, instead, not banned and rates are used to characterize process delays, by using rate prefix $\lambda . P$.

Finally, in Markov Automata, each action is followed by a discrete probability (sub)distribution over processes, rather than by a single process, while delays are modeled by exponentially distributed RVs, as usual. Thus, a third dimension, namely process probability distributions, is introduced besides non-determinism and stochastic continuous time (see e.g. [Eisentraut et al. 2010]). However, even if one restricts the focus of attention to fully stochastic calculi leading to CTMCs (i.e. those calculi where non-deterministic behavior is completely replaced by a probabilistic one, thanks to stochastic time), the models underlying their definition are significantly different in many respects. Some differences are conceptual. For instance, multi-party process synchronization is used in most SPCs, although there are notable examples of one-to-one process synchronization use, like stochastic $\pi$-calculus [Priami 1995] and stochastic CCS [Klin and Sassone 2008].

Other differences, instead, are purely technical, in the sense that they stem from different approaches to addressing the same concept. A prominent example of such a technical difference is the modeling of the race condition principle and its relationship to the issue of transition multiplicity. This principle implies that, for a generic process $P$, an expression like $\langle a, \lambda\rangle . P+\langle a, \lambda\rangle . P$ is intended to model the same behavior as $\langle a, 2 \lambda\rangle . P$, as the exit rate of the (random) sojourn time in state $\langle a, \lambda\rangle . P+\langle a, \lambda\rangle . P$ is $2 \cdot \lambda$. Unfortunately, if one takes a standard LTS interpretation, it holds that $\langle a, \lambda\rangle . P+\langle a, \lambda\rangle . P$ and $\langle a, \lambda\rangle . P$ yield the very same systems. This problem has implicated the need to take transition multiplicity into account and accordingly several, significantly different, approaches have been proposed for handling it. They range from the use of multi-relations [Hillston 1996; Hermanns 2002] to proved transition systems [Priami 1995; Gotz et al. 1993] and from LTSs with numbered transitions [Hermanns et al. 2002] to unique rate names [De Nicola et al. 2005; De Nicola et al. 2006], to mention just a few ${ }^{1}$. The feature that unites them all is that they require two steps to obtain the 'right' rate: first an enriched LTS is built and then it is manipulated to properly add up rates.

In order to provide a uniform account of different fully stochastic calculi, in [De Nicola

${ }^{1}$ It should, however, be noticed that, due to a minor technical imprecision (multi-relations are defined as the least multi-relation satisfying a set of SOS axioms and rules) both [Hillston 1996] and [Hermanns 2002] obtain relations, and not the intended multi-relations, thus failing to model transition multiplicity. 
et al. 2009c], we have proposed a variant of LTSs, namely Rate Transition Systems (RTSs). This model is inspired by the approach taken when modeling probabilistic systems via probabilistic automata, where operators derived from those of the process calculi are applied to probability distributions, as e.g. in [Deng et al. 2007]. In LTSs, a transition is a triple $\left(P, \alpha, P^{\prime}\right)$ where $P$ is the source state, $\alpha$ is the label of the transition, and $P^{\prime}$ is the target state reached from $P$ via $\alpha$. On the other hand, in RTSs a transition is a triple of the form $(P, \alpha, \mathscr{P})$, whose first and second component are again the source state and the transition label, but the third component $\mathscr{P}$ is the continuation function that associates a real non-negative value with each state $P^{\prime}$. A non-zero value represents the rate of the exponential distribution characterizing the time needed for the execution of the action represented by $\alpha$, necessary to reach $P^{\prime}$ from $P$ via the transition. Whenever $\mathscr{P}\left(P^{\prime}\right)=0, P^{\prime}$ is not reachable from $P$ via $\alpha$. RTS continuation functions are equipped with a rich set of operations which provide a simple and clean solution to the transition multiplicity problem. Moreover, they make RTSs particularly suitable as a framework for the compositional definition of fully stochastic calculi. Indeed, the transition system with the 'right' rates is built directly rather than relying on the two steps mentioned above. Finally, it must also be noticed that continuation functions provide the minimal amount of information needed to characterize the underlying CTMC. In fact, the exit rate of a state $P$ as well as the probability of jumping to a state $P^{\prime}$ can be easily computed with continuation functions. For instance, assuming that for each $P$ and $\alpha$ there is a unique transition $(P, \alpha, \mathscr{P})$, as will be shown to be the case for all major SPCs, the $\alpha$-exit-rate from state $P$ is just $\sum_{P^{\prime}}\left(\mathscr{P} P^{\prime}\right)$ while the probability of reaching a process $Q$ after a $\alpha$ is simply $\frac{\mathscr{P}(Q)}{\sum P^{\prime}\left(\mathscr{P} P^{\prime}\right)}$, if the $\alpha$-exit-rate is positive. The total exit rate for $P$ is then $\sum_{\alpha} \sum_{P^{\prime}}\left(\mathscr{P}_{\alpha} P^{\prime}\right)$ where, for given $P, \mathscr{P}_{\alpha}$ is the unique continuation function such that $\left(P, \alpha, \mathscr{P}_{\alpha}\right)$.

RTSs are similar to Rated Transition Systems (Rated TSs), introduced by Klin and Sassone [2008]. A Rated TS is induced by a Stochastic Generalized SOS (SGSOS) specification $^{2}$ and is characterized by a function $\rho$ such that $\rho\left(P, \alpha, P^{\prime}\right) \in \mathbb{R}_{\geq 0}$ is the rate associated to the $\alpha$-transition from $P$ to $P^{\prime}$, when positive. This is obviously equivalent to having a function $\rho(P, \alpha, \cdot)$ which associates a real, non negative, number to each $P^{\prime}$, which in turn means that Rated TSs are a form of "rate-deterministic" RTSs in the sense that for each $P$ and $\alpha$ there exists exactly one transition $(P, \alpha, \mathscr{P})$.

In the present paper, we introduce State to Function Labeled Transition Systems, FuTSs for short, a generalization of RTSs [De Nicola et al. 2009c] based on a simple generalization of the co-domain of the continuation functions, which enables us to consider a richer class of models in addition to being able to take non-deterministic systems into account. FuTSs have generic commutative semi-rings, and not just the set of non-negative reals, as co-domain of continuation functions. Furthermore, in the general case, we let the third component of a transition be an element of a disjoint union of sets of continuation functions, with different co-domains, so that different 'kinds' of transitions can be modeled. The semi-ring structure of the co-domain preserves basic properties of primitive operations like sum and multiplication, which prove very useful when modeling composition of rates resulting from (parallel, non-deterministic, sequential) process compositions. Continuation functions are equipped with a rich set of (generic) operations, making FuTSs very well suited as a semantic domain for the compositional definition of the operational semantics of process calculi. Such operations induce an algebraic structure on the set of

${ }^{2}$ SGSOS are essentially the analogous, in the context of SPCs, of GSOS [Aceto et al. 2001].

ACM Computing Surveys - In Press, Vol. , No., November 2012. 
continuation functions, which we systematically exploit for the compositional definition of the FuTS semantics of SPCs. FuTSs thus support a uniform and systematic understanding of similarities and differences of the many stochastic calculi proposed in the literature.

In the remainder of this article, while we address most of the calculi mentioned above, we will focus on fragments which are relevant for their stochastic features. Our selection of calculi has been guided by the aim to provide a survey of most of the notions that have been considered thus far. We first consider a simple language for CTMCs, then we will consider different calculi that permit parallel composition of models according to the two main communication paradigms (multi-party and one-to-one synchronization). Finally, we will consider languages where actions and rates are kept separate, then addressing the issues related to non-determinism and discrete probabilities by showing how IMCs and Markov Automata can be represented by FuTSs.

We would like to remark that, in the present article, we do not aim at presenting a systematic study of, and comparison between, all of the stochastic calculi that have been proposed in the literature (the interested reader is referred to, e.g. [Aldini et al. 2010; Hermanns et al. 2002; Brinksma and Hermanns 2001]), nor do we attempt to investigate the pros and cons of the various approaches regarding the definition of the rates of synchronizing actions and related pragmatics (see, e.g. [Hillston 1994; Bernardo 2010]). Rather, we aim at showing how the main techniques used to describe their semantics can be accommodated within a common simple unifying framework that offers the reader a uniform account of the many stochastic calculi proposed in the literature. This facilitates the appreciation of similarities and differences between the many formalisms and can guide the choice of appropriate stochastic modeling tools.

The paper is organized as follows: in Sect. 2 some preliminary notions and definitions are recalled and FuTSs are introduced; examples of how other structures like CTMCs, Discrete Time Markov Chains (DTMCs), RTSs and Weighted Transition Systems [Fahrenberg et al. 2011] can be represented using FuTSs are presented as well. In Sect. 3 the process operators which will be used in subsequent sections for the specific stochastic calculi are presented and briefly discussed. Sect. 4 introduces the FuTS semantics of a simple language for CTMCs. Sect. 5 addresses the issue of introducing parallel composition in the FuTS framework, thus paving the way for the next sections. Sect. 6 shows the FuTS semantics of significant fragments of CSP-like calculi based on the multi-party interaction paradigm. The FuTS semantics of CCS-like calculi is presented in Sect. 7. The issue of coexisting stochastic, probabilistic and non-deterministic behaviors in the FuTS framework is addressed in Sect. 8. Related work is discussed in Sect. 9 while in Sect. 10 we draw some concluding remarks. The original SOS definition of the relevant calculi as well as detailed proofs are reported in the appendices.

\section{PRELIMINARIES}

In this section we first recall the notions of continuous time Markov chain and of labelled transition system, then we introduce commutative semi-rings and state to function labelled transition systems.

\subsection{Continuous Time Markov Chains}

A CTMC is characterized by a set of states and by a rate matrix. The latter identifies system transitions in terms of a set of exponentially distributed Random Variables (RVs). 
Definition 2.1. A real valued RV $D$ has a negative exponential distribution with rate $\lambda \in \mathbb{R}_{>0}$ iff the probability that $D \leq t \in \mathbb{R}(\mathbb{P}\{D \leq t\})$ is $1-e^{-\lambda \cdot t}$ for $t>0$, is 0 otherwise.•

The expected value of an exponentially distributed RV with rate $\lambda$ is $\lambda^{-1}$ while its variance is $\lambda^{-2}$. Given the exponentially distributed independent $\mathrm{RVs} D_{1}, \ldots, D_{n}$ with rates $\lambda_{1}, \ldots, \lambda_{n}$ respectively, the $\mathrm{RV} \operatorname{MIN}\left\{D_{1}, \ldots, D_{n}\right\}$ is exponentially distributed with rate $\lambda=\lambda_{1}+\ldots+\lambda_{n}$, while $\mathbb{P}\left\{D_{j}=\operatorname{MIN}\left\{D_{1}, \ldots, D_{n}\right\}\right\}=\frac{\lambda_{j}}{\lambda_{1}+\ldots+\lambda_{n}}$.

Definition 2.2. A continuous time Markov chain (CTMC) is a tuple $(S, \mathbf{R})$ where $S$ is a countable non-empty set of states, and $\mathbf{R}: S \rightarrow S \rightarrow \mathbb{R}_{\geq 0}$ is the rate matrix, where for all $s \in S: \sum_{s^{\prime} \in S} \mathbf{R} s s^{\prime}<\infty$.

We will often use the matrix notation $\mathbf{R}\left[s, s^{\prime}\right]$ for $\mathbf{R} s s^{\prime} . \mathbf{R}\left[s, s^{\prime}\right]>0$ means that a transition from $s$ to $s^{\prime}$ can be taken, while no transition from $s$ to $s^{\prime}$ exists if $\mathbf{R}\left[s, s^{\prime}\right]=0$. The sojourn time at state $s$ before taking a transition is an exponentially distributed RV with rate $\sum_{s^{\prime} \in S} \mathbf{R}\left[s, s^{\prime}\right]$ and the probability that the transition from $s$ to $s^{\prime}$ is taken is $\frac{\mathbf{R}\left[s, s^{\prime}\right]}{\sum_{s^{\prime \prime} \in S} \mathbf{R}\left[s, s^{\prime \prime}\right]}$.

Notice that the above definition allows $\mathbf{R}[s, s]>0$, i.e. CTMCs may have self-loops. This is different from the, more traditional, characterization of CTMCs by their infinitesimal generator matrix, which is only unique up to self-loops, meaning that CTMCs which differ only in self-loops will have the same generator matrix, and the same probability distributions at both transient and steady state. Thus, the above, more liberal definition of CTMCs does not affect their meaning as long as traditional measures like transient and steady state probabilities are concerned (see [De Nicola et al. 2009b] for details).

\subsection{Labelled Transition Systems}

Formal semantics of SPCs are traditionally defined by means of Structured Operational Semantics (SOS) that lead to Labelled Transition Systems (LTSs), i.e. structures of the form $(S, A, \rightarrow)$, where $S$ is a set of states, $A$ a set of labels and $\rightarrow \subseteq S \times A \times S$ is the transition relation [Plotkin 2004].

The states of the LTS are the terms of the language, while the actual transition relation is defined by means of a logical deduction system as shortly sketched in the following. The rules of the deduction system are statements of the form $P \stackrel{a}{\rightarrow} Q$, where $P$ and $Q$ are placeholders for generic terms of the language generated by a BNF-like grammar. The transition relation is defined by inducing on such grammar, using rules of the form

$$
\frac{\text { Premise }_{1}, \ldots, \text { Premise }_{n}}{\text { Conclusion }}
$$

meaning that 'Conclusion' can be drawn from the premises 'Premise ${ }_{1}$ ' to 'Premise ${ }_{n}$ '. Rules without premises are axioms. Normally, there are one or more rules for each operator of the language that have the latter as top-level constructor on the left hand side component in the conclusion; the definition of the semantics is thus directed by the syntax of the language.

Consider, for example, the simple language with only action prefix, choice and the null process defined by the grammar below, where $a \in \mathcal{A}$ for some action set $\mathcal{A}$ :

$$
P::=\text { nil } \mid \text { a.P } \mid P+P
$$

The SOS of the above language is composed of one axiom (for action prefix) and two rules (for choice), as in Fig. 1. The axiom (AP) states that $a . P$ performs $a$ and then behaves like $P$, while rules $(\mathrm{CH} 1)$ and $(\mathrm{CH} 2)$ state that $P+Q$ may behave either like $P$ or like $Q$. Using the rules of Fig. 1, one can deduce, for example, that the transitions of the LTS for term 


$$
\text { (AP) } \frac{P . P \stackrel{a}{\longrightarrow} P}{a .} \quad(\mathrm{CH} 1) \frac{P \stackrel{a}{\longrightarrow} P^{\prime}}{P+Q \stackrel{a}{\longrightarrow} P^{\prime}} \quad(\mathrm{CH} 2) \frac{Q \stackrel{a}{\longrightarrow} Q^{\prime}}{P+Q \stackrel{a}{\longrightarrow} Q^{\prime}}
$$

Fig. 1. Semantics Rules for a simple language

$a .(b$. nil $+c$. nil $)$, are the following: $a .(b$. nil $+c$. nil $) \stackrel{a}{\longrightarrow}(b$. nil $+c$. nil $),(b$. nil $+c$. nil $) \stackrel{b}{\longrightarrow}$ nil, $(b$. nil $+c$. nil $) \stackrel{c}{\longrightarrow}$ nil.

In SPCs, transitions are usually labelled by rates $\lambda$, and/or action labels. Sometimes, actions may also be associated with weights for capturing probability distributions. In this case, the associated transitions are labelled by actions and weights $\omega$. In some cases, the transition relation $\rightarrow$ is required to be a multi-set, in which case the LTS is called a Labelled Multi-transition System.

Henceforth, we let $\mathbf{r t}\left(P_{1}, P_{2}\right), \mathbf{r t}_{a}\left(P_{1}, P_{2}\right)$, and $\mathbf{w t}_{a}\left(P_{1}, P_{2}\right)$ denote the cumulative rate over all transitions from $P_{1}$ to $P_{2}$ and the cumulative rate and weight, respectively, over all $a$-labelled transitions, from $P_{1}$ to $P_{2}$. Formally: $\operatorname{rt}\left(P_{1}, P_{2}\right) \quad=\operatorname{def} \quad \sum\left\{\lambda \mid P_{1} \stackrel{\lambda}{\longrightarrow} P_{2}\right\}$, $\mathbf{r t}_{a}\left(P_{1}, P_{2}\right)==_{\text {def }} \sum\left\{\lambda \mid P_{1} \stackrel{a, \lambda}{\longrightarrow} P_{2}\right\}$, and $\mathbf{w t}_{a}\left(P_{1}, P_{2}\right)==_{\text {def }} \sum\left\{\omega \mid P_{1} \stackrel{a, *_{\omega}}{\longrightarrow} P_{2}\right\}$ where $\{-\}$ denotes a multi-set, $\sum\{\}={ }_{\text {def }} 0$ and $\stackrel{\lambda}{\longrightarrow}, \stackrel{a, \lambda}{\longrightarrow}$ and $\stackrel{a, *_{\omega}}{\longrightarrow}$ identify a generic transition, an $a$-labelled transition with rate $\lambda$, an $a$-labelled transition with weight $\omega$, respectively.

In the context of stochastic extensions of CCS-like languages, proved transitions are used instead of transition multi-relations. Such transitions have an additional label encoding the derivation that provides a proof of the transition in a SOS deduction system and uniquely identifies the transition within the set of those coming out of a state. Letting $\theta, \theta^{\prime}$ be proof encodings, and $\mathcal{A}$ and $\overline{\mathcal{A}}$ be the sets of actions and co-actions respectively, for $a \in \mathcal{A} \cup \overline{\mathcal{A}}$, we define $\operatorname{rt}_{a}\left(P_{1}, P_{2}\right) \quad=_{\text {def }} \quad \sum\left\{\lambda \mid P_{1} \stackrel{\theta a, \lambda}{\longrightarrow} P_{2}\right\}$, and $\mathbf{r t}_{\langle a \| \bar{a}\rangle}\left(P_{1}, P_{2}\right)=_{\text {def }} \sum\left\{\lambda \mid P_{1} \stackrel{\left\langle\theta a, \theta^{\prime} \bar{a}\right\rangle, \lambda}{\longrightarrow} P_{2}\right\}$.

\subsection{Semirings: Notation and basic definitions}

We let $\mathbb{R}_{\geq 0}$ denote the set of real, non-negative numbers and, similarly, $\mathbb{R}_{>0}$ and $\mathbb{N}_{>0}$ denote the sets of positive real and natural numbers. $\mathbb{B}$ denotes the set of booleans. For any set $S$ we let $\wp S$ denote its power-set and $\wp_{\text {fin }} S$ the set of its finite subsets.

Many of the notions and definitions which we will use in the present paper are based on the concept of commutative semi-ring:

Definition 2.3. A semi-ring is a set $\mathbb{S}$ equipped with two binary operations, $+_{\mathbb{S}}$, called sum, and $\cdot \mathbb{S}$, called multiplication, such that: $\left(\mathbb{S},+_{\mathbb{S}}\right)$ is a commutative monoid with neutral element $0_{\mathbb{S}} \in \mathbb{S}$ and $(\mathbb{S}, \cdot \mathbb{S})$ is a monoid with neutral element $1_{\mathbb{S}} \in \mathbb{S}$. It is also assumed that multiplication distributes over sum and that $0_{\mathbb{S}}$ annihilates $\mathbb{S}$ with respect to multiplication. A semi-ring $\mathbb{S}$ is said to be commutative whenever $s_{1} \cdot \mathbb{S} s_{2}=s_{2} \cdot \mathbb{S} s_{1}$. Finally, we say that binary operation $/ \mathbb{S}$ is the inverse of $\cdot \mathbb{S}$ if $s_{3}=s_{1} /{ }^{\mathbb{S}} s_{2}$ if and only if $s_{1}=s_{2} \cdot \mathbb{S} s_{3}$, for $s_{2} \neq 0_{\mathbb{S}}$. Whenever clear from the context we will omit annotation $\mathbb{S}$ from operators.

Sets $\mathbb{B}$, with disjunction and conjunction, and $\mathbb{R}, \mathbb{N}$, with sum and products, are examples of (commutative) semi-rings. In $\mathbb{R}$, division is the inverse of product. In the sequel we let $\mathbb{C}, \mathbb{C}^{\prime}, \mathbb{C}_{1}, \ldots$ denote commutative semi-rings. For generic non-empty set $S$ and commutative semi-ring $\mathbb{C}$, we let $\mathbf{T F}(S, \mathbb{C})$ denote the set of total functions from $S$ to $\mathbb{C}$, and we let $\mathscr{P}, \mathscr{Q}, \mathscr{R}, \ldots$ range over it. We let $\mathbf{F T F}(S, \mathbb{C})$ denote the subset of $\mathbf{T F}(S, \mathbb{C})$ containing only those functions with finite support. $\mathscr{P}$ is an element of $\mathbf{F T F}(S, \mathbb{C})$ if and only if there 
exist $\left\{s_{1}, \ldots, s_{m}\right\} \subseteq S$, the support of $\mathscr{P}$, such that $\mathscr{P} s_{j} \neq 0_{\mathbb{C}}$ for $j=1 \ldots m$ and $\mathscr{P} s=0_{\mathbb{C}}$ for all $s \in S \backslash\left\{s_{1}, \ldots, s_{m}\right\}$. We equip $\mathbf{F T F}(S, \mathbb{C})$ with the operators defined below. The resulting algebraic structure of the set of finite support functions will be crucial for the compositional features of our approach.

Definition 2.4 Basic operators in $\mathbf{F T F}(S, \mathbb{C})$. If $s, s_{1}, \ldots, s_{m} \in S$, with $s_{i} \neq s_{j}$ for $i \neq j$, $\gamma_{1}, \ldots, \gamma_{m} \in \mathbb{C}, \mathscr{P}$ and $\mathscr{Q}$ in $\operatorname{FTF}(S, \mathbb{C})$, and $S^{\prime} \subseteq S$ then:

(1) $\left[s_{1} \mapsto \gamma_{1}, \ldots, s_{m} \mapsto \gamma_{m}\right]_{\mathbb{C}}$ denotes the following function:

$$
\left[s_{1} \mapsto \gamma_{1}, \ldots, s_{m} \mapsto \gamma_{m}\right]_{\mathbb{C}} s==_{\operatorname{def}} \begin{cases}\gamma_{j} & \text { if } s=s_{j} \in\left\{s_{1}, \ldots, s_{m}\right\}, \\ 0_{\mathbb{C}} & \text { otherwise. }\end{cases}
$$

the $0_{\mathbb{C}}$ constant function in $\operatorname{FTF}(S, \mathbb{C})$ is denoted by []

(2) function + is defined as $(\mathscr{P}+\mathscr{Q}) s==_{\mathrm{def}}(\mathscr{P} s)+_{\mathbb{C}}(\mathscr{Q} s)$;

(3) function $\boxplus$ is defined as $\boxplus \mathscr{P} S^{\prime}==_{\operatorname{def}} \sum_{\mathbb{C}_{s \in S^{\prime}}}(\mathscr{P} S)$ where $\sum_{\mathbb{C}}$ denotes the n-ary extension of $+_{\mathbb{C}}$. We let $\oplus \mathscr{P}$ be defined as $\oplus \mathscr{P}={ }_{\text {def }} \boxplus \mathscr{P} S$

Notice that all the above summations are over finite sets, due to the definition of $\operatorname{FTF}(S, \mathbb{C})$.

\subsection{State to Function Labelled Transition Systems}

In this section we introduce the notion of Simple State to Function Labelled Transition Systems, simple FuTSs for short, which is sufficient as underlying model for all fully Markovian SPCs presented in the paper. General FuTSs will be defined in Sect. 8.

Definition 2.5. A simple state to function A-labelled transition system (simple FuTS) over $\mathbb{C}$ is a tuple $(S, A, \mathbb{C}, \mapsto)$ where $S$ is a countable, non-empty, set of states, $A$ is a countable, non-empty, set of transition labels, $\mathbb{C}$ is a commutative semi-ring, and $\mapsto \subseteq$ $S \times A \times \mathbf{T F}(S, \mathbb{C})$ is the $A$-labelled transition relation.

As usual, we let $s \stackrel{\alpha}{\rightarrow} \mathscr{P}$ denote $(s, \alpha, \mathscr{P}) \in \nrightarrow$. Intuitively, $s_{1} \stackrel{\alpha}{\longmapsto} \mathscr{P}$ and $\left(\mathscr{P} s_{2}\right)=$ $\gamma \neq 0_{\mathbb{C}}$ means that $s_{2}$ is reachable from $s_{1}$ via (the execution of) $\alpha$ with a value $\gamma \in \mathbb{C}$. $\left(\mathscr{P} s_{2}\right)=0_{\mathbb{C}}$ means that $s_{2}$ is not reachable from $s_{1}$ via the above $\alpha$-transition; notice, however, that, unless the FuTS is deterministic (see Def. 2.6 below), there might be another $(\alpha-)$ transition from $s_{1}$ such that $s_{2}$ is reachable, via that transition. In the following, we will omit the adjective 'simple' when this will be clear from the context. Whenever necessary or convenient an initial state $s_{0} \in S$ will be identified, and the relevant FuTS will be the tuple $\left(S, A, \mathbb{C}, \neg, s_{0}\right)$. Henceforth, FuTSs will be denoted by $\mathcal{R}, \mathcal{R}_{1}, \mathcal{R}^{\prime}, \ldots$. Whenever $s \stackrel{\alpha}{\mapsto} \mathscr{P}$ is a transition of $\mathcal{R}$, we call $\mathscr{P}$ next state function, or continuation function, or, simply, continuation. In Sect. 8 the above definition will be extended to general FuTS, where different type of continuations are allowed within the same FuTS.

Definition 2.6. Let $\mathcal{R}=(S, A, \mathbb{C}, \mapsto)$ be a FuTS, then:

(1) $\mathcal{R}$ is total if for all $s \in S$ and $\alpha \in A$ there exists $\mathscr{P} \in \mathbf{T F}(S, \mathbb{C})$ such that $s \stackrel{\alpha}{\longmapsto} \mathscr{P}$;

(2) $\mathcal{R}$ is deterministic if for all $s \in S, \alpha \in A$, and $\mathscr{P}, \mathscr{Q} \in \mathbf{T F}(S, \mathbb{C})$ we have that the following holds: $s \stackrel{\alpha}{\longmapsto} \mathscr{P}, s \stackrel{\alpha}{\rightarrow} \mathscr{Q} \Longrightarrow \mathscr{P}=\mathscr{Q}$;

(3) $\mathcal{R}$ is a finite support FuTS (FsFuTS for short) if $\neg \subseteq S \times A \times \mathbf{F T F}(S, \mathbb{C})$. 
Henceforth, we will consider only FsFuTSs, since they are powerful enough to model the process calculi we are interested in. Notice that considering deterministic FUTS does not imply that they can only be used to model deterministic behaviors (see Sect. 8.1).

In the context of Markovian SPCs, where the relevant commutative semi-ring $\mathbb{C}^{\text {is }} \mathbb{R}_{\geq 0}$, $\gamma$ characterizes either the duration of an action $a$, denoted by $\delta_{a}$ in this paper, or just the passage of time, denoted by $\delta$ in this paper. In any case, such time interval is modeled as an exponentially distributed $\mathrm{RV}$, and $\gamma>0$ is the relevant rate. In the sequel we use $\delta^{e}$ in place of $\delta$, and $\delta_{a}^{e}$ in place of $\delta_{a}$, to emphasize the exponential nature of random delays.

Definition 2.7. Given set $\mathcal{A}$ of actions, the set of action delays $\Delta_{\mathcal{A}}$ is $\left\{\delta_{a}^{e} \mid a \in \mathcal{A}\right\}$.

Remark 2.8. It is easy to see that standard CTMCs are the same as total deterministic $\left\{\delta^{e}\right\}$-labeled FsFuTS over $\mathbb{R}_{\geq 0}$. Similarly, by using the transition label $\pi$ for denoting discrete random experiments, DTMCs are the same as total deterministic $\{\pi\}$-labeled FsFUTS over $\mathbb{R}_{\geq 0}$, with the additional requirement that every continuation $\mathscr{P}$ is a probability distribution function, i.e. $\oplus \mathscr{P}=1$; note that $\oplus \mathscr{P}=1$ implies $\mathscr{P} s \in[0,1]$ since the range of $\mathscr{P}$ contains only non-negative values. RTSs coincide with $\Delta_{\mathcal{A}}$-labeled FsFUTS over $\mathbb{R}_{\geq 0}$. Finally, given a generic set of weights $K$, let $\mathbb{K}$ be a commutative semi-ring such that $K \subset \mathbb{K}$ and $0_{\mathbb{K}} \notin K$; let, furthermore, $\omega$ label weighted transitions; then Weighted Transition Systems (WTSs), as defined in [Fahrenberg et al. 2011], are total $\{\omega\}$-labeled FUTS over $\mathbb{K}$, with the additional property that for each $s \in S$ and $\mathscr{P} \in \mathbf{T F}(S, \mathbb{K})$ such that $s \stackrel{\omega}{\longmapsto} \mathscr{P}$, there exists an $s^{\prime} \in S$ such that $\mathscr{P} s^{\prime} \neq 0_{\mathbb{K}}$; this last property, together with totality, corresponds, in the FUTS framework, to the non-blocking property required in the definition of WTSs.

The definition of bisimilarity over FUTS is reported below.

Definition 2.9. Given a FuTS $(S, A, \mathbb{C}, \mapsto)$, we say that an equivalence relation $B \subseteq$ $S \times S$ is a bisimulation relation if and only if, whenever $\left(s_{1}, s_{2}\right) \in B$, for all $\alpha \in A$ and $\mathscr{P}_{1}$, if $s_{1} \stackrel{\alpha}{\mapsto} \mathscr{P}_{1}$, then there exists $\mathscr{P}_{2}$ such that $s_{2} \stackrel{\alpha}{\mapsto} \mathscr{P}_{2}$ and $\boxplus \mathscr{P}_{1} C=\boxplus \mathscr{P}_{2} C$ for all equivalence classes $C \subseteq S$ of $B$. We say that $s_{1}$ and $s_{2}$ are bisimilar, written $s_{1} \sim s_{2}$, if and only if $\left(s_{1}, s_{2}\right) \in B$ for some bisimulation relation $B$.

Definition 2.10. For FsFuTS $\mathcal{R}=(S, A, \mathbb{C}, \neg), s \in S$, and $\Gamma \subseteq A$, the set of states reachable from $s$ via $\Gamma$, denoted $S /_{s, \Gamma}$, is the least set such that $s \in S /_{s, \Gamma}$ and if $s^{\prime} \in S /_{s, \Gamma}$, $s^{\prime} \stackrel{\alpha}{\longmapsto} \mathscr{P}$ for some $\alpha \in \Gamma$ and $\mathscr{P} \in \mathbf{F T F}(S, \mathbb{C})$ with $\mathscr{P} s^{\prime \prime} \neq 0_{\mathbb{C}}$, then $s^{\prime \prime} \in S /_{s, \Gamma \text {. Notice }}$

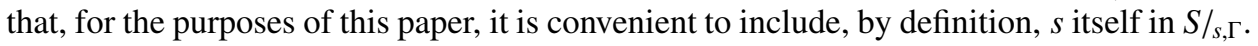
We furthermore define the set of actions associated with the set of states reachable from $s$ via $\Gamma, A / s, \Gamma \subseteq A$, as follows:

$$
A / s, \Gamma={ }_{\operatorname{def}}\left\{\alpha \in A \mid \exists s^{\prime} \in S /_{s, \Gamma}, \mathscr{P} \in \mathbf{F T F}(S, \mathbb{C}) . s^{\prime} \stackrel{\alpha}{\longmapsto} \mathscr{P} \text { and } \mathscr{P} \neq[]_{\mathbb{C}}\right\}
$$

and, for $\mathscr{P} \in \mathbf{F T F}(S, \mathbb{C})$, we let $\left.\mathscr{P}\right|_{s, \Gamma} \in \mathbf{F T F}\left(S /_{s, \Gamma}, \mathbb{C}\right)$ denote $\mathscr{P}_{\mid\left(\left.S\right|_{s, \Gamma}\right)}$, i.e. the restriction

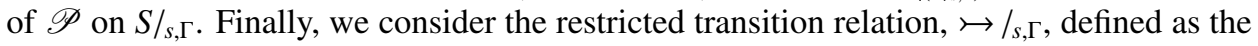
set $\left\{\left(s^{\prime}, \alpha, \mathscr{P} / s, \Gamma\right) \mid s^{\prime} \in S / s, \Gamma, \alpha \in A / s, \Gamma, s^{\prime} \stackrel{\alpha}{\rightarrow} \mathscr{P}\right\}$

The FsFuTS generated from a state $s$ is defined below:

Definition 2.11. Let $\mathcal{R}=(S, A, \mathbb{C}, \neg)$ be a FsFuTS, $s \in S$, and $\Gamma \subseteq A$. The FsFuTS generated from $s$ and $\Gamma$, denoted by $\mathcal{R} / s, \Gamma$, is the FsFuTS $\left(S /_{s, \Gamma}, A /_{s, \Gamma}, \mathbb{C}, \longmapsto /_{s, \Gamma}, s\right)$. 


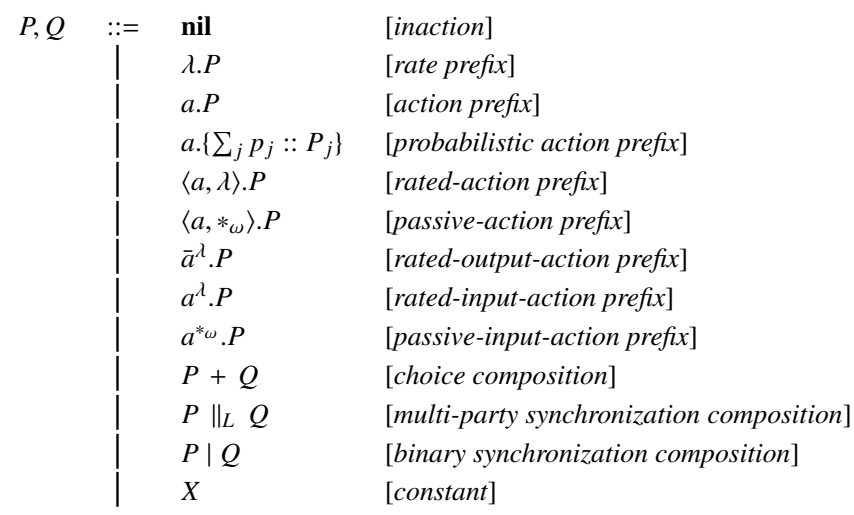

Fig. 2. Syntax of Stochastic Process Calculi operators.

For the sake of notational clarity, whenever $\Gamma=A$, we write $\mathcal{R} / s\left(S / s, \mapsto /_{s}\right.$, respectively) in place of $\mathcal{R} / s, \Gamma\left(S /_{s, \Gamma}, \mapsto /_{s, \Gamma}\right.$, respectively).

\section{OPERATORS OF STOCHASTIC PROCESSES}

In this paper we consider several SPCs proposed in the literature. For reasons of notational uniformity, we refer to the grammar of Fig. 2. Whenever we deal with a specific SPC, we select those constructs which are relevant for that language. As a consequence, we often use a concrete syntax which is different from that originally defined for the specific SPC. For the sake of completeness, in Appendix A, the main differences between the syntax we use and the original ones will be pointed out. In the grammar of Fig. 2 we assume that rates $\lambda, \lambda^{\prime}, \lambda_{1}, \ldots$ and weights $\omega, \omega^{\prime}, \omega_{1}, \ldots$ range over $\mathbb{R}_{>0}$; we furthermore assume non-empty, countable sets $\mathcal{A}$ of actions, ranged over by $a, a^{\prime}, a_{1}, \ldots$, and $\overline{\mathcal{A}}$ of co-actions, ranged over by $\bar{a}, \bar{a}^{\prime}, \bar{a}_{1}, \ldots$ Finally, $L \subset\left(\wp_{f i n} \mathcal{A}\right)$ is a finite set of actions.

Term nil denotes the process that is unable to get involved in any action. The syntax contains many types of prefixes needed to address different types of calculi. Rate prefix $\lambda . P$, delays execution of $P$ by an interval, conventionally denoted by $\delta^{e}$, the duration of which is an exponentially distributed RV with rate $\lambda \in \mathbb{R}_{>0}$. Action prefix a.P starts with the execution of action $a$ and then continues with that of $P$; the execution of $a$ is duration-less or instantaneous, i.e. takes no time. Probabilistic action prefix a. $\left\{p_{1}:: P_{1}+\ldots+p_{h}:: P_{h}\right\}$ generalizes action prefix in that the single process $P$ is replaced by a probability subdistribution over processes, where $p_{j}>0$ for $0<j \leq h$ and $\sum_{j=1}^{h} \leq 1$ is required, as well as $P_{i} \neq P_{j}$ for $i \neq j$. The intended meaning is that $a .\left\{p_{1}:: P_{1}+\ldots+p_{h}:: P_{h}\right\}$ starts with the execution of action $a$ and then may continue with one of the processes $P_{1}, \ldots, P_{h}$, the probability of continuing with $P_{j}$ being $p_{j}$.

In rated-action prefix $\langle a, \lambda\rangle . P$ the duration of the execution of action $a$ is an exponentially distributed RV with rate $\lambda$; after completion of the execution of $a$, the behavior continues as in $P$. Rated-action prefix is typical of most SPCs which have been proposed in the literature and which are based on the multi-party synchronization paradigm. In such languages there is often the possibility of leaving the durations of some of the action 'unspecified', in the sense that, for any such 'passive action', the actual duration is determined by the rate of the 'active' action with which it synchronizes. The passive-action prefix 
operator $\left\langle a, *_{\omega}\right\rangle . P$ serves this purpose; weight $\omega$ is used for determining a probabilistic distribution in case there is more than one passive action which may synchronize with the same active one.

Rated-input-action prefix $a^{\lambda} . P$, and rated-output-action prefix $\bar{a}^{\lambda} . P$ are used to model one-to-one synchronization paradigms; rates are associated to both input and output actions. There are also proposals in which input actions are considered passive by definition, thence the passive-input-action prefix $a^{*} \omega . P$. In all cases above, after executing $a$, the process continues as $P$.

The choice composition operator is denoted by $P_{1}+P_{2}$. In fully Markovian calculi the term $P_{1}+P_{2}$ is interpreted according to the race condition principle of CTMCs. For instance, the sojourn time in state $\langle a, \lambda\rangle$.nil $+\langle b, \mu\rangle$.nil is an exponentially distributed RV with rate $\lambda+\mu$. There is a race between the execution of action $a$ and action $b$. The probability that the race is 'won' by $a(b)$ is $\frac{\lambda}{\lambda+\mu}\left(\frac{\mu}{\lambda+\mu}\right)$. In calculi like IML, instead, $P_{1}+P_{2}$ can model a race condition (e.g. by $\mu_{1} \cdot Q_{1}+\mu_{2} \cdot Q_{2}$ ), or a standard non-deterministic choice (e.g. by $a_{1} \cdot Q_{1}+a_{2} \cdot Q_{2}$ ), or an arbitrary mix thereof. Similar considerations apply to the Language for Markov Automata.

The multi-party synchronization parallel composition operator, denoted by $P_{1} \|_{L} P_{2}$, where $L \in\left(\wp_{f i n} \mathcal{A}\right)$ is the synchronization (or cooperation) set, prescribes that actions in $L$ be performed synchronously by both $P_{1}$ and $P_{2}$, while the other actions be performed independently by the two processes. The one-to-one synchronization parallel composition, denoted by $P_{1} \mid P_{2}$, is used to model synchronization of complementary actions. Finally, $X$ is a constant defined by means of an equation of the form $X:=P$ where constants $X, X_{1}, X^{\prime}, \ldots$ may occur only guarded in $P$, i.e. within the scope of a prefix ..Q. A set $E$ of defining equations is complete and consistent if and only if it contains exactly one defining equation for each constant of the language. For all practical purposes, it is sufficient to consider only finite (and consistent) sets of defining equations $\left\{X_{1}:=P_{1}, \ldots X_{n}:=P_{n}\right\}$ which can easily be completed by adding equation $X:=$ nil for all $X \notin\left\{X_{1}, \ldots X_{n}\right\}$. In this paper, the FuTS semantics of a calculus $C$, will be given up to a complete and consistent set of constant defining equations $E$. In particular, a FuTS $\mathcal{R}_{C}$ will be defined, corresponding to the complete language of the calculus, under $E$. The set of states of the FuTS coincides with the set of terms $\mathcal{P}_{C}$ of the calculus; the label set $\mathcal{L}_{C}$ typically (but not necessarily) refers to sets $\mathcal{A}$ and $\overline{\mathcal{A}}$ of actions and co-actions; the transition relation is defined by means of a logical deduction system and depends on the equations in $E$. The FuTS of $a$ single process $P \in \mathcal{P}_{C}$ is, as usual, defined as the FuTS generated by $P$, i.e. $\mathcal{R}_{C} / P$. Notice that, in the following sections, in the definition of the FuTS semantics of each calculus, set $E$ will not be mentioned explicitly.

\section{A LANGUAGE FOR CTMCs}

In this section we define a simple language for describing CTMCs like in [Hermanns et al. 2002]. The set $\mathcal{P}_{C T M C}$ of CTMC terms is defined by the grammar obtained by selecting from Fig. 2 the following operators: inaction, rate prefix, choice composition, and constant.

In the sequel we focus on the semantics. The relevant set of states is $\mathcal{P}_{C T M C}$. For what concerns the set of labels, we first observe that standard CTMCs are composed of states, transitions between states, and rates characterizing transition delays. In particular, standard CTMCs abstract from specific actions. We denote the (action-less) passage of time with $\delta^{e}$. Consequently, the label set $\mathcal{L}_{C T M C}$ is the singleton $\left\{\delta^{e}\right\}$ and the relevant semi-ring is 


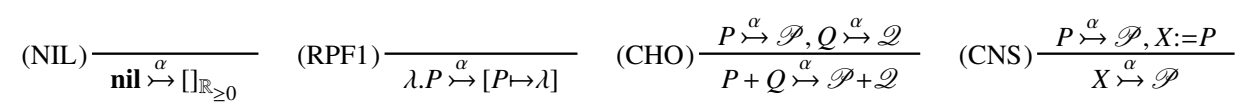

Fig. 3. Semantics Rules for the Language of CTMCs.

$\mathbb{R}_{\geq 0}$. The transition relation $\nrightarrow$ is the one defined in Figure 3, where $\alpha=\delta^{e}$. Notice that the third component of the transition relation is no longer a state, as in traditional SOS, but a continuation function, which maps states to rates. Before giving the formal definition of the FuTS semantics of the language, we briefly discuss the intuitions behind the rules in Fig. 3. Intuitively, it should be clear from Rule (NIL) that no state is reachable from nil. According to Rule (RPF1), $P$ is reachable from $\lambda . P$ and the rate of such a transition is $\lambda$.

The rule for choice (CHO) prescribes that $P+Q$ reaches a state $R$ with a rate resulting from the sum of the rates by which the individual components may reach $R$. If one of the components, say $Q$, cannot reach $R$, then $(\mathscr{Q} R)=0$ and only the contribution of the other is considered. It is worth noting that the continuation $\mathscr{P}+\mathscr{Q}$ of $P+Q$ after $\delta^{e}$ is defined compositionally from the continuations $\mathscr{P}$ and $\mathscr{Q}$ of $P$ and $Q$. Furthermore, such a continuation is defined by means of just one rule, while, typically, choice operator semantics definitions require two rules. The use of FuTSs, in particular in the rule for choice, naturally handles race conditions and solves the related transition multiplicity issue in a simple and elegant way. In Fig. 4(a) the possible transitions associated with $\lambda \cdot R_{1}+$ $\mu . R_{2}$ are presented where continuation functions associating rates to future behaviors are represented as dotted arrows. In this simple example, the following transitions can be derived:

(1) if $R_{1} \neq R_{2}$ then $\lambda . R_{1}+\mu . R_{2} \stackrel{\delta^{e}}{\rightarrow}\left[R_{1} \mapsto \lambda, R_{2} \mapsto \mu\right]$;

(2) if $R_{1}=R_{2}$ then $\lambda . R+\mu . R \stackrel{\delta^{e}}{\mapsto}[R \mapsto \lambda+\mu]$

(3) if $R_{1}=R_{2}$ and $\lambda=\mu$ then $\lambda . R+\lambda . R \stackrel{\delta^{e}}{\mapsto}[R \mapsto 2 \lambda]$.

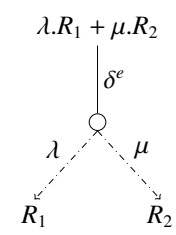

(1)

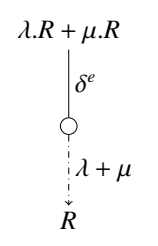

(2)

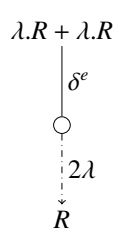

(3)

(a) FuTS and race condition.

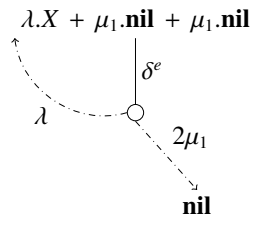

(b) A CTMC

Fig. 4. Examples of FuTS representations of CTMCs

Rule (CNS) for process constants should be obvious. The presence of $X:=P$ in the premises for the rule for constant definition is intended as the fact that $X:=P$ is an element of the relevant set $E$ of defining equations and $X$ behaves exactly like $P$. The CTMC associated with term $X$, when $X:=\lambda . X+\mu_{1}$.nil $+\mu_{1}$.nil, is the one of Fig. 4(b).

The following proposition guarantees that the continuation functions of interest are finite-support and total.

ACM Computing Surveys - In Press, Vol. , No., November 2012. 
Proposition 4.1. For all $P \in \mathcal{P}_{C T M C}$ and $\mathscr{P} \in \mathbf{T F}\left(\mathcal{P}_{C T M C}, \mathbb{R}_{\geq 0}\right)$, if $P \stackrel{\delta^{e}}{\rightarrow} \mathscr{P}$ can be derived using the rules of Fig. 3, then $\mathscr{P} \in \mathbf{F T F}\left(\mathscr{P}_{C T M C}, \mathbb{R}_{\geq 0}\right)$.

We are now ready for providing the formal definition of the FUTS semantics for the language of CTMCs:

Definition 4.2. The formal semantics of the calculus for CTMCs is the FsFuTS $\mathcal{R}_{C T M C}$ $\left(\mathcal{P}_{C T M C}, \mathcal{L}_{C T M C}, \mathbb{R}_{\geq 0}, \nrightarrow\right)$; where $\leadsto \subseteq \mathcal{P}_{C T M C} \times \mathcal{L}_{C T M C} \times \mathbf{F T F}\left(\mathcal{P}_{C T M C}, \mathbb{R}_{\geq 0}\right)$ is the least relation satisfying the rules of Fig. 3 .

The following theorem characterizes the structure of $\mathcal{R}_{C T M C}$ :

Theorem 4.3. $\mathcal{R}_{\text {CTMC }}$ is total and deterministic.

As a corollary of Theorem 4.3 we obtain that whenever $P \stackrel{\delta^{e}}{\longmapsto} \mathscr{P}$, the exit rate of $P$ is given by $\oplus \mathscr{P}$ and $\mathscr{P}$ is the row of the rate matrix corresponding to $P$.

\section{PARALLEL COMPOSITION}

In this section we introduce parallel composition and we show the basic principles for handling it in the FuTS framework. Here we focus on a pure interleaving composition operator, incorporating it in the simple language of CTMCs which has been presented in Sect. 4. In doing so, we will introduce some additional notation useful in the remaining sections, where major SPCs will be presented and their specific design choices will be addressed.

\subsection{Parallel Composition of CTMCs}

Let us consider processes $\lambda_{1} \cdot P_{1}$ and $\lambda_{2} . P_{2}$, for some $P_{1}$ and $P_{2}$; we are interested in their (interleaving) parallel composition, i.e. $\lambda_{1} \cdot P_{1} \| \lambda_{2} \cdot P_{2}$. The interleaving assumption prescribes that any state reachable in one step from $\lambda_{1} \cdot P_{1} \| \lambda_{2} \cdot P_{2}$ must be a term of the form $Q_{1} \| Q_{2}$. To be more precise, the only terms reachable via a (single) step from term $\lambda_{1} \cdot P_{1} \| \lambda_{2} \cdot P_{2}$ are $P_{1} \| \lambda_{2} \cdot P_{2}$, reachable with a random delay which is exponentially distributed, with rate $\lambda_{1}$, and $\lambda_{1} . P_{1} \| P_{2}$, reachable with a similar delay, but with rate $\lambda_{2}$. Intuitively, we can see that the parallel composition of $\lambda_{1} \cdot P_{1}$ and $\lambda_{2} . P_{2}$ results into a CTMC where the exit rate of state $\lambda_{1} \cdot P_{1} \| \lambda_{2} \cdot P_{2}$ is $\lambda_{1}+\lambda_{2}$ and there are two transitions as above. Notice that if $\lambda_{1}=\lambda_{2}=\lambda$ and $P_{1}=P_{2}=P$, the exit rate of $\lambda . P \| \lambda . P$ must be $2 \cdot \lambda$, due to the race condition principle. Finally, assuming for simplicity $P_{1}=P_{2}=$ nil, and recalling the basic memoryless property of negative exponential distributions, we note that the time needed for reaching state nil $\|$ nil from state $\lambda_{1}$.nil $\| \lambda_{2}$.nil, is a $\operatorname{RV} D=\operatorname{MAx}\left\{D_{1}, D_{2}\right\}$ where $D_{j}$ is an exponentially distributed $\mathrm{RV}^{3}$ with rate $\lambda_{j}$.

In the sequel, we will see how the notions discussed above can be expressed in terms of continuation functions, so that we can extend the semantics of the language of CTMCs in order to include the interleaving parallel composition operator. Let $P_{1}$ and $P_{2}$ be generic terms of our CTMC language, with $P_{1} \stackrel{\delta^{e}}{\longmapsto} \mathscr{P}_{1}$ and $P_{2} \stackrel{\delta^{e}}{\longmapsto} \mathscr{P}_{2}$ and let us consider $\mathscr{P}$ such that $P_{1} \| P_{2} \stackrel{\delta^{e}}{\hookrightarrow} \mathscr{P}$. We know that for all terms $Q$ which are not of the form $Q_{1} \| Q_{2}$, for

${ }^{3}$ We remind the reader that $D$ is not an exponentially distributed RV. Technically, $D$ belongs to the class of Phase Type distributions which are combinations of exponentially distributed RVs and can profitably be represented as CTMCs with an absorbing state (nil || nil, in our example). The interested reader is referred to [Neuts 1981]. 
some $Q_{1}$ and $Q_{2}, \mathscr{P} Q=0$ must hold. Furthermore, $Q_{1} \| Q_{2}$ is reachable from $P_{1} \| P_{2}$ if and only if $Q_{1}$ is reachable from $P_{1}$ and $Q_{2}$ is the term $P_{2}$ or, symmetrically, $Q_{2}$ is reachable from $P_{2}$ and $Q_{1}$ is the term $P_{1}$. As far as rates are concerned, $Q_{1} \| P_{2}$ would be reachable with rate $\mathscr{P}_{1} Q_{1}$, i.e. $\mathscr{P}\left(Q_{1} \| P_{2}\right)=\mathscr{P}_{1} Q_{1}$, while $P_{1} \| Q_{2}$ would be reachable with rate $\mathscr{P}_{2} Q_{2}$, i.e. $\mathscr{P}\left(P_{1} \| Q_{2}\right)=\mathscr{P}_{2} Q_{2}$, keeping in mind that any term $Q$ reachable both via a step of $P_{1}$ and via a step of $P_{2}$ is reachable with the cumulative rate $\mathscr{P}_{1} Q+\mathscr{P}_{2} Q$. In the sequel, we show how continuation $\mathscr{P}$ can be obtained as an appropriate composition of the component continuations $\mathscr{P}_{1}$ and $\mathscr{P}_{2}$. This will enable us to define the semantics of the interleaving, and in general the parallel composition operator, in a compositional way.

In the following, we assume an extended definition of set $\boldsymbol{P}_{\text {CTMC }}$, which includes also interleaving terms of the form $P_{1} \| P_{2}$. We first of all 'lift' the interleaving operator $\|$ to a product operator $\otimes_{\|}$on continuation functions as follows:

$$
\left(\mathscr{P}_{1} \otimes_{\|} \mathscr{P}_{2}\right) Q==_{\text {def }}\left\{\begin{array}{l}
\left(\mathscr{P}_{1} Q_{1}\right) \cdot\left(\mathscr{P}_{2} Q_{2}\right), \text { if } Q=Q_{1} \| Q_{2} \text { for some } Q_{1}, Q_{2} \in \mathcal{P}_{C T M C}, \\
0, \text { otherwise. }
\end{array}\right.
$$

Furthermore, for each term $P \in \mathcal{P}_{C T M C}$ we let the characteristic function $\mathcal{X}: \mathcal{P}_{C T M C} \rightarrow$ $\mathbb{R}_{\geq 0}$ be defined as $\left[P \mapsto 1_{\mathbb{R}_{\geq 0}}\right.$, yielding 1 on $P$ and 0 on any other term. Continuation $\mathscr{P}$ above can easily be expressed as a composition of continuations $\mathscr{P}_{1}$ and $\mathscr{P}_{2}$ and the component terms $P_{1}$ and $P_{2}$, i.e. $\mathscr{P}=\mathscr{P}_{1} \otimes_{\|}\left(X P_{2}\right)+\left(X P_{1}\right) \otimes_{\|} \mathscr{P}_{2}$.

The actual rule for the interleaving operator of CTMCs is the following:

$$
\text { (INT) } \frac{P_{1} \stackrel{\delta^{e}}{\rightarrow} \mathscr{P}_{1}, P_{2} \stackrel{\delta^{e}}{\rightarrow} \mathscr{P}_{2}}{P_{1} \| P_{2} \stackrel{\delta^{e}}{\rightarrow}\left(\mathscr{P}_{1} \otimes_{\|}\left(X P_{2}\right)\right)+\left(\left(X P_{1}\right) \otimes_{\|} \mathscr{P}_{2}\right)}
$$

For instance, for term $\lambda_{1}$.nil $\| \lambda_{2}$.nil, we have the transitions $\lambda_{1}$.nil $\stackrel{\delta^{e}}{\rightarrow}\left[\right.$ nil $\mapsto \lambda_{1}$ ] and $\lambda_{2}$.nil $\stackrel{\delta^{e}}{\mapsto}\left[\right.$ nil $\left.\mapsto \lambda_{2}\right]$. By applying rule (INT) above, we obtain:

$$
\frac{\lambda_{1} \text {.nil } \stackrel{\delta^{e}}{\mapsto}\left[\text { nil } \mapsto \lambda_{1}\right], \lambda_{2} \text {.nil } \stackrel{\delta^{e}}{\mapsto}\left[\text { nil } \mapsto \lambda_{2}\right]}{\lambda_{1} \text {.nil } \| \lambda_{2} \text {.nil } \stackrel{\delta^{e}}{\rightarrow}\left[\text { nil } \mapsto \lambda_{1}\right] \otimes_{\|}\left(X \lambda_{2} \text {.nil }\right)+\left(X \lambda_{1} \text {.nil }\right) \otimes_{\|}\left[\text {nil } \mapsto \lambda_{2}\right]}
$$

where:

$$
\begin{aligned}
& {\left[\text { nil } \mapsto \lambda_{1}\right] \otimes_{\|}\left(\mathcal{X} \lambda_{2} \text {.nil }\right)+\left(\mathcal{X} \lambda_{1} \text {.nil }\right) \otimes_{\|}\left[\text {nil } \mapsto \lambda_{2}\right] } \\
= & {\left[\text { nil } \mapsto \lambda_{1}\right] \otimes_{\|}\left[\lambda_{2} \text {.nil } \mapsto 1\right]+\left[\lambda_{1} \text {.nil } \mapsto 1\right] \otimes_{\|}\left[\text {nil } \mapsto \lambda_{2}\right] } \\
= & {\left[\text { nil } \| \lambda_{2} \text {.nil } \mapsto \lambda_{1}\right]+\left[\lambda_{1} \text {.nil } \| \text { nil } \mapsto \lambda_{2}\right] } \\
= & {\left[\text { nil } \| \lambda_{2} \text {.nil } \mapsto \lambda_{1}, \lambda_{1} \text {.nil } \| \text { nil } \mapsto \lambda_{2}\right] }
\end{aligned}
$$

stating that term $\lambda_{1}$.nil $\| \lambda_{2}$.nil can reach nil $\| \lambda_{2}$.nil with rate $\lambda_{1}$ and $\lambda_{1}$.nil $\|$ nil with rate $\lambda_{2}$. The FuTS generated by $\lambda_{1}$.nil $\| \lambda_{2}$.nil is reported in Fig. 5 (a). Let us now consider the term $X \| X$, where $X:=\lambda . X$, that represents a subtle situation where it is crucial to take into account all possible derivations and consider their cumulative effect. The FuTS associated to $X \| X$ is shown in Fig. 5 (b). We have that $X \stackrel{\delta^{e}}{\mapsto}[X \mapsto \lambda]$ and, by applying rule (INT):

$$
\frac{X \stackrel{\delta^{e}}{\mapsto}[X \mapsto \lambda], X \stackrel{\delta^{e}}{\rightarrow}[X \mapsto \lambda]}{X \| X \stackrel{\delta^{e}}{\mapsto}[X \mapsto \lambda] \otimes_{\|}(X X)+(X X) \otimes_{\|}[X \mapsto \lambda]}
$$

and, with a derivation similar to the above one, we obtain $X \| X \stackrel{\delta^{e}}{\mapsto}[X \| X \mapsto 2 \cdot \lambda]$. Thus, while according to the standard semantics of process algebras $X \| X$ would be considered equivalent to $X$, in SPCs $X \| X$ is modeled as twice as fast as $X$. The use of summation 

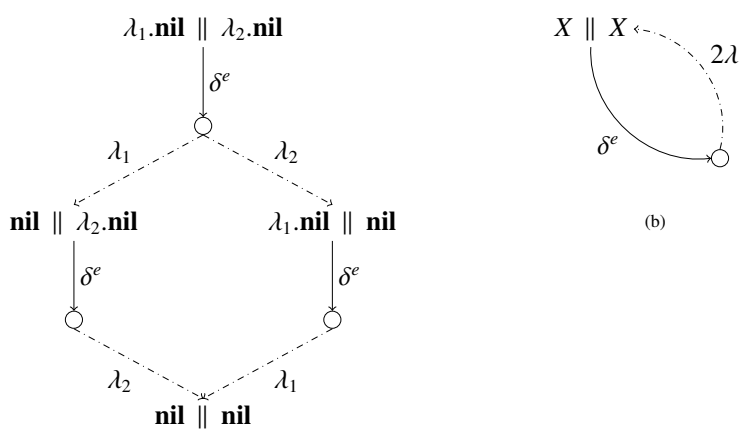

(b)

(a)

Fig. 5. Parallel composition of CTMCs.

to compose the next state functions associated with each of the two parallel components, guarantees the correct calculation of the rates of all transitions.

\subsection{Generalizing auxiliary operators}

In this section we generalize the operators on continuation functions introduced for the language of CTMCs in order to use them for other calculi and for richer interpretations. In the following we assume set $S$ and semi-ring $\mathbb{C}$ as in Sect. 2.

Characteristic functions. The $(S, \mathbb{C})$-characteristic function $\mathcal{X}_{\mathbb{C}}: S \rightarrow \operatorname{FTF}(S, \mathbb{C})$ is defined by: $\chi_{\mathbb{C}} s={ }_{\text {def }}\left[s \mapsto 1_{\mathbb{C}}\right]$ and is the function which yields $1_{\mathbb{C}}$ on $s$ and $0_{\mathbb{C}}$ elsewhere. Also for $\mathcal{X}_{\mathbb{C}}$, we will omit the subscript $\mathbb{C}$ whenever clear from the context.

Parallel aggregation. Let $\times: S \rightarrow S \rightarrow S$ be an injective binary function on $S$. The parallel aggregation operator $\otimes_{\times}^{\mathbb{C}}: \mathbf{T F}(S, \mathbb{C}) \rightarrow \mathbf{T F}(S, \mathbb{C}) \rightarrow \mathbf{T F}(S, \mathbb{C})$ is defined below:

$$
\left(\mathscr{P}_{1} \otimes_{\times}^{\mathbb{C}} \mathscr{P}_{2}\right) s=_{\text {def }}\left\{\begin{array}{l}
\left(\mathscr{P}_{1} s_{1}\right) \cdot \mathbb{C}\left(\mathscr{P}_{2} s_{2}\right), \text { if } \exists s_{1}, s_{2} \in S . s=s_{1} \times s_{2}, \\
0_{\mathbb{C}}, \text { otherwise. }
\end{array}\right.
$$

Notice that injectivity of $\times$ is essential for the above definition to make sense; for each SPC we will consider, operator $x$ will be replaced by the parallel composition constructor specific of the calculus; thus $\otimes_{\times}^{\mathbb{C}}$ is parametric with respect to such specific operators; for instance, in the sequel we will use $\otimes_{\|_{L}}^{\mathbb{C}}$ and $\otimes_{\mid}^{\mathbb{C}}$ when multi-parties ( $\left.\|_{L}\right)$ and one-to-one composition $(\mid)$ are used, respectively.

Renormalization. Parallel aggregation combines functions to describe behavior of cooperating processes. However, to compute the rate associated with specific transitions, the aggregated values might have to be renormalized. Let $\mathscr{P}$ be a function in $\operatorname{TF}(S, \mathbb{C})$ and $x, y \in \mathbb{C}$; we let $\mathscr{P} \cdot \frac{x}{y}$ denote the function associating to each $s \in S(\mathscr{P} s) \cdot \mathbb{C} \frac{x}{y}$ when $y \neq 0_{\mathbb{C}}$ and $0_{\mathbb{C}}$ otherwise.

Parallel aggregation $\otimes_{\times}^{\mathbb{C}}$ binds stronger that multiplication and, consequently, renormalization. The following proposition follows directly from the definitions above:

Proposition 5.1. For every countable non empty set $S$ and commutative semi-ring $\mathbb{C}$, $\operatorname{FTF}(S, \mathbb{C})$ is closed under the operations $\mathcal{X}_{\mathbb{C}},\left(\otimes_{-} \otimes_{\times}{ }_{-}\right)$, and $-_{-} \mathbb{C}_{-} /_{-}$, i.e. for each $s \in S$, 
$\mathscr{P}, \mathscr{Q} \in \mathbf{F T F}(S, \mathbb{C})$, and $x, y \in \mathbb{C}$, we have that: $\left(\mathcal{X}_{\mathbb{C}} s\right),\left(\mathscr{P} \otimes_{\times} \mathscr{Q}\right)$ and $\mathscr{P} \cdot \mathbb{C} x / y$ are elements of $\mathbf{F T F}(S, \mathbb{C})$.

\section{FULLY MARKOVIAN CALCULI WITH MULTI-PARTY SYNCHRONIZATION}

A key issue which needs to be addressed when dealing with action based PCs is action synchronization. This involves fundamental design decisions on the nature of synchronization and of synchronization actions, namely those actions resulting from synchronizing actions. In the case of SPCs, the additional issue of the relationship between the stochastic-time features of synchronizing actions and those of synchronization actions must be addressed. The intuition behind the rendez vous nature of synchronization could naturally bring to require that the duration of a synchronization action should be the MAx of the durations of the synchronizing actions. Unfortunately, this cannot be achieved if one wants action durations be modeled by RVs with negative exponential distributions. We recall, in fact, that such a class of RVs is not closed under MAX. Consequently, in the literature several different conceptual frameworks have been proposed. One approach is to abandon the framework of exponential distributions and use general distributions; the problem here is that most of the theory and tools for the analysis of stochastic systems are based on exponential distributions. Another approach has been to de-couple actions from delays, and this will be dealt with in Sect. 8.1. In this section we focus on SPCs with multi-party synchronization which lay in the realm of exponential distributions: the duration of a synchronization action is assumed to be a RV with a negative exponential distribution, the rate of which is a (simple) function of the rates of the synchronizing actions. Furthermore, such functions are chosen in such a way that desirable properties like composition commutativity or associativity, are preserved. We will consider TIPP [Hermanns et al. 1998], EMPA [Aldini et al. 2010], and PEPA [Hillston 1996], among the major approaches addressing the issue of synchronization rates. In TIPP it is assumed that cooperating activities can boost each other, thus the synchronization rate is obtained as the product of those of the synchronizing actions. In EMPA, instead, a distinction is introduced between active and passive actions. A synchronization may take place only between a single active action and one or more passive actions. The synchronization rate is that of the active action. In PEPA, the synchronization rate of (active) actions is the minimum of the rates of the synchronizing components according to the intuition that the rate of the synchronization is equal to that of the slowest synchronizing action-this action has the strongest impact on the cooperation between the synchronizing actions. PEPA provides also the notion of passive, which do not affect the synchronization rate. We address only those fragments of the above mentioned SPCs which are relevant for stochastic behavior, ignoring operators like relabeling or hiding. We will call such fragments $\mathrm{TIPP}_{k}, \mathrm{EMPA}_{k}$, and $\mathrm{PEPA}_{k}$, the subscript $k$ standing for kernel.

\section{1 $\mathrm{TIPP}_{k}$}

The kernel we consider refers to the version of TIPP presented in [Hermanns et al. 1998] ${ }^{4}$. The considered operators are: inaction, rated-action prefix, choice, multi-party synchronization, and constant. The set $\mathcal{P}_{T_{I P P_{k}}}$ of $\mathrm{TIPP}_{k}$ terms is defined by the grammar obtained by selecting from Fig. 2 the specific productions for the above operators.

${ }^{4}$ In [Buchholz 1994; Hermanns et al. 1998], synchronization rates are defined as the product of those of the synchronizing actions, as opposed to the original definition of TIPP, given in [Gotz et al. 1993], where, instead, such rates are the MAX of the rates of the components.

ACM Computing Surveys - In Press, Vol. , No., November 2012. 

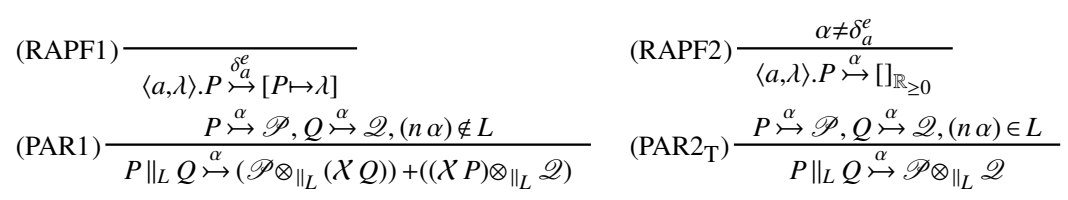

Fig. 6. Additional semantics rules for $\mathrm{TIPP}_{k}$

Let us now consider the ingredients for the definition of the FUTS semantics of TIPP ${ }_{k}$. $\mathcal{P}_{\text {TIPP }}$ is the relevant set of states, as usual. In TIPP, time delays are not separated from actions; consequently, $\mathcal{L}_{T I P P_{k}}$ is the set $\Delta_{\mathcal{A}}$ introduced in Definition 2.7. As continuations map processes to rates, the relevant semi-ring is $\mathbb{R}_{\geq 0}$. The transition relation $\neg$ is the one induced by the rules of Fig. 6 plus rules (NIL), (CHO) and (CNS) of Fig. 3, with the obvious assumption that now $P, Q \in \mathcal{P}_{T I P P_{k}}$ and $\alpha \in \mathcal{L}_{T_{I P P_{k}}}$. Function $n$, in Fig. 6, maps each transition label to the relevant action, i.e. $n: \mathcal{L}_{T I P P_{k}} \rightarrow \mathcal{A}$, with $n \delta_{a}^{e}==_{\text {def }} a$. Functions $\mathcal{X}_{\mathbb{R}_{\geq 0}}$ and $\otimes_{\|_{L}}^{\mathbb{R}_{\geq 0}}$ are those introduced in Sect. 5.

Rule (PAR1) for interleaving ensures that all behaviors of $P \quad \|_{L} \quad Q$ after $\alpha$, whenever $(n \alpha) \notin L$, are either of the form $R \|_{L} Q$ where $P \stackrel{\alpha}{\mapsto} \mathscr{P}$ and $(\mathscr{P} R)>0$, for some $\mathscr{P}$, or of the form $P \|_{L} R$ where $Q \stackrel{\alpha}{\hookrightarrow} \mathscr{Q}$ and $(\mathscr{Q} R)>0$, for some $\mathscr{Q}$, as (a simple extension of what) we have seen in Sect. 5. Rule (PAR2 $\left.2_{\mathrm{T}}\right)$ for synchronization, instead, implements the rate multiplication principle of TIPP: if $(n \alpha) \in L, P \stackrel{\alpha}{\rightarrow} \mathscr{P}, Q \stackrel{\alpha}{\mapsto} \mathscr{Q},\left(\mathscr{P} R_{P}\right)=\lambda_{P}>0$, and $\left(\mathscr{Q} R_{Q}\right)=\lambda_{Q}>0$, then $P \|_{L} Q$ evolves, via $\alpha$, to $R_{P} \|_{L} R_{Q}$ with rate $\lambda_{P} \cdot \lambda_{Q}$.

The following proposition can be easily proven by structural induction:

Proposition 6.1. For all $P \in \mathcal{P}_{T_{I P P_{k}}}, \alpha \in \mathcal{L}_{T_{I P P_{k}}}$ and $\mathscr{P} \in \mathbf{T F}\left(\mathcal{P}_{T I P P_{k}}, \mathbb{R}_{\geq 0}\right)$, if $P \stackrel{\alpha}{\mapsto} \mathscr{P}$ can be derived using rules (NIL), (CHO) and (CNS) of Fig. 3, plus those of Fig. 6, then

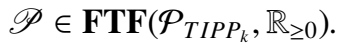

Definition 6.2. The formal semantics of $T I P P_{k}$ is the FsFUTS $\mathcal{R}_{T I P P_{k}}$ defined as the tuple $\left(\mathcal{P}_{T_{I P P_{k}}}, \mathcal{L}_{\text {TIPP }_{k}}, \mathbb{R}_{\geq 0}, \longmapsto\right)$, where $\lrcorner \subseteq \mathcal{P}_{T I P P_{k}} \times \mathcal{L}_{T_{I P P_{k}}} \times \mathbf{F T F}\left(\mathcal{P}_{T I P P_{k}}, \mathbb{R}_{\geq 0}\right)$ is the least relation induced by rules (NIL), (CHO) and (CNS) of Fig. 3 and by the rules in Fig. 6.

The following result characterizes the structure of $\mathcal{R}_{T I P P_{k}}$.

THEOREM 6.3. $\mathcal{R}_{\text {TIPP }_{k}}$ is total and deterministic.

The theorem below establishes, for $\operatorname{TIPP}_{k}$, the formal correspondence between the FUTS semantics and the original SOS, as in [Hermanns et al. 1998]. We recall here that $\mathbf{r t}_{a}(P, Q)$ is computed over the LTS characterized by the $\operatorname{TIPP}_{k}$ SOS and it yields the cumulative rate over the $a$-labelled transitions leading to $Q$ from $P$ in the LTS.

TheOREM 6.4. For all $P, Q \in \mathcal{P}_{T_{I P P_{k}}}, \alpha \in \mathcal{L}_{T_{I P P_{k}}}$, and unique $\mathscr{P} \in \mathbf{F T F}\left(\mathcal{P}_{\text {TIPP }_{k}}, \mathbb{R}_{\geq 0}\right)$ such that $P \stackrel{\alpha}{\rightarrow} \mathscr{P}:(\mathscr{P} Q)=\mathbf{r t}_{(n \alpha)}(P, Q)$.

A CTMC can be easily derived for each $P \in \mathcal{P}_{T I P P_{k}}$. We have to consider the FuTS generated from $P$, i.e. $\mathcal{R}_{T I P P_{k}} / P$, and for each $Q_{1}, Q_{2} \in \mathcal{P}_{T I P P_{k}} / P$ the rate matrix is defined as follows:

$$
\begin{aligned}
& \mathbf{R} Q_{1} Q_{2}=\sum_{\operatorname{def}} \sum_{\mathscr{P} \in\left\{\mathscr{P}^{\prime} \mid \exists \alpha \in \mathcal{L}_{T I P P_{k}} \cdot Q_{1} \stackrel{\alpha}{\mapsto} \mathscr{P}^{\prime}\right\}}\left(\mathscr{P} Q_{2}\right) \\
& \text { ACM Computing Surveys - In Press, Vol. , No., November } 2012 .
\end{aligned}
$$




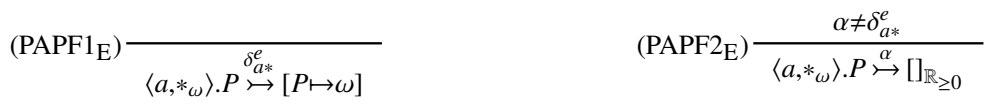

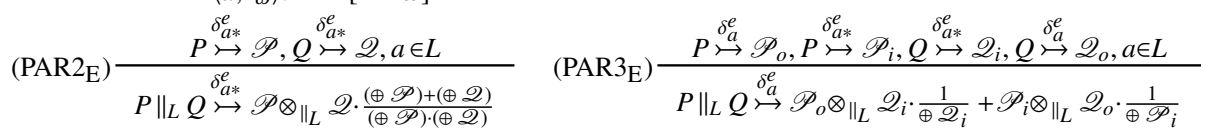

Fig. 7. Additional semantics rules for $\mathrm{EMPA}_{k}$

where, for notational simplicity, we have let $\rightarrow_{P}$ denote the transition relation of $\mathcal{R}_{T I P P_{k}} / P$. Notice that, by (syntactical) construction, for all $P \in \mathcal{P}_{T I P P_{k}}$ the set $\{\alpha \mid \exists \mathscr{P} . P \stackrel{\alpha}{\longmapsto} \mathscr{P}\}$ is finite; thus the above sum always converges.

\section{2 $\mathrm{EMPA}_{k}$}

In this section we consider EMPA [Aldini et al. 2010], but restrict our attention to the features of the exponentially timed kernel of EMPA and do not address other features of the language such as priorities, probabilities and immediate actions. The operators we consider are: inaction, rated-action prefix, passive-action prefix, choice, multi-party synchronization, and constant. The set $\mathcal{P}_{E M P A_{k}}$ of $\mathrm{EMPA}_{k}$ terms is induced by the grammar obtained by selecting from Fig. 2 the specific productions for the above operators, where $\omega \in \mathcal{W}_{E M P A}==_{\text {def }} \mathbb{R}_{>0}$.

Similarly to TIPP, EMPA associates delays to actions. The label set $\mathcal{L}_{E M P A_{k}}$ includes set $\Delta_{\mathcal{A}}$. Moreover, to model EMPA interactions that forbid synchronization between active actions, we let $\mathcal{L}_{E M P A_{k}}$ include labels explicitly indicating execution of passive actions. We let $\delta_{a *}^{e}$ denote the execution of passive action $a$ and let $\Delta_{\mathcal{A} *}$ be the set $\left\{\delta_{a *}^{e} \mid a \in \mathcal{A}\right\}$. Thus we have $\mathcal{L}_{E M P A_{k}}={ }_{\text {def }} \Delta_{\mathcal{A}} \cup \Delta_{\mathcal{A} *}$ and, in a similar way as in TIPP, we use function $n: \mathcal{L}_{E M P A_{k}} \rightarrow \mathcal{A}$ to obtain the action involved in the actual transition. The relevant semiring is $\mathbb{R}_{\geq 0}$. The transition relation $\neg$ is the one induced by rules of Fig. 7, plus Rules (NIL), (CHO) and (CNS) of Fig. 3 and Rules (RAPF1), (RAPF2) and (PAR1) of Fig. 6, where $P, Q \in \mathcal{P}_{E M P A_{k}}$ and $\alpha \in \mathcal{L}_{E M P A_{k}}$. In this SPC, each synchronization is obtained as the interaction of a single active action with a set of passive ones. The rate of the synchronization is that of the active action; passive actions are equipped with weights.

Rules $\left(\mathrm{PAPF} 1_{\mathrm{E}}\right)$ and $\left(\mathrm{PAPF} 2_{\mathrm{E}}\right)$ of Fig. 7 are self explanatory. Notice that, in Rule (PAR1) of Fig. 6, $\alpha$ can also be a delay of a passive action, $\delta_{a *}^{e}$ for some $a$, in which case $\mathscr{P}$ or $\mathscr{Q}$ yield weights. Rule (PAR2 $2_{\mathrm{E}}$ ) models the 'passive side' of EMPA's asymmetry principle for synchronization: for $a \in L$, if $P \stackrel{\delta_{a *}^{e}}{\rightarrow} \mathscr{P}, Q \stackrel{\delta_{a *}^{e}}{\rightarrow} \mathscr{Q},\left(\mathscr{P} R_{P}\right)=\omega_{P}>0$, and $\left(\mathscr{Q} R_{Q}\right)=\omega_{Q}>0$, then $P \|_{L} Q$ evolves to $R_{P} \quad \|_{L} R_{Q}$ with weight $\omega_{P} \cdot \omega_{Q} \cdot \frac{(\oplus \mathscr{P})+(\oplus \mathscr{Q})}{(\oplus \mathscr{P}) \cdot(\oplus \mathscr{Q})}$, under the assumption that the total weight of $a$ in $P($ i.e. $\oplus \mathscr{P})$ and the total weight of $a$ in $Q$ $($ i.e. $\oplus \mathscr{Q})$ are positive; otherwise $P \quad \|_{L} Q$ leads to [ $]_{\mathbb{R}_{\geq 0}}$ via $\delta_{a *}^{e}$. The normalization factor $\frac{(\oplus \mathscr{P})+(\oplus \mathscr{Q})}{(\oplus \mathscr{P P}) \cdot(\oplus \mathscr{Q})}$ is chosen, in EMPA, in such a way that the total weight of $a$ in $P \|_{L} Q$ is indeed $(\oplus \mathscr{P})+(\oplus \mathscr{Q})$. The second rule for synchronization, $\left(\mathrm{PAR}_{\mathrm{E}}\right)$, implements the asymmetry principle of EMPA: the transitions modeling the active role of $a$ in $P$, i.e. $P \stackrel{\delta_{a}^{e}}{\rightarrow} \mathscr{P}_{o}$, are paired with the transitions modeling the 'passive role' of $a$ in $Q$, i.e. $Q \stackrel{\delta_{a *}^{e}}{\rightarrow} \mathscr{Q}_{i}$, and the resulting function $\mathscr{P}_{o} \|_{L} \quad \mathscr{Q}_{i}$ is normalized with the positive weight of $a$ in $Q$, (i.e. $\oplus \mathscr{Q}_{i}$ ), and vice-versa. 
The following proposition can be easily proven by structural induction:

Proposition 6.5. For all $P \in \mathcal{P}_{E M P A_{k}}, \alpha \in \mathcal{L}_{E M P A_{k}}$, and $\mathscr{P} \in \mathbf{T F}\left(\mathcal{P}_{E M P A_{k}}, \mathbb{R}_{\geq 0}\right)$, if $P \stackrel{\alpha}{\mapsto} \mathscr{P}$ can be derived using the set of rules consisting of rules $(\mathrm{NIL}),(\mathrm{CHO})$ and $(\mathrm{CNS})$ of Fig. 3 plus rules (RAPF1), (RAPF2) and (PAR1) of Fig. 6 and the rules in Fig. 7, then $\mathscr{P} \in \mathbf{F T F}\left(\mathcal{P}_{E M P A_{k}}, \mathbb{R}_{\geq 0}\right)$.

Definition 6.6. The formal semantics of EMPA $A_{k}$ is the FsFuTS $\mathcal{R}_{E M P A_{k}}$ defined as the tuple $\left(\mathcal{P}_{E M P A_{k}}, \mathcal{L}_{E M P A_{k}}, \mathbb{R}_{\geq 0}, \mapsto\right)$ where the transition relation $\mapsto \subseteq \mathcal{P}_{E M P A_{k}} \times \mathcal{L}_{E M P A_{k}} \times$ $\operatorname{FTF}\left(\mathcal{P}_{E M P A_{k}}, \mathbb{R}_{\geq 0}\right)$ is the least relation satisfying the set of rules consisting only of the rules (NIL), (CHO) and (CNS) of Fig. 3 plus rules (RAPF1), (RAPF2) and (PAR1) of Fig. 6 and the rules of Fig. 7.

THEOREM 6.7. $\mathcal{R}_{E M P A_{k}}$ is total and deterministic.

The theorem below establishes, for $\mathrm{EMPA}_{k}$, the formal correspondence between the FuTS semantics and the original SOS, as in [Aldini et al. 2010]. We recall here that $\mathbf{r t}_{a}(P, Q)$ and $\mathbf{w t}_{a}(P, Q)$, are computed over the LTS characterized by the EMPA $k$ SOS and yield the cumulative rate and weight over the $a$-labelled transitions leading to $Q$ from $P$ in the LTS and weight $(P, a)$ is defined over the LTS as follows:

$$
\text { weight }(P, a)==_{\operatorname{def}} \sum\left\{\omega \in \mathbb{R}_{>0} \mid \exists P^{\prime} \in \mathcal{P}_{E M P A_{k}} . P \stackrel{a, *_{\omega}}{\longrightarrow} P^{\prime}\right\}
$$

TheOREM 6.8. For all $P, Q \in \mathcal{P}_{E M P A_{k}}, \delta_{a}^{e}, \delta_{a *}^{e} \in \mathcal{L}_{E M P A_{k}}$, and unique functions $\mathscr{P}, \mathscr{P}^{\prime} \in$ $\mathbf{F T F}\left(\mathcal{P}_{E M P A_{k}}, \mathbb{R}_{\geq 0}\right)$ such that $P \stackrel{\delta_{a}^{e}}{\rightarrow} \mathscr{P}$ and $P \stackrel{\delta_{a *}^{e}}{\longmapsto} \mathscr{P}^{\prime}$, the following holds: $(\mathscr{P} Q)=$ $\mathbf{r t}_{a}(P, Q),\left(\mathscr{P}^{\prime} Q\right)=\mathbf{w t}_{a}(P, Q)$, and $\left(\oplus \mathscr{P}^{\prime}\right)=\operatorname{weight}(P, a)$.

The CTMC associated with $P \in \mathcal{P}_{E M P A_{k}}$ is built by considering only the transitions associated with active actions. Consequently, the set of states is $\left(\mathcal{P}_{E M P A_{k}}\right) / P$, while the rate matrix is defined as follows:

$$
\mathbf{R} Q_{1} Q_{2}=\sum_{\operatorname{def}} \sum_{\mathscr{P} \in\left\{\mathscr{P}^{\prime} \mid \exists \alpha \in \Delta_{\mathcal{P}} \cdot Q_{1} \stackrel{\alpha}{\oplus} \mathscr{P}^{\prime}\right\}}\left(\mathscr{P} Q_{2}\right)
$$

We close this section by noting that the original syntax of EMPA contains also immediate actions, i.e. actions with no durations. We will show how to deal with this kind of actions when we consider the language IML (see Sect. 8) that clearly separates non-determinism and time and hence all its actions are durationless.

\section{3 $\mathrm{PEPA}_{k}$}

We consider now the kernel calculus $\mathrm{PEPA}_{k}$ of PEPA [Hillston 1996], consisting of ratedaction prefix, choice composition, multi-party synchronization, and constant.

The principle regulating the synchronization rate of PEPA processes is the so-called $\mathrm{min}$ imal rate, where, essentially, the rate of an action which is the result of the synchronization of two component processes is the MIN of the rates of synchronizing actions. Whenever a component process may perform the same action in several different ways, the cumulative, so-called apparent, rate has to be considered. The kernel we consider in this section is adequate for illustrating the minimal apparent rate principle; therefore, we leave out other features of PEPA like hiding. The set $\mathcal{P}_{P E P A_{k}}$ of $\mathrm{PEPA}_{k}$ terms is defined by the grammar obtained by selecting from Fig. 2 the specific productions for the above mentioned operators. 


$$
(\mathrm{PAR} 2 \mathrm{P}) \frac{P \stackrel{\alpha}{\longmapsto} \mathscr{P}, Q \stackrel{\alpha}{\mapsto} \mathscr{Q},(n \alpha) \in L}{P \|_{L} Q \stackrel{\alpha}{\longmapsto} \mathscr{P} \otimes_{\|_{L}} \mathscr{Q} \cdot \frac{\operatorname{MIN}\{\mathscr{P}, \oplus \mathscr{Q}\}}{\oplus \mathscr{P} \cdot \oplus \mathscr{Q}}}
$$

Fig. 8. Synchronization rule for $\mathrm{PEPA}_{k}$

Also in PEPA delays are associated with actions. Consequently, we let the label set $\mathcal{L}_{P E P A_{k}}$ be again the set $\Delta_{\mathcal{A}}$, ranged over by $\alpha, \alpha_{1}, \alpha^{\prime}, \ldots$; function $n$ for PEPA is defined as expected: $n: \mathcal{L}_{P E P A_{k}} \rightarrow \mathcal{A}$ with $n \delta_{a}^{e}={ }_{\text {def }} a$. The relevant set of states and semi-ring are $\mathcal{P}_{P_{E P A_{k}}}$ and $\mathbb{R}_{\geq 0}$, while the set of rules defining the transition relation $r$ is composed of the rule given in Fig. 8, plus Rules (CHO) and (CNS) of Fig. 3, and (RAPF1), (RAPF2) and (PAR1) of Fig. 6 where $P, Q \in \mathcal{P}_{P E P A_{k}}$ and $\alpha \in \mathcal{L}_{P E P A_{k}}$ must be assumed.

Rule (PAR2 ) for cooperation implements the minimal apparent rate principle: if $(n \alpha) \in$ $L, P \stackrel{\alpha}{\mapsto} \mathscr{P}, Q \stackrel{\alpha}{\mapsto} \mathscr{Q},\left(\mathscr{P} R_{P}\right)=\lambda_{P}>0$, and $\left(\mathscr{Q} R_{Q}\right)=\lambda_{Q}>0$, then $P \|_{L} Q$ evolves to $R_{P} \quad \|_{L} \quad R_{Q}$ with rate $\frac{\lambda_{P}}{\oplus \mathscr{P}} \cdot \frac{\lambda_{Q}}{\oplus \mathscr{Q}} \cdot \operatorname{MIN}\{\oplus \mathscr{P}, \oplus \mathscr{Q}\}$.

The following proposition can be easily proven by derivation induction:

Proposition 6.9. For all $P \in \mathcal{P}_{P_{E P A_{k}}}, \alpha \in \mathcal{L}_{P_{E P A_{k}}}$, and $\mathscr{P} \in \mathbf{T F}\left(\mathcal{P}_{P_{\text {EPA }}}, \mathbb{R}_{\geq 0}\right)$, if $P \stackrel{\alpha}{\longmapsto}$ $\mathscr{P}$ can be derived using the set of rules composed only of rules $(\mathrm{CHO})$ and $(\mathrm{CNS})$ of Fig. 3, rules (RAPF1), (RAPF2) and (PAR1) of Fig. 6 and rule $\left(P A R 2_{P}\right)$ of Fig. 8, then $\mathscr{P} \in \mathbf{F T F}\left(\mathcal{P}_{P_{E P A_{k}}}, \mathbb{R}_{\geq 0}\right)$.

Definition 6.10. The formal semantics of $P E P A_{k}$ is the FsFuTS $\mathcal{R}_{P E P A_{k}}$ defined as the tuple $\left(\mathcal{P}_{P E P A_{k}}, \mathcal{L}_{P E P A_{k}}, \mathbb{R}_{\geq 0}, \succ\right)$ where $\iota \subseteq \mathcal{P}_{P_{E P A_{k}}} \times \mathcal{L}_{P_{E P A_{k}}} \times \mathbf{F T F}\left(\mathcal{P}_{P E P A_{k}}, \mathbb{R}_{\geq 0}\right)$ is the least relation satisfying the set of rules consisting only of rules (CHO) and (CNS) of Fig. 3, (RAPF1), (RAPF2) and (PAR1) of Fig. 6 and rule (PAR2P) of Fig. 8.

THEOREM 6.11. $\mathcal{R}_{\text {EEPA }_{k}}$ is total and deterministic.

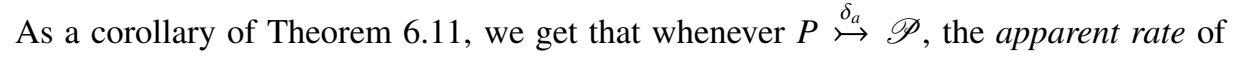
action $a$ in $P$, i.e. $r_{a}(P)=_{\operatorname{def}} \sum_{Q} \mathbf{r t}(P, Q)$, is given by $\oplus \mathscr{P}$.

The theorem below establishes, for $\mathrm{PEPA}_{k}$, the formal correspondence between the FuTS semantics and the original SOS, as in [Hillston 1996]. We recall here that $\mathbf{r t}_{a}(P, Q)$ is computed over the LTS characterized by the $\mathrm{PEPA}_{k}$ SOS and it yields the cumulative rate over the $a$-labelled transitions leading to $Q$ from $P$ in the LTS.

TheOREM 6.12. For all $P, Q \in \mathcal{P}_{P_{\text {EPA }} A_{k}}, \alpha \in \mathcal{L}_{P E P A_{k}}$, and unique $\mathscr{P} \in \mathbf{F T F}\left(\mathcal{P}_{P E P A_{k}}, \mathbb{R}_{\geq 0}\right)$ such that $P \stackrel{\alpha}{\rightarrow} \mathscr{P}$ the following holds: $(\mathscr{P} Q)=\mathbf{r t}_{(n \alpha)}(P, Q)$.

A CTMC can be associated with each $P \in \mathcal{P}_{P E P A_{k}}$ in a similar way as for $\operatorname{TIPP}_{k}$.

We close this section by observing that PEPA passive actions can be easily dealt with also in the FuTS approach. One way for doing this is to proceed as in [Hillston 1996], considering functions in $\mathbf{T F}\left(\mathcal{P}_{P_{E P A_{k}},}, \mathbb{R}_{\geq 0} \cup\left\{*_{\omega} \mid \omega \in \mathbb{N}_{>0}\right\}\right)$, using the equations for weights ${ }^{5}$. Moreover, weights are ordered as follows: $x<*_{\omega}\left(\forall x \in \mathbb{R}_{>0}\right), *_{\omega_{1}}<*_{\omega_{2}}$ if $\omega_{1}<\omega_{2}$. For details we refer the reader to [De Nicola et al. 2009c].

$5_{\omega_{\omega_{1}}}+* \omega_{\omega_{2}}=*_{\omega_{1}+\omega_{2}}, \frac{{ }^{*} \omega_{1}}{{ }^{*} \omega_{2}}=\frac{\omega_{1}}{\omega_{2}}$.

ACM Computing Surveys - In Press, Vol. , No. , November 2012. 


\section{FULLY MARKOVIAN CALCULI WITH ONE-TO-ONE SYNCHRONIZATION}

The SPCs that we have considered in the previous sections all rely on an operator for multi-party parallel composition. In this section, we consider stochastic extensions of CCS [Milner 1989] that, instead, is based on a one-to-one, sometimes called binary, interaction paradigm. A synchronization between processes $P$ and $Q$, running in parallel, occurs when $P$ sends a signal over a channel (action $\bar{a}$ ) and $Q$ receives a signal over the same channel (action $a$ ). While there have been many variants of stochastic calculi based on the multi-party interaction paradigm, very few proposals have been put forward for the CCS based one. Moreover, all of them are inspired by [Priami 1995], that introduces a stochastic extension of $\pi$-calculus, a calculus for mobility that generalizes CCS and guarantees a sophisticated handling of channel names and their visibility. In this section, we consider two stochastic extensions of CCS that in general terms take inspiration from the approach presented in [Priami 1995].

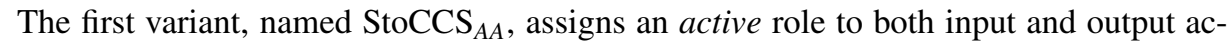
tions. Following a similar approach as that used by Klin and Sassone [2008], we consider two alternatives ways of computing the rate of a one-to-one synchronization. In the first one, like in TIPP, the rate of a synchronization is obtained as the product of the rates of the involved input and output actions. In the second one, the rate of a synchronization is computed $a$ là PEPA, like in [Priami 1995] for the $\pi$-calculus, and is obtained as the minimum between the total input and the total output rates over the same channel. We shall see that in the second case associativity of parallel composition is lost.

The second variant, named StoCCS $A P$, follows an approach similar to the calculus EMPA that we considered in Sect.6.2: it is assumed that output actions have an active role while input actions are passive. The rate of a synchronization is then the one of the involved output action. This simple stochastic extension permits highlighting some of the intricacies related to stochastic extensions of CCS-like calculi. We will see that differently from the multiparty synchronization approach, where synchronizations have a local nature, in the one-to-one synchronization approach, synchronizations play a global role. This means that, in order to guarantee desirable properties, e.g. associativity of parallel composition, re-normalizations of synchronization rates are necessary.

We will conclude this section with a discussion on associativity of one-to-one synchronization operators when both input and output actions are considered active and the minimal rate principle is used. It will be shown that an associative one-to-one synchronization parallel composition operator implementing the minimal rate principle can be defined. This somehow contradicts the result of Klin and Sassone [2008] according to which, within the SGSOS framework, associativity of CCS parallel composition can be guaranteed only when the multiplicative approach is used ${ }^{6}$. We discuss the additional information that needs to be kept into account in order to overcome the obstacles.

\subsection{Active-active synchronization}

In this section we consider StoCCS $\mathrm{CA}_{A}$ and the two variants for computing synchronization rates. The syntax of the language consists of the following operators: inaction, ratedoutput-action prefix, rated-input-action prefix, choice, and binary synchronization. Con-

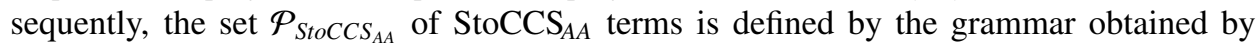

${ }^{6}$ SGSOS is a syntactic framework for the definition of the SOS of SPCs proposed by Klin and Sassone [2008] in analogy to standard GSOS [Aceto et al. 2001]. 


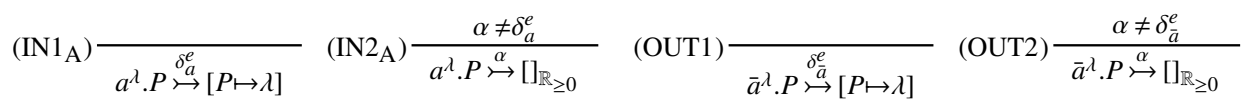

$$
\begin{aligned}
& (\mathrm{PAR} 1) \frac{P \stackrel{\alpha}{\mapsto} \mathscr{P}, Q \stackrel{\alpha}{\mapsto} \mathscr{Q}, \alpha \neq \delta_{\overparen{a}}^{e}}{P \mid Q \stackrel{\alpha}{\mapsto}\left(\mathscr{P} \otimes_{\mid}(X Q)\right)+\left((X P) \otimes_{\mid} \mathscr{Q}\right)}
\end{aligned}
$$

Fig. 9. Additional semantic rules for $\operatorname{StoCCS}_{A A}$

selecting from Fig. 2 the productions specific to the above mentioned operators.

We let the label set $\mathcal{L}_{\text {StoCCS }}$ be defined as the set $\Delta_{\mathcal{A}} \cup \Delta_{\overline{\mathcal{A}}} \cup \Delta_{\overline{\mathcal{A}}}$, where $\Delta_{\overline{\mathcal{A}}}$ and $\Delta_{\overline{\mathcal{A}}}$ are the sets $\left\{\delta_{a}^{e} \mid a \in \overline{\mathcal{A}}\right\}$ and $\left\{\delta_{\widehat{a}}^{e} \mid a \in \mathcal{A}\right\}$, respectively. $\Delta_{\widehat{\mathcal{A}}}$ is the set of synchronizations on channels; $\delta_{\widehat{a}}^{e}$ denotes a synchronization on channel $a$, which in CCS is more abstractly denoted by $\tau$. The additional information, i.e. the name of the synchronization channel, is crucial for the associativity result. The relevant semi-ring is $\mathbb{R}_{\geq 0}$.

The transition relation $\longmapsto$ for the multiplicative synchronization variant is the one induced by rules in Fig. 9 and Fig. 10, plus Rules (NIL) and (CHO) of Fig. 3, where $P, Q \in \mathcal{P}_{\text {StoCCS }}$ AA and $\alpha \in \mathcal{L}_{\text {StoCCS }}$ AA is assumed. The rules for the minimal rate variant are as above but with the rule of Fig. 11 replacing that in Fig. 10.

Rules $\left(\mathrm{IN} 1_{\mathrm{A}}\right)$ and $\left(\mathrm{IN} 2_{\mathrm{A}}\right)$ ((OUT1) and (OUT2) respectively) govern the behavior of input actions (output actions respectively), while rule (PAR1) describes interleaving behavior of parallel processes. Notice that, rule (PAR1) can only be applied when the involved label $\alpha$ is not a synchronization, i.e. when $\alpha \neq \delta_{\vec{a}}^{e}$.

Rules for synchronization deserve a few remarks. In the following we first discuss the multiplicative variant, obtained when using Rule (PAR $\mathrm{PA}_{\mathrm{A}-\mathrm{mul}}$ ) of Fig. 10, and then the

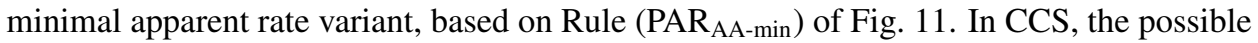
continuations of $P \mid Q$ after a synchronization $\widehat{a}$ are the following:

(1) the continuations of $P$ after $\widehat{a}$, in parallel with $Q$;

(2) the continuations of $Q$ after $\widehat{a}$, in parallel with $P$;

(3) the continuations of $P$ after $a$ in parallel with continuations of $Q$ after $\bar{a}$;

(4) the continuations of $P$ after $\bar{a}$ in parallel with the continuations of $Q$ after $a$.

Notice that the synchronizations $\widehat{a}$ considered in case (1) occur internally in $P$ alone, i.e. without the cooperation of $Q$; symmetrically, the synchronizations $\widehat{a}$ considered in case (2) occur internally in $Q$ alone, i.e. without the cooperation of $P$. Let continuation functions $\mathscr{P}, \mathscr{P}_{i}$ and $\mathscr{P}_{o}$ (respectively $\mathscr{Q}, \mathscr{Q}_{i}$ and $\mathscr{Q}_{o}$ ) be associated with $P$ (respectively $Q$ ) after an internal synchronization, an input and an output over channel $a$. When the multiplicative approach is used to compute the synchronization rates, continuations (1)-(4) described above can easily be computed by means of the parallel aggregation and characteristic functions $\left(\otimes_{\mid}\right.$and $\mathcal{X}$, respectively) introduced in Sect. 5, as follows:

(1) $\left(\mathscr{P} \otimes_{\mid}(X Q)\right)$ is the continuation of $P$ after $\delta_{\widehat{a}}^{e}$ (a synchronization on $a$, which occurs internally in $\mathrm{P})$, in parallel with $Q$;

(2) $\left((X P) \otimes_{\mid} \mathscr{Q}\right)$ is the continuation of $Q$ after $\delta_{\widehat{a}}^{e}$ (a synchronization on $a$, which occurs internally in $\mathrm{Q})$, in parallel with $P$;

(3) $\mathscr{P}_{i} \otimes_{\mid} \mathscr{Q}_{o}$ is the continuation after composing an input in $P$ with an output in $Q$, over channel $a$;

ACM Computing Surveys - In Press, Vol. , No., November 2012. 


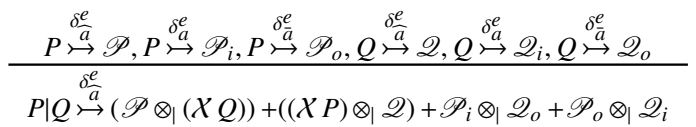

Fig. 10. Rule (PAR AA-mul) for multiplicative synchronization for StoCCS $_{A A}$

(4) $\mathscr{P}_{o} \otimes_{\mid} \mathscr{Q}_{i}$ is the continuation after composing an output in $P$ with an input in $Q$, over channel $a$.

Notice that in the latter two cases, synchronization rates are obtained as the multiplication between the involved output and input rates. These are all the basic ingredients of Rule (PAR AA-mul $\left._{1}\right)$, shown in Fig. 10.

For instance, if we consider process $P$ defined as $\bar{a}^{\lambda_{1}}$.nil $\mid a^{\lambda_{2}}$.nil, continuation functions as (1)-(4) above are:

(1) []$\otimes_{\mid}\left(X a^{\lambda_{2}}\right.$.nil $)=[] \otimes_{\mid}\left[a^{\lambda_{2}}\right.$.nil $\left.\rightarrow 1\right]=[]$;

(2) $\left(X \bar{a}^{\lambda_{2}}\right.$.nil $) \otimes_{\mid}[]=\left[\bar{a}^{\lambda_{2}}\right.$.nil $\left.\rightarrow 1\right] \otimes_{\mid}[]=[]$;

(3) []$\otimes_{1}[]=[]$;

(4) $\left[\right.$ nil $\left.\rightarrow \lambda_{1}\right] \otimes_{\mid}\left[\right.$nil $\left.\rightarrow \lambda_{2}\right]=\left[\right.$ nil $\mid$ nil $\left.\rightarrow \lambda_{1} \cdot \lambda_{2}\right]$

Summarizing, $P \stackrel{\delta^{e}}{\rightarrow}\left[\right.$ nil $\mid$ nil $\left.\rightarrow \lambda_{1} \cdot \lambda_{2}\right]$. Let us now consider $P \mid a^{\lambda_{3}}$.nil. The derivation for such a process will be based on the continuations below:

(1) $\left[\right.$ nil $\mid$ nil $\left.\rightarrow \lambda_{1} \cdot \lambda_{2}\right] \otimes_{\mid}\left(X a^{\lambda_{3}}\right.$.nil $)=\left[(\right.$ nil $\mid$ nil $) \mid a^{\lambda_{3}}$.nil $\left.\rightarrow \lambda_{1} \cdot \lambda_{2}\right]$;

(2) $(X P) \otimes_{\mid}[]=[]$;

(3) $\left[\bar{a}^{\lambda_{1}}\right.$.nil $\mid$ nil $\left.\rightarrow \lambda_{2}\right] \otimes_{\mid}[]=[]$;

(4) $\left[\right.$ nil $\mid a^{\lambda_{2}}$.nil $\left.\rightarrow \lambda_{1}\right] \otimes_{\mid}\left[\right.$nil $\left.\rightarrow \lambda_{3}\right]=\left[\left(\right.\right.$ nil $\mid a^{\lambda_{2}}$.nil $) \mid$ nil $\left.\rightarrow \lambda_{1} \cdot \lambda_{3}\right]$;

Summing up these continuations, we obtain:

$$
\left[(\text { nil } \mid \text { nil }) \mid a^{\lambda_{3}} \text {.nil } \rightarrow \lambda_{1} \cdot \lambda_{2},\left(\text { nil } \mid a^{\lambda_{2}} \text {.nil }\right) \mid \text { nil } \rightarrow \lambda_{1} \cdot \lambda_{3}\right]
$$

that is: $P \mid a^{\lambda_{3}}$.nil $\stackrel{\delta_{a}^{e}}{\rightarrow}\left[(\right.$ nil $\mid$ nil $) \mid a^{\lambda_{3}}$.nil $\rightarrow \lambda_{1} \cdot \lambda_{2},\left(\right.$ nil $\mid a^{\lambda_{2}}$.nil $) \mid$ nil $\left.\rightarrow \lambda_{1} \cdot \lambda_{3}\right]$.

A more complicated rule has to be used to handle synchronizations where the minimal rate approach is used. Let $\mathscr{P}_{i}$ be the continuation of $P$ after an input over $a$, and $\mathscr{Q}_{o}$ be the continuation of $Q$ after a $\bar{a}$, then the rate of a synchronization on channel $a$ between an input in $P$ and an output in $Q$ is obtained as the minimum of their apparent rates, i.e. the minimum of the total rate of $a$-inputs in $P\left(\oplus \mathscr{P}_{i}\right)$ and the total rate of $\bar{a}$-outputs in $Q\left(\oplus \mathscr{Q}_{o}\right)$. The synchronizations between output in $P$ and input in $Q$ are dealt with similarly. As we know, a specific process $P^{\prime}$ is reached from $P$, after input over $a$, with rate $\left(\mathscr{P}_{i} P^{\prime}\right)$; similarly, $Q^{\prime}$ is reached from $Q$, after output $\bar{a}$, with rate $\left(\mathscr{Q}_{o} Q^{\prime}\right)$; thus the probability that such a specific interaction takes place is $\frac{\left(\mathscr{P}_{i} P^{\prime}\right) \cdot\left(\mathscr{Q}_{o} Q^{\prime}\right)}{\oplus \mathscr{P}_{i} \cdot \mathscr{Q}_{o}}$. Hence, the final synchronization rate is $\frac{\left(\mathscr{P}_{i} P^{\prime}\right) \cdot\left(\mathscr{Q}_{o} Q^{\prime}\right)}{\oplus \mathscr{P}_{i} \oplus \mathscr{Q}_{o}} \cdot \operatorname{MIN}\left\{\oplus \mathscr{P}_{i}, \oplus \mathscr{Q}_{o}\right\}$. Notice that $\left(\mathscr{P}_{i} P^{\prime}\right) \cdot\left(\mathscr{Q}_{o} Q^{\prime}\right)$ is the synchronization rate used in the multiplicative rate approach. So we can obtain the rule for the minimal rate approach from that of the multiplicative rate approach; we only have to modify rule $\left(\mathrm{PAR}_{\mathrm{AA}-\mathrm{mul}}\right)$ in order to re-normalize $\mathscr{P}_{i} \otimes_{\mid} \mathscr{Q}_{o}$ and $\mathscr{P}_{o} \otimes_{\mid} \mathscr{Q}_{i}$ using $\frac{\operatorname{Min}\left\{\oplus \mathscr{P}_{i}, \oplus \mathscr{Q}_{o}\right\}}{\oplus \mathscr{P}_{i} \oplus \mathscr{Q}_{o}}$ and $\frac{\operatorname{MiN}\left\{\oplus \mathscr{P}_{o} \oplus \mathscr{Q}_{i}\right\}}{\oplus \mathscr{P}_{o} \oplus \oplus \mathscr{Q}_{i}}$ respectively. All the above is formalized in Rule (PAR $\mathrm{PA}-m i n)$ shown in Fig. 11. 


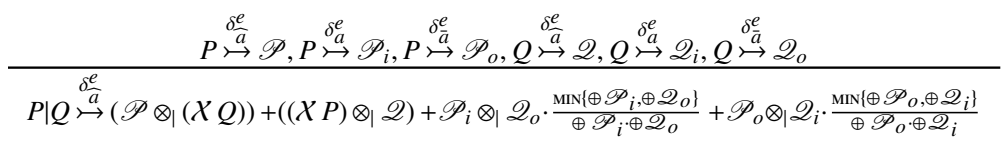

Fig. 11. Rule (PAR $\mathrm{AA-min})$ for minimal rate synchronization for $\mathrm{StoCCS}_{A A}$

Proposition 7.1. For all $P \in \mathcal{P}_{\text {StoCCS }_{A A}}, \alpha \in \mathcal{L}_{\text {StoCCS }}$, and $\mathscr{P} \in \mathbf{T F}\left(\mathcal{P}_{\text {StoCCS }_{A A}}, \mathbb{R}_{\geq 0}\right)$ if $P \stackrel{\alpha}{\longmapsto} \mathscr{P}$ can be derived using the set of rules consisting of rules (NIL) and (CHO) of Fig. 3, plus rules in Fig. 9, and using one out of $\left(P A R_{A A-m u l}\right)$ and $\left(P A R_{A A-m i n}\right)$, then $\mathscr{P} \in \mathbf{F T F}\left(\mathscr{P}_{S t o C C S_{A A}}, \mathbb{R}_{\geq 0}\right)$.

Below we define the two FuTSs corresponding to the two variants of the StoCCS $A A$ when the multiplicative or the minimal rate synchronization approach is taken.

Definition 7.2 Formal FuTS semantics of StoCCS $S_{A A}$.

(1) The semantics of the multiplicative synchronization variant of StoCCS SA $_{\text {is }}$ is the FsFTS $\mathcal{R}_{\text {StoCCS }}^{\text {muA }}=_{\text {def }}\left(\mathcal{P}_{\text {StoCCS }}, \mathcal{L}_{\text {StoCCS }}, \mathbb{R}_{\geq 0}, \longmapsto\right)$ where the transition relation $\neg \subseteq$ $\mathcal{P}_{\text {StoCCS }}{ }_{A A} \times \mathcal{L}_{\text {StoCCS }_{A A}} \times \mathbf{F T F}\left(\mathcal{P}_{\text {StoCCS }}, \mathbb{R}_{\geq 0}\right)$ is the least relation satisfying only rules (NIL) and (CHO) of Fig. 3, the rules in Fig. 9, and rule (PAR AA-mul $)$.

(2) The formal semantics of the minimal rate synchronization variant of StoCCS $_{A A}$ is the FsFuTS $\mathcal{R}_{\text {StoCCS }}^{\min }=_{\text {def }}\left(\mathcal{P}_{\text {StoCCS }}, \mathcal{L}_{\text {StoCCS }}, \mathbb{R}_{\geq 0}, \longmapsto\right)$ where the transition relation $\longmapsto \subseteq \mathcal{P}_{S_{t o C C S_{A A}}} \times \mathcal{L}_{S_{\text {to } C C S_{A A}}} \times \mathbf{F T F}\left(\mathcal{P}_{\text {StoCCS }_{A A}}, \mathbb{R}_{\geq 0}\right)$ is the least relation satisfying only rules (NIL) and (CHO) of Fig. 3, the rules in Fig. 9, and rule (PAR AA-min $)$.

TheOREM 7.3. $\mathcal{R}_{\text {StoCCS }}^{\text {mul }}$ and $\mathcal{R}_{\text {StoCCS }}^{\text {min }}$ are total and deterministic.

The following theorem establishes the formal correspondence between the FuTS semantics of StoCCS ${ }_{A A}$ and the semantics definition of Stochastic CCS a là Priami [1995], as reported in Section 2.2 of [Klin and Sassone 2008].

Theorem 7.4. For all $P, Q \in \mathcal{P}_{\text {StoCCS }_{A A}}, \alpha \in \mathcal{L}_{\text {StoCCS }}$, and unique function $\mathscr{P}$ in $\mathbf{F T F}\left(\mathcal{P}_{\text {StoCCS }}, \mathbb{R}_{\geq 0}\right)$ such that $P \stackrel{\alpha}{\longmapsto} \mathscr{P}$ the following holds:

$$
(\mathscr{P} Q)=\left\{\begin{array}{l}
\mathbf{r t}_{a}(P, Q), \text { if } \alpha=\delta_{a}^{e} \text { with } a \in \mathcal{A} \cup \overline{\mathcal{A}}, \\
\mathbf{r t}_{\langle a \mid \bar{a}\rangle}(P, Q)+\mathbf{r t}_{\langle\bar{a} \mid a\rangle}(P, Q), \text { if } \alpha=\delta_{\bar{a}}^{e} \text { with } a \in \mathcal{A} .
\end{array}\right.
$$

As pointed out by Klin and Sassone [2008], Stochastic CCS with a minimal apparent rate semantics suffers of non-associativity of the parallel composition operator, with respect to strong Markovian bisimilarity ${ }^{7}$. Intuitively, the problem is that terms which differ only for the grouping of parallel components generate transitions which correspond to 'the same' interactions but assign different rates to 'the same' continuation behaviors.

As an example (taken from [Klin and Sassone 2008]), let us consider processes $P$ and $Q$, where $P$ is the term $\left(P_{1} \mid P_{2}\right) \mid P_{3}$ and $Q$ is the term $P_{1} \mid\left(P_{2} \mid P_{3}\right)$ where $P_{1}, P_{2}$ and $P_{3}$ are the terms $a^{\lambda}$.nil, $a^{\lambda}$.nil and $\bar{a}^{\lambda}$.nil, respectively. We have that process $P$ gives rise to a single $\delta_{\widehat{a}}^{e}$-labelled transition leading to the following continuation function:

$$
\left[\left(P_{1} \mid \text { nil }\right) \mid \text { nil } \mapsto \frac{\lambda}{2},\left(\text { nil } \mid P_{2}\right) \mid \text { nil } \mapsto \frac{\lambda}{2}\right]
$$

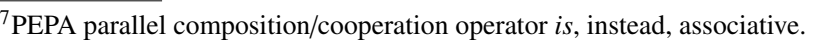

ACM Computing Surveys - In Press, Vol. , No. , November 2012. 


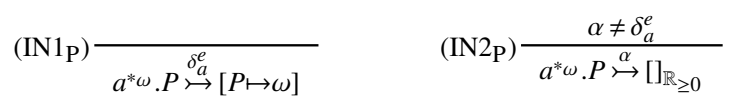

Fig. 12. Semantic rules for StoCCS $\mathrm{SP}_{A}$

while process $Q$ gives rise to a single $\delta_{\vec{a}}^{e}$-labelled transition leading to the following continuation function:

$$
\left[P_{1} \mid(\text { nil } \mid \text { nil }) \mapsto \lambda, \text { nil } \mid\left(P_{2} \mid \text { nil }\right) \mapsto \lambda\right]
$$

Clearly, process $P$, after an interaction between $P_{2}$ and $P_{3}$, reaches $\left(P_{1} \mid\right.$ nil $) \mid$ nil with rate $\frac{\lambda}{2}$, while process $Q$, after the same interaction, reaches $P_{1} \mid$ (nil $\mid$ nil), with rate $\lambda$. Thus, the stochastic behavior of $P$ and $Q$ is different. The basic reason for the difference is the fact that, in $Q$, the rate of the synchronization between $P_{2}$ and $P_{3}$ is computed without taking into account the presence of the input action in $P_{1}$, as is instead the case for $P$.

From the results in [Klin and Sassone 2008] it follows that it is impossible to define an SGSOS semantics that guarantees the associativity of CCS parallel composition. In the next section, however, we discuss how this problem can be overcome in the FuTS approach.

\subsection{Active-passive synchronization}

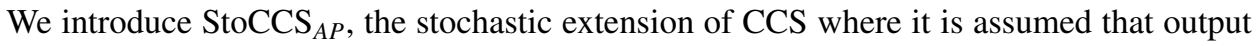
actions have an active role while input actions are considered as passive. The duration of a synchronization is determined by the rate assigned to the participating output action. Input actions are annotated with weights, i.e. positive integers that are only used for determining the probability that a specific input action is selected among the possible ones when a complementary output action is executed. This approach is inspired by the notion of passive actions of EMPA discussed in Sect. 6.2.

The set $\boldsymbol{P}_{S t o C C S_{A P}}$ of $\mathrm{StoCCS}_{A P}$ terms are obtained by considering: inaction, ratedoutput-action prefix, passive-input-action prefix, choice, and binary synchronization. Consequently, the set $\mathcal{P}_{S t o C C S_{A P}}$ of StoCCS ${ }_{A P}$ terms is defined by the grammar obtained by selecting from Fig. 2 the productions specific of the above mentioned operators where the additional constraint is imposed that the two processes in a nondeterministic term of the form $P+Q$ cannot offer alternative input and output actions on the same channel. In other words, processes of the form $\bar{a}^{\lambda} \cdot P_{1}+a^{* \omega} \cdot Q_{1}$ are not allowed. This is mainly for the sake of simplicity; otherwise the computation of the synchronization rates would be technically more complicated because we would have to take care that no synchronization erroneously occurred between the alternative components of a choice term.

The label set $\mathcal{L}_{S t o C C S_{A P}}$ is the same as the one for StoCCS $S_{A A}$, i.e. $\Delta_{\mathcal{A}} \cup \Delta_{\overline{\mathcal{A}}} \cup \Delta_{\overline{\mathcal{F}}}$. The transition relation $\neg$ is the one induced by the rules in Fig. 12, modeling the behavior of passive input actions, plus the rule governing the synchronization that will be introduced below, plus Rules (NIL) and (CHO) of Fig. 3, (OUT1), (OUT2) and (PAR1) in Fig. 9, where $P, Q \in \mathcal{P}_{S t o C C S_{A P}}$ and $\alpha \in \mathcal{L}_{S t o C C S_{A P}}$ is assumed.

The rule for synchronization deserves more attention and specific motivations. The next states of $P \mid Q$ after $\widehat{a}$, i.e. after a synchronization over channel $a$ has taken place, can be obtained by composing next state functions $\mathscr{P}, \mathscr{P}_{i}$ and $\mathscr{P}_{o}\left(\mathscr{Q}, \mathscr{Q}_{i}\right.$, and $\mathscr{Q}_{o}$, respectively) associated with $P$ (respectively $Q$ ) after a synchronization internal to $P(Q$, respectively), after an input and after an output over channel $a$, by using parallel aggregation $\left(\otimes_{\mid}\right)$, renor- 
malization $\left({ }_{-} \mathbb{R}_{-} /{ }_{-}\right)$and characteristic $(\mathcal{X})$ functions introduced in Sect. 5.

A straightforward implementation of the synchronization rule would take into account the following components when calculating the continuations of $P \mid Q$ after $\widehat{a}$ :

(1) the continuations of $P$ after $\widehat{a}$, in parallel with $Q$, i.e. $\mathscr{P} \otimes_{\mid}(X Q)$;

(2) the continuations of $Q$ after $\widehat{a}$, in parallel with $P$, i.e. $(X P) \otimes_{\mid} \mathscr{Q}$;

(3) the continuations of $P$ after $a$ in parallel with the continuations of $Q$ after $\bar{a}$, renormalized w.r.t. the total weight of inputs in $P$, i.e. $\frac{\mathscr{P}_{i} \otimes_{\otimes^{\mathrm{R}}} \mathscr{Q}_{o}}{\oplus \mathscr{P}_{i}}$;

(4) the continuations of $P$ after $\bar{a}$ in parallel with the continuations of $Q$ after $a$, renormalized w.r.t. the total weight of inputs in $Q$, i.e $\frac{\mathscr{P}_{o} \otimes_{1}^{\mathrm{R}} \mathscr{Q}_{i}}{\oplus \mathscr{Q}_{i}}$.

Renormalization in (3) and (4) is needed to correctly compute the relative probability of selecting a specific input action. Notice, in fact, that in the absence of such a renormalization, the exit rate of the state preceding the synchronization would increase with the number of (passive!) input possibilities.

Formally, the rule corresponding to the composition of the four components intuitively described above is the following:

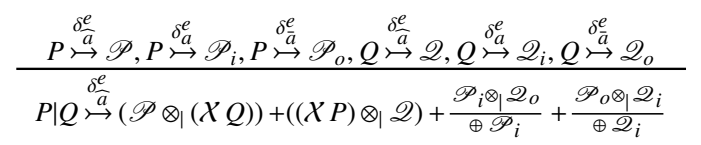

Unfortunately, if we use this rule, associativity of parallel composition is again lost. To see this, just reconsider, in the context of StoCCS ${ }_{A P}$, the example discussed in the previous section. Let $P$ and $Q$ be as before, but with $P_{1}, P_{2}$ and $P_{3}$ being the terms $a^{*_{\omega}}$.nil, $a^{*_{\omega}}$.nil, and $\bar{a}^{\lambda}$.nil respectively. As before, $P$ reaches both $\left(\right.$ nil $\left.\mid P_{2}\right) \mid$ nil and $\left(P_{1} \mid\right.$ nil $) \mid$ nil with rate $\frac{\lambda}{2}$ while $Q$ reaches $P_{1} \mid$ (nil $\mid$ nil) and nil $\mid\left(P_{2} \mid\right.$ nil) with rate $\lambda$. However, when using the active-passive approach, this problem can be easily overcome. Let us consider, for instance, a synchronization $\widehat{a}$ taking place internally within $P$ alone which will be 'inherited' by $P \mid Q$, i.e. will appear as a synchronization $\widehat{a}$ in the parallel composition (in practice, we are focussing on the first term of the sum in the continuation function in the sequent of the rule above). This synchronization originates from a specific output action $\bar{a}$ of $P$. When considering this output in the context of the parallel composition $P \mid Q$, we have to take into account that such an output could have synchronized with an input action $a$ in $Q$ instead of one in $P$. More precisely when we consider $P$ alone, the rate of such an interaction is computed by multiplying the rate of the output $\bar{a}$ by the weight of the specific input $a$ selected for the interaction and dividing by the total weight of input on $a$ within $P$ (i.e. $\oplus \mathscr{P}_{i}$ ). When, instead, we consider the same output action giving rise to interactions within the broader context $P \mid Q$, we have to (multiply the rate and the weight as before and) divide by the total weight of input on a within $P \mid Q$ (i.e. $\oplus \mathscr{P}_{i}+\oplus \mathscr{Q}_{i}$ ). In order to recover the original value, i.e. the rate of the output $\bar{a}$ multiplied by the weight of the specific input $a$, as above, and to renormalize it w.r.t. the global context $P \mid Q$, all we need to do is to use $\frac{\oplus \mathscr{P}_{i}}{\oplus \mathscr{P}_{i}+\oplus \mathscr{Q}_{i}}$ as normalization factor for the rates in $\mathscr{P}$. Thus, the component of the continuation function related to synchronizations $\widehat{a}$ in $P \mid Q$ taking place internally in $P$ is $\frac{\left(\mathscr{P} \otimes_{(}(X Q)\right) \cdot \oplus \mathscr{P}_{i}}{\oplus \mathscr{P}_{i}+\oplus \mathscr{Q}_{i}}$. Symmetrically, we get that the component of the continuation function related to synchronizations $\widehat{a}$ in $P \mid Q$ taking place internally in $Q$ is $\frac{\left.((X P)) \otimes_{1} \mathscr{Q}\right) \cdot \oplus \mathscr{Q}_{i}}{\oplus \mathscr{P}_{i}+\oplus \mathscr{Q}_{i}}$. For similar considerations, when considering $\widehat{a}$ synchronizations originating from outputs $\bar{a}$ in 


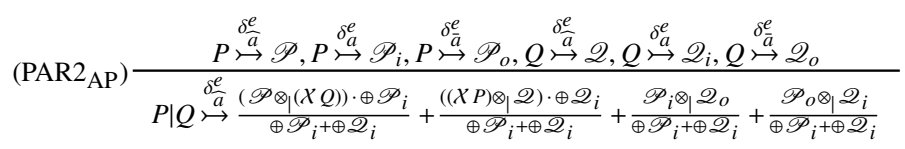

Fig. 13. Rule (PAR2 $\mathrm{AP})$ for rate synchronization for $\mathrm{StoCCS}_{A P}$

$Q$ (in $P$, respectively) and inputs $a$ in $P$ (in $Q$, respectively), the normalization factor must be $\oplus \mathscr{P}_{i}+\oplus \mathscr{Q}_{i}$ and not simply $\oplus \mathscr{P}_{i}\left(\oplus \mathscr{Q}_{i}\right.$ respectively). Notice that the requirement of no input-output mix in choice terms guarantees that only the rates of those input actions which can actually take part in a synchronization are considered in the normalization factor. The resulting new rule for synchronization is reported in Fig. 13. Applying the new semantics to the above example, we get the following two transitions:

(1) $\left(P_{1} \mid P_{2}\right) \mid P_{3} \stackrel{\delta_{\vec{a}}^{e}}{\longmapsto}\left[\left(\right.\right.$ nil $\left.\mid P_{2}\right) \mid$ nil $\mapsto \frac{\lambda}{2},\left(P_{1} \mid\right.$ nil $) \mid$ nil $\left.\mapsto \frac{\lambda}{2}\right]$

(2) $P_{1} \mid\left(P_{2} \mid P_{3}\right) \stackrel{\delta_{a}^{e}}{\longmapsto}\left[\right.$ nil $\mid\left(P_{2} \mid\right.$ nil $) \mapsto \frac{\lambda}{2}, P_{1} \mid($ nil $\mid$ nil $\left.) \mapsto \frac{\lambda}{2}\right]$

Proposition 7.5. For all $P \in \mathcal{P}_{\text {StoCCS }_{A P}}, \alpha \in \mathcal{L}_{\text {StoCCS }}$, and $\mathscr{P} \in \mathbf{T F}\left(\mathcal{P}_{\text {StoCCS }_{A P}}, \mathbb{R}_{\geq 0}\right)$, if $P \stackrel{\alpha}{\longmapsto} \mathscr{P}$ can be derived using the set of rules consisting of rules (NIL) and (CHO) of Fig. 3, rules (OUT1), (OUT2) and (PAR1) in Fig. 9, rules $\left(I N 1_{P}\right),\left(I N 2_{P}\right)$ in Fig. 12 and rule $\left(P A R 2_{A P}\right)$ of Fig. 13, then $\mathscr{P} \in \mathbf{F T F}\left(\mathscr{P}_{S t o C C S_{A P}}, \mathbb{R}_{\geq 0}\right)$.

Definition 7.6. The formal semantics of StoCCS $S_{A P}$ is the FsFuTS $\mathcal{R}_{\text {StoCCS }}$ defined as $\left(\mathcal{P}_{\text {StoCCS }}, \mathcal{L}_{\text {StoCCS }}, \mathbb{R}_{\geq 0}, \mapsto\right)$ where $\mapsto \subseteq \mathcal{P}_{\text {StoCCS }} \times \mathcal{L}_{\text {StoCCS }} \times \mathbf{F T F}\left(\mathcal{P}_{\text {StoCCS }}, \mathbb{R}_{\geq 0}\right)$ is the least relation satisfying the set of rules consisting of rules (NIL) and (CHO) of Fig. 3 , rules (OUT1), (OUT2) and (PAR1) in Fig. 9, rules $\left(\mathrm{IN} 1_{\mathrm{P}}\right),\left(\mathrm{IN} 2_{\mathrm{P}}\right)$ in Fig. 12 and rule $(\mathrm{PAR} 2 \mathrm{AP})$ of Fig. 13.

THEOREM 7.7. $\mathcal{R}_{\text {StoCCS }_{A P}}$ is total and deterministic.

De Nicola et al. [2009c] showed that, by using the above rule, associativity of parallel composition is guaranteed:

Theorem 7.8. For all $P, Q, R \in \mathcal{P}_{\text {StoCCS }_{A P}},(P \mid Q)|R \sim P|(Q \mid R)$

This result is not in contradiction with the one presented by Klin and Sassone [2008], where it is proved that associativity of parallel composition does not hold for CCS-like calculi if one uses PEPA-like minimal rate synchronization. Our result relies on the introduction of distinct labels for synchronization transitions $\left(\delta_{\widehat{a}}^{e}\right)$, which keep track of the interaction channel. This is necessary to properly compute renormalization while taking into account possible new inputs popping up along the derivation. The synchronization labels in [Klin and Sassone 2008] are just $\tau$; thus, crucial information is lost.

Notice that when deterministic FsFuTS with $\mathbb{R}_{\geq 0}$-valued continuations are considered (like in the case of the considered stochastic process calculi), strong Markovian bisimilarity, denoted by $\sim_{M}$, coincides with the bisimilarity relation $\sim$ of Definition 2.9 . We refer the readers interested in further details of Markovian bisimilarity to, e.g. [De Nicola et al. 2009c; Hillston 1996; Brinksma and Hermanns 2001].

The CTMC of the interactions within process $P \in \mathcal{P}_{\text {StoCCS }}$ can be derived as expected. The set of states is $\left(\mathcal{P}_{\text {StoCCS }}{ }_{A P}\right) / P$; the rate matrix is defined as follows, for each $Q_{1}, Q_{2} \in$ 
$\left(\mathcal{P}_{\left.\operatorname{StoCCS}_{A P}\right) / p:}\right.$

$$
\mathbf{R} Q_{1} Q_{2}=\sum_{\text {def }} \sum_{\mathscr{P} \in\left\{\mathscr{P}^{\prime} \mid \exists \alpha \in \Delta_{\widehat{\mathcal{P}}} \cdot Q_{1} \stackrel{\alpha}{\rightarrow} \mathscr{P}^{\prime}\right\}}\left(\mathscr{P} Q_{2}\right)
$$

In Appendix D.7 it is shown that the semantics we considered in Sect. 7.1 can be modified to obtain associativity of the CCS parallel composition, thus obtaining:

Theorem 7.9. For all $P, Q, R \in \mathcal{P}_{\text {StoCCS }_{A A}},(P \mid Q)|R \sim P|(Q \mid R)$

\section{INCLUDING NON-DETERMINISM AND PROBABILITIES}

In this section we address models where stochastically timed, non-deterministic and probabilistic behaviors coexist. We shall consider the Language of Interactive Markov Chains (IML) [Hermanns 2002] and Markov Automata [Eisentraut et al. 2010].

Before doing this, we introduce a general definition of FuTSs, which allows the transition relation to have continuations with different (semi-rings as) co-domains. For instance, continuations assigning rates to processes will be functions in $\mathbf{T F}\left(S, \mathbb{R}_{\geq 0}\right)$ for some state set $S$, as we have seen in the previous sections, while those assigning probabilities to processes will lay in $\mathbf{T F}(S,[\mathbf{0}, \mathbf{1}])$. Continuations used for expressing pure reachability, where one is not interested in quantitative issues, will be functions in $\operatorname{TF}(S, \mathbb{B})$; this way we can easily model pure non-deterministic behavior. In order to make different continuations coexist in the same FuTS, we use disjoint unions, denoted by $\uplus$, of the corresponding types. For instance, a FuTS where we want to represent both non-deterministic and probabilistic behavior will have a transition relation $\hookrightarrow \subseteq S \times A \times(\mathbf{T F}(S, \mathbb{B}) \uplus \mathbf{T F}(S,[\mathbf{0}, \mathbf{1}]))$, where boolean and probabilistic continuations coexist. The general definition of FuTSs follows:

Definition 8.1. A state to function A-labelled transition system (FuTS) over $\left\{\mathbb{C}_{j}\right\}_{j=1}^{k}$ is a tuple $\left(S, A,\left\{\mathbb{C}_{j}\right\}_{j=1}^{k}, \neg\right)$ where $S$ is a countable, non-empty, set of states, $A$ is a countable, non-empty, set of transition labels, $\left\{\mathbb{C}_{j}\right\}_{j=1}^{k}$ is a finite family of commutative semi-rings, and $\neg \subseteq S \times A \times \biguplus_{j=1}^{k} \mathbf{T F}\left(S, \mathbb{C}_{j}\right)$ is the transition relation.

As in the previous sections, FuTSs will be denoted by $\mathcal{R}, \mathcal{R}_{1}, \mathcal{R}^{\prime}, \ldots$. Furthermore, all notational conventions as well as definitions, e.g. total, deterministic, finite support FuTS, are extended in a natural way. In the following two sections we first consider Interactive Markov Chains (IMCs), where non-deterministic behavior is integrated with stochastically timed behavior, and then Markov Automata, which extend IMCs with probabilistic behavior. For each model we consider an appropriate language and provide its FUTS semantics.

\subsection{A Language for Interactive Markov Chains}

The key feature of Interactive Markov Chains (IMCs) [Hermanns 2002; Hermanns and Katoen 2010] is a definite, clear distinction between transitions modeling (instantaneous) actions, called interactive transitions, and transitions modeling the passage of time, called Markovian transitions because durations are, as usual, modeled by exponentially distributed random variables.

Definition 8.2. An Interactive Markov Chain (IMC) is a tuple $\left(S, A, \rightarrow,-\rightarrow, s_{0}\right)$ with $S$ a nonempty finite set of states, $A$ a finite set of actions, $\rightarrow \subseteq S \times A \times S$ the set of interactive transitions, $\rightarrow \subseteq S \times \mathbb{R}_{>0} \times S$ the set of Markov transitions, and $s_{0} \in S$ the initial state. - 
We could use simple FuTSs for IMCs, by just considering as co-domain of continuations a semi-ring of the form $\mathbb{R}_{\geq 0} \cup\{\infty\}$, where classical operations on reals are extended as follows: $\forall x \neq 0: x \cdot \infty=\infty, 0 \cdot \infty=0$ and $\forall x \cdot x+\infty=\infty$. Elements in $\mathbb{R}_{>0}$ would indicate rates of Markovian transitions (with 0 denoting un-reachability, as usual), while $\infty$ would characterize interactive ones, following the intuition that the latter, being immediate, have an infinite rate. This simple choice has, however, the disadvantage of obscuring the distinction between non-determinism and stochasticity. For this reason, we use general FuTSs with a transition relation whose continuation components are in $\mathbf{F T F}(S, \mathbb{B}) \uplus \mathbf{F T F}\left(S, \mathbb{R}_{\geq 0}\right)$. Continuations in $\operatorname{FTF}(S, \mathbb{B})$ are used to model non-deterministic transitions, while functions in $\mathbf{F T F}\left(S, \mathbb{R}_{\geq 0}\right)$ are used to describe stochastic behaviors.

Let us now focus on the FUTS semantics of the Language for IMCs (IML) proposed by Hermanns [2002]. As usual, we restrict our attention to a significative kernel of the full calculus; the relevant operators are: inaction, rate prefix, action prefix, choice, multi-party synchronization, and constant. Consequently, the set $\mathcal{P}_{I M L_{k}}$ of $\mathrm{IML}_{k}$ terms is defined by the grammar obtained by selecting from Fig. 2 the productions for the above mentioned operators. Note that, due to the distinction between actions and delays, $\mathrm{IML}_{k}$ has two different prefix operators, namely rate prefix, for delays, and action prefix for actions.

The relevant sets of states and labels are $\mathcal{P}_{I M L_{k}}$ and $\mathcal{L}_{I M L_{k}}={ }_{\text {def }} \mathcal{A} \cup\left\{\delta^{e}\right\}$, the latter including duration-less actions in $\mathcal{A}$ and the delay label $\delta^{e}$. Function $n: \mathcal{L}_{I M L_{k}} \rightarrow \mathcal{A} \cup\{\epsilon\}$ is defined by $n a=_{\text {def }} a$ and $n \delta^{e}=_{\text {def }} \epsilon$, assuming $\epsilon \notin \mathcal{A}$. As anticipated above, we use the semi-rings $\mathbb{B}$ and $\mathbb{R}_{\geq 0}$. The set of rules defining the transition relation $r$ is composed of rules (CHO) and (CNS) of Fig. 3 (where $P, Q \in \mathcal{P}_{I M L_{k}}$ and $\alpha \in \mathcal{L}_{I M L_{k}}$ is assumed) and of the rules of Fig. 14, where, with a bit of overloading, we refine functions $\mathcal{X}$ and $\otimes_{\times}$as described below. We let function $\mathcal{X}: \mathcal{L}_{I M L_{k}} \rightarrow \mathcal{P}_{I M L_{k}} \rightarrow\left(\mathbf{T F}\left(\mathcal{P}_{I M L_{k}}, \mathbb{B}\right) \uplus \mathbf{T F}\left(\mathcal{P}_{I M L_{k}}, \mathbb{R}_{\geq 0}\right)\right)$ be defined as follows:

$$
\mathcal{X}_{\alpha}=_{\operatorname{def}} \begin{cases}\mathcal{X}_{\mathbb{B}}, & \text { if } \alpha \in \mathcal{A}, \\ \mathcal{X}_{\mathbb{R}_{20}}, & \text { if } \alpha=\delta^{e} .\end{cases}
$$

Let, furthermore, $\mathbf{B O P}(F)=_{\text {def }} F \times F \rightarrow F$ denote the collection of all the binary operations on $F$. We let function $\otimes_{\|_{L}}: \mathcal{L}_{I M L_{k}} \rightarrow \mathbf{B O P}\left(\mathbf{T F}\left(\mathcal{P}_{I M L_{k}}, \mathbb{B}\right)\right) \uplus \mathbf{B O P}\left(\mathbf{T F}\left(\mathcal{P}_{I M L_{k}}, \mathbb{R}_{\geq 0}\right)\right)$ be defined as follows:

$$
\otimes_{\|_{L}}^{\alpha}==_{\text {def }} \begin{cases}\otimes_{\|_{L}}^{\mathbb{B}}, \text { if } \alpha \in \mathcal{A}, \\ \otimes_{\|_{L}}^{\mathbb{R}_{\geq 0}}, \text { if } \alpha=\delta^{e} .\end{cases}
$$

Notably, operators $\mathcal{X} \alpha$ and $\otimes_{\|_{L}}^{\alpha}$ are used to avoid type mismatches in the continuation formulas while guaranteeing that the same rules for interleaving (and, similarly, for) synchronization are used, regardless of the type of continuation functions. In fact, the semantic rules for $\mathrm{IML}_{k}$ are the same as those for $\mathrm{TIPP}_{k}$ except for parameter $\alpha$ in the functions above. Actually, one could use these generalized functions also in the definitions of $\operatorname{TIPP}_{k}$ and of all the other SPCs. Then the same rule format, and in the case of $\mathrm{IML}_{k}$ and $\mathrm{TIPP}_{k}$ exactly the same rules, could be used for all the calculi. We preferred to use different formats for the sake of readability.

The following proposition can be easily proven by derivation induction:

Proposition 8.3. For all $P \in \mathcal{P}_{I M L_{k}}, \alpha \in \mathcal{L}_{I M L_{k}}$, and $\mathscr{P} \in \mathbf{T F}\left(\mathcal{P}_{I M L_{k}}, \mathbb{R}_{\geq 0} \uplus \mathbb{B}\right)$, if $P \stackrel{\alpha}{\rightarrow} \mathscr{P}$ can be derived using the set consisting of rules $(C H O)$ and (CNS) of Fig. 3 and of the rules of Fig. 14, then the following holds: $\mathscr{P} \in \mathbf{F T F}\left(\mathcal{P}_{I M L_{k}}, \mathbb{R}_{\geq 0} \uplus \mathbb{B}\right)$. 


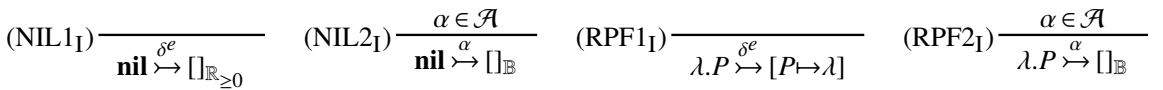

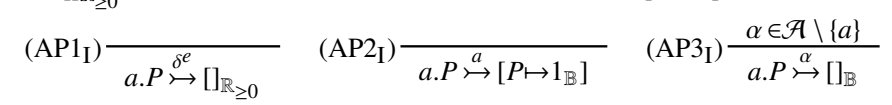

$$
\begin{aligned}
& \left(\mathrm{PAR}_{\mathrm{I}}\right) \frac{P \stackrel{\alpha}{\mapsto} \mathscr{P}, Q \stackrel{\alpha}{\mapsto} \mathscr{Q},(n \alpha) \notin L}{P \|_{L} Q \stackrel{\alpha}{\mapsto}\left(\mathscr{P} \otimes_{\|_{L}}^{\alpha}(\mathcal{X} \alpha Q)\right)+\left((X \alpha P) \otimes_{\|_{L}}^{\alpha} \mathscr{Q}\right)} \quad\left(\mathrm{PAR} 2_{\mathrm{I}}\right) \frac{P \stackrel{\alpha}{\mapsto} \mathscr{P}, Q \stackrel{\alpha}{\mapsto} \mathscr{Q},(n \alpha) \in L}{P \|_{L} Q \stackrel{\alpha}{\mapsto} \mathscr{P} \otimes_{\|_{L}}^{\alpha} \mathscr{Q}}
\end{aligned}
$$

Fig. 14. Additional semantic rules for $\mathrm{IML}_{k}$

Proposition 8.4. For all $P \in \mathcal{P}_{I M L_{k}}, \alpha \in \mathcal{L}_{I M L_{k}}$ and $\mathscr{P} \in \mathbf{F T F}\left(\mathcal{P}_{I M L_{k}}, \mathbb{R}_{\geq 0} \uplus \mathbb{B}\right)$ such that $P \stackrel{\alpha}{\longmapsto} \mathscr{P}$ can be derived using the set consisting of rules $(\mathrm{CHO})$ and $(\mathrm{CNS})$ of Fig. 3 and of the rules of Fig. 14, the following holds: (i) if $\alpha \in \mathcal{A}$ then $\mathscr{P} \in \mathbf{F T F}\left(\mathcal{P}_{I M L_{k}}, \mathbb{B}\right) ;($ ii) if $\alpha=\delta^{e}$ then $\mathscr{P} \in \mathbf{F T F}\left(\mathcal{P}_{I M L_{k}}, \mathbb{R}_{\geq 0}\right)$.

Definition 8.5. The formal semantics of $I M L_{k}$ is the FsFuTS $\mathcal{R}_{I M L_{k}}$ defined as the tuple $\left(\mathcal{P}_{I M L_{k}}, \mathcal{L}_{I M L_{k}},\left\{\mathbb{R}_{\geq 0}, \mathbb{B}\right\}, \mapsto\right)$ where $\mapsto \subseteq \mathcal{P}_{I M L_{k}} \times \mathcal{L}_{I M L_{k}} \times\left(\mathbf{F T F}\left(\mathcal{P}_{I M L_{k}}, \mathbb{B}\right) \biguplus \mathbf{F T F}\left(\mathcal{P}_{I M L_{k}}, \mathbb{R}_{\geq 0}\right)\right)$ is the least relation satisfying the set consisting of rules (CHO) and (CNS) of Fig. 3 and of the rules of Fig. 14.

The following theorem characterizes the structure of $\mathcal{R}_{I M L_{k}}$.

THEOREM 8.6. $\mathcal{R}_{I M L_{k}}$ is total and deterministic.

The following theorem establishes the formal correspondence between the FUTS of $\mathrm{IML}_{k}$ and the semantics definition given in [Hermanns 2002]. As usual, we let the cumulative transition rate from $P_{1}$ to $P_{2}$ be denoted by $\operatorname{rt}\left(P_{1}, P_{2}\right)$.

Theorem 8.7. For all $P, Q \in \mathcal{P}_{I M L_{k}}, a \in \mathcal{A}$, and unique functions $\mathscr{P} \in \mathbf{F T F}\left(\mathcal{P}_{I M L_{k}}, \mathbb{B}\right)$ and $\mathscr{P}^{\prime} \in \mathbf{F T F}\left(\mathcal{P}_{I M L_{k}}, \mathbb{R}_{\geq 0}\right)$ such that $P \stackrel{a}{\longmapsto} \mathscr{P}$ and $P \stackrel{\delta^{e}}{\longmapsto} \mathscr{P}^{\prime}$ the following holds: (i) $(\mathscr{P} Q)=1_{\mathbb{B}}$ if and only if $P \stackrel{a}{\rightarrow} Q ;($ ii $)\left(\mathscr{P}^{\prime} Q\right)=\mathbf{r t}(P, Q)$.

\subsection{A Language for Markov Automata}

Markov Automata (MAs) [Eisentraut et al. 2010] are an extension of IMCs where probabilistic behavior is integrated with non-deterministic and stochastically timed behavior. Essentially, IMCs interactive transitions are replaced by probabilistic transitions in MAs. Any probabilistic transition is labelled by an action, just like IMCs interactive transitions; however, instead of pointing to a single process, it points to a process sub-distribution. MAs generalize IMCs in the sense that IMCs are isomorphic to MAs where all sub-distributions are the unit Dirac distribution. Similarly, MAs generalize Probabilistic Automata of Segala [1995] by introducing a notion of global (stochastic) time. MAs are defined below ${ }^{8}$ we let Distr(S) denote the collections of all probability sub-distributions over $S$ with finite support, $\operatorname{Distr}(\mathrm{S})=_{\text {def }}\left\{\mathscr{P} \in \mathbf{F T F}\left(S, \mathbb{R}_{\geq 0}\right) \mid \oplus \mathscr{P} \leq 1\right\}$.

Definition 8.8. A Markov Automaton (MA) is a tuple $\left(S, A, \rightarrow, \rightarrow \rightarrow, s_{0}\right)$ where $S$ is a nonempty finite set of states, $A$ a finite set of actions, $\rightarrow \subseteq S \times A \times \operatorname{Distr}(\mathrm{S})$ is the set of probabilistic transitions, $\rightarrow \subseteq S \times \mathbb{R}_{>0} \times S$ is the set of Markov timed transitions, and $s_{0} \in S$ the initial state.

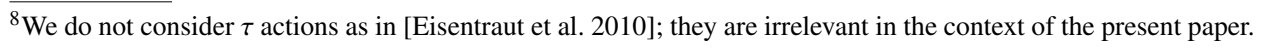
ACM Computing Surveys - In Press, Vol. , No. , November 2012. 


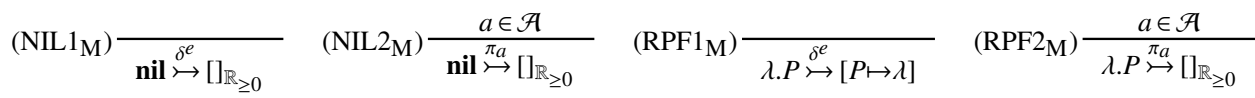

$$
\begin{aligned}
& \left(\mathrm{PAP}_{\mathrm{M}}\right) \frac{}{a .\left\{\sum_{h} p_{h}:: P_{h}\right\} \stackrel{\delta^{e}}{\mapsto}[]_{\mathbb{R}_{\geq 0}}} \\
& \left(\mathrm{PAP}_{\mathrm{M}}\right) \frac{b \in \mathcal{A} \backslash\{a\}}{a .\left\{\sum_{h} p_{h}:: P_{h}\right\} \stackrel{\pi_{a}}{\rightarrow} \mathbb{L}\left\{\sum_{h} p_{h}:: P_{h}\right\} \rrbracket} \quad\left(\mathrm{PAP}_{\mathrm{M}}\right) \frac{\stackrel{\pi_{b}}{\mapsto}[]_{\mathbb{R}_{\geq 0}}}{a \cdot\left\{\sum_{h} p_{h}:: P_{h}\right.} \\
& \left(\mathrm{CHO}_{\mathrm{M}}\right) \frac{P \stackrel{\delta^{e}}{\mapsto} \mathscr{P}, Q \stackrel{\delta^{e}}{\mapsto} \mathscr{Q}}{P+Q \stackrel{\delta^{e}}{\mapsto} \mathscr{P}+\mathscr{Q}} \quad\left(\mathrm{CHO}_{\mathrm{M}}\right) \frac{P \stackrel{\pi_{a}}{\mapsto} \mathscr{P}}{P+Q \stackrel{\pi_{a}}{\rightarrow} \mathscr{P}} \quad\left(\mathrm{CHO}_{\mathrm{M}}\right) \frac{Q \stackrel{\pi_{a}}{\mapsto} \mathscr{Q}}{P+Q \stackrel{\pi_{a}}{\mapsto} \mathscr{Q}} \\
& \left(\mathrm{PAR} 1_{\mathrm{M}}\right) \frac{P \stackrel{\delta^{e}}{\mapsto} \mathscr{P}, Q \stackrel{\delta^{e}}{\mapsto} \mathscr{Q}}{P \|_{L} Q \stackrel{\delta^{e}}{\longmapsto}\left(\mathscr{P} \otimes_{\|_{L}}(X Q)\right)+\left((X P) \otimes_{\|_{L}} \mathscr{Q}\right)} \\
& \left(\mathrm{PAR} 2_{\mathrm{M}}\right) \frac{P \stackrel{\pi_{a}}{\rightarrow} \mathscr{P}, a \notin L}{P \|_{L} Q \stackrel{\pi_{a}}{\rightarrow} \mathscr{P} \otimes_{\|_{L}}(X Q)} \quad\left(\mathrm{PAR} 3_{\mathrm{M}}\right) \frac{Q \stackrel{\pi_{a}}{\rightarrow} \mathscr{Q}, a \notin L}{P \|_{L} Q \stackrel{\pi_{a}}{\rightarrow}(X P) \otimes_{\|_{L}} \mathscr{Q}} \\
& (\mathrm{PAR} 4) \frac{P \stackrel{\pi_{a}}{\rightarrow} \mathscr{P}, Q \stackrel{\pi_{a}}{\rightarrow} \mathscr{Q}, a \in L}{P \|_{L} Q \stackrel{\pi_{a}}{\rightarrow} \mathscr{P} \otimes_{\|_{L}} \mathscr{Q}}
\end{aligned}
$$

Fig. 15. Additional semantic rules for MAL

Recently, a full language for Markov Automata, MAPA (Markov Automata Process Algebra) has been proposed by Timmer et al. [2012]. MAPA includes also a rich data system and is equipped with restrictions for enabling state space generation and equivalent smaller models. In [Timmer et al. 2012], the semantics of MAPA is defined using the SOS style. In particular, proved LTSs are used for the markovian part of the language. Below, we introduce MAL, a simplified fragment of MAPA, which we use for showing how MAs based languages can be modeled with FUTSs. The constructs of MAL are: inaction, rate prefix, probabilistic action prefix, choice, multi-party synchronization, and constant. Consequently, the set $\mathcal{P}_{M A L}$ of MAL terms is defined by the grammar obtained by selecting from Fig. 2 the productions for the above mentioned constructs. Notice that the only difference from $\mathrm{IML}_{k}$ is that action prefix has been replaced by probabilistic action prefix. The notation $\left\{p_{1}:: P_{1}+\ldots+p_{h}:: P_{h}\right\}$ in probabilistic action prefix uniquely characterizes the probability sub-distribution $\left[P_{1} \mapsto p_{1} \ldots P_{h} \mapsto p_{h}\right] \in \operatorname{DisTR}\left(\mathcal{P}_{\text {MAL }}\right)$, which we denote by $\llbracket\left\{p_{1}:: P_{1}+\ldots+p_{h}:: P_{h}\right\} \rrbracket$. The relevant set of states is $\mathcal{P}_{M A L}$. For what concerns the label set, recall that we conventionally let $\pi$ label probabilistic transitions (see Remark 2.8). Since in MAs such transitions are associated with actions in the action set $\mathcal{A}$, the label set must include the set $\Pi_{\mathcal{A}}$, defined as $\left\{\pi_{a} \mid a \in \mathcal{A}\right\}$; furthermore, it must include $\delta^{e}$, since MAL models also delays. Thus we define $\mathcal{L}_{M A L}==_{\text {def }} \Pi_{\mathcal{A}} \cup\left\{\delta^{e}\right\}$, with $\delta^{e} \notin \Pi_{\mathcal{A}}$. Function $n: \mathcal{L}_{M A L} \rightarrow \mathcal{A} \cup\{\epsilon\}$ is defined by $n \pi_{a}={ }_{\text {def }} a$ and $n \delta^{e}=_{\text {def }} \epsilon$, assuming $\epsilon \notin \mathcal{A}$. The relevant semi-ring is $\mathbb{R}_{\geq 0}$. Furthermore, we have that if $P \stackrel{\pi_{a}}{\longmapsto} \mathscr{P}$, then $\oplus \mathscr{P} \leq 1$. We will use functions $\mathcal{X}_{\mathbb{R}_{\geq 0}}$ and $\otimes_{\|_{L}}^{\mathbb{R}_{\geq 0}}$, as introduced in Section 5.2 on page 15. The set of rules defining the transition relation is given in Fig. 15 plus Rule (CNS) of Fig. 3, where $P, Q \in \mathcal{P}_{M A L}$ and $\alpha \in \mathcal{L}_{M A L}$ is assumed. It is worth noting that the rules for the fragment of MAL dealing with exponentially distributed delays, namely Rules $\left(\mathrm{NIL} 1_{\mathrm{M}}\right),\left(\mathrm{RPF} 1_{\mathrm{M}}\right),\left(\mathrm{CHO} 1_{\mathrm{M}}\right)$ and $\left(\mathrm{PAR} 1_{\mathrm{M}}\right)$ are the same as those for $\mathrm{IML}_{k}$. The other rules deal with probability sub-distributions and non-determinism at once. The following propositions can be easily proven by derivation induction: 
Proposition 8.9. For all $P \in \mathcal{P}_{M A L}, \alpha \in \mathcal{L}_{M A L}$, and $\mathscr{P} \in \mathbf{T F}\left(\mathcal{P}_{M A L}, \mathbb{R}_{\geq 0}\right)$, if $P \stackrel{\alpha}{\longmapsto} \mathscr{P}$ can be derived using the set consisting of rules (CNS) of Fig. 3 and of the rules of Fig. 15, then the following holds: $\mathscr{P} \in \mathbf{F T F}\left(\mathcal{P}_{M A L}, \mathbb{R}_{\geq 0}\right)$.

Proposition 8.10. For all $P \in \mathcal{P}_{M A L}, \alpha \in \mathcal{L}_{M A L}$ and $\mathscr{P} \in \mathbf{F T F}\left(\mathcal{P}_{M A L}, \mathbb{R}_{\geq 0}\right)$ such that $P \stackrel{\alpha}{\longmapsto} \mathscr{P}$ can be derived using the set consisting of rules (CNS) of Fig. 3 and of the rules of Fig. 15, if $\alpha \in \Pi_{\mathcal{A}}$ then $\oplus \mathscr{P} \leq 1$.

Definition 8.11. The formal semantics of $M A L$ is the FsFuTS $\mathcal{R}_{M A L}$ defined as the tuple $\left(\mathcal{P}_{M A L}, \mathcal{L}_{M A L}, \mathbb{R}_{\geq 0}, \mapsto\right) ; \neg \subseteq \mathcal{P}_{M A L} \times \mathcal{L}_{M A L} \times \mathbf{F T F}\left(\mathcal{P}_{M A L}, \mathbb{R}_{\geq 0}\right)$ is the least relation satisfying the set consisting of rules (CNS) of Fig. 3 and of the rules of Fig. 15.

The following theorem characterizes the structure of $\mathcal{R}_{M A L}$.

THEOREM 8.12. $\mathcal{R}_{\text {MAL }}$ is total.

It is worth noting that $\mathcal{R}_{M A L}$ is not deterministic. This is due to the way we decided to deal with probabilistic transitions. In $\mathrm{IML}_{k}$ we represented non-determinism using boolean functions. For instance, a term like $a . P_{1}+a . P_{2}$, with $P_{1} \neq P_{2}$ and $a \in \mathcal{A}$, gives rise to the following transition:

$$
a . P_{1}+a . P_{2} \stackrel{a}{\longmapsto}\left[P_{1} \mapsto \text { TRUE, } P_{2} \mapsto \text { TRUE }\right]
$$

Boolean functions are a compact way of representing non-determinism in the choice of the next process ( $P_{1}$ or $P_{2}$ in the example above). We could have used a similar approach for non-deterministic choice over process (sub-)distributions. For instance a term like $a .\left\{\sum_{i} p_{i}:: P_{i}\right\}+a .\left\{\sum_{j} q_{j}:: Q_{j}\right\}$, with $\left\{\sum_{i} p_{i}:: P_{i}\right\} \neq\left\{\sum_{j} q_{j}:: Q_{j}\right\}$ and $a \in \mathcal{A}$ would have given rise to the following transition:

$a .\left\{\sum_{i} p_{i}:: P_{i}\right\}+a .\left\{\sum_{j} q_{j}:: Q_{j}\right\} \stackrel{\pi_{a}}{\mapsto}\left[\llbracket\left\{\sum_{i} p_{i}:: P_{i}\right\} \rrbracket \mapsto \mathrm{TRUE},\left[\left\{\sum_{j} q_{j}:: Q_{j}\right\} \rrbracket \mapsto \mathrm{TRUE}\right]\right.$

This solution would have required MAL probabilistic continuation functions be of type TF(Distr(MAL), $\mathbb{B}$ ), thus breaking the property of the FuTS framework presented in this paper, namely that continuations are of type $\operatorname{TF}\left(\mathcal{P}_{C}, \mathbb{C}\right)$ for some commutative semi-ring $\mathbb{C}$, where $\mathcal{P}_{C}$ is always a set of syntactical terms. Consequently, under the semantics given in Definition 8.11, the above term gives rise to the following two transitions:

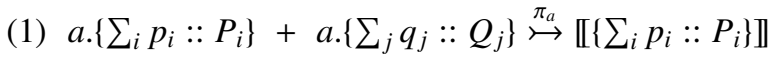

(2) $a .\left\{\sum_{i} p_{i}:: P_{i}\right\}+a .\left\{\sum_{j} q_{j}:: Q_{j}\right\} \stackrel{\pi_{a}}{\rightarrow} \llbracket\left\{\sum_{j} q_{j}:: Q_{j}\right\} \rrbracket$.

\section{RELATED WORK}

Our approach is mainly based on three notions: the use of functions in the transition relation, the generalization of the co-domain of such functions to generic commutative semi-rings, and a systematic use of composition operators for such functions, which facilitates a compositional, syntax-driven definition of the semantics of SPCs. The use of functions in the transitions is not new; for instance they can be found also in [Deng et al. 2007] and [Bohnenkamp et al. 2006]. However, in both works, function codomains are not generic. Deng et al. [2007] also employ a systematic usage of composition operators on functions.

ACM Computing Surveys - In Press, Vol. , No., November 2012. 
Other work aiming at providing a systematic account of the semantics of stochastic calculi has been presented in [Klin and Sassone 2008] that studies a (meta-)syntactic framework, called SGSOS, for defining well-behaved Markovian stochastic transition systems.

The SGSOS format arises from the abstract theory of well-behaved operational semantics, based on bialgebras and a type of natural transformations, namely distributive laws. SGSOS specifications induce Rated TSs, which are very similar to RTSs [De Nicola et al. 2009 c], i.e. a sub-class of FuTSs. Rates of transitions in the Rated TSs induced by a SGSOS specification, are computed by induction on the syntax of process terms and by taking into account the contribution of all those SGSOS rules that are triggered by the relevant (apparent) rates. Note that such a set of rules is finite. So, in a sense, the computation of the rates is distributed among the (instantiations of the) relevant rules with intermediate results collected (and summed up) in the final rate. In this sense, the SGSOS approach is more 'syntax-oriented' than the RTS one. In the latter, the relevant values are manipulated in a more direct way, using the operators on continuation functions, by applying them directly to the continuation elements of the transitions within the semantics rules. A noteworthy result of Klin and Sassone [2008] is that stochastic bisimilarity of SPCs defined using the SGSOS format is guaranteed to be a congruence. This result is generalized by Klin [2009] who studies Weighted Transition Systems (WTSs) and related Weighted GSOS which are proven to induce a congruence. It is shown that Rated TSs as well as LTSs are special cases of WTSs. The above considerations on the comparison between the SGSOS and RTSs approaches apply also to Weighted GSOS and general total deterministic FsFuTSs.

The approach taken recently in [Cardelli and Mardare 2010], where the semantics of stochastic calculi is defined by associating a measure with each term, which encodes the rates of the transitions from the state of a system to a measurable set of states, is somehow opposite in spirit to ours. They associate measures with sets of processes while we exploit functions from processes to semi-rings for building operational models. It has to be said, however, that their semantic definitions rely heavily on general functions on measure spaces and on operators which are very similar to those we proposed in [De Nicola et al. 2009a; 2009c] and that we have been using here. In [Cardelli and Mardare 2010] it is stated that their approach generalizes ours because they deal with measures. On the other hand, our focus is on the way continuation functions are used, manipulated and generalized (by using generic semi-rings) in order to deal with very different models, ranging from CTMCs to DTMCs as well as combinations thereof. To the best of our knowledge both Klin and Sassone [2008] and Cardelli and Mardare [2010] have only considered fully stochastic (i.e. without non-determinism) calculi, with one-to-one synchronization.

Hojjat et al. [2008] also aim at providing a uniform account of the semantics of different SPCs with the main objective of automatic analysis of stochastic processes. They take an axiomatic approach and use axioms as rewriting rules to reduce process terms into the common format used by the $\mu$ CRL toolset [Blom et al. 2001].

In [Latella et al. 2012] the notion of bisimulation induced by FuTSs is addressed from a co-algebraic perspective. A correspondence result is proven stating that FUTS-bisimulation coincides with the behavioral equivalence of the associated functor. As concrete examples, the original equivalences for $\mathrm{PEPA}_{k}$ and $\mathrm{IML}_{k}$ are related to FuTS-bisimulation, providing a coalgebraic justification for these equivalences.

Bernardo et al. [2010] proposed Ultras, a model similar to FuTSs, in order to capture and study non-deterministic, probabilistic, and stochastic trace and bisimulation equiva- 
lences. In Ultras, function co-domains are not required to be commutative semi-rings, but rather they are order sets with a bottom element denoting unreachability.

We would like to conclude by mentioning only a few other models that are related to FuTSs. The structure of Continuous Time Markov Decision Processes (CTMDPs), as defined by Hermanns and Johr [Hermanns and Johr 2007], is similar to the structure of finite support FuTSs with action-indexed random delays $\left(\Delta_{\mathcal{A}}\right.$-labeled FsFuTS over $\left.\mathbb{R}_{\geq 0}\right)$. Indeed, a CTMDP is a tuple $\mathcal{M}=\left(S, \mathcal{A}, T, s_{0}\right)$ with $S$ a (finite) set of states, $\mathcal{A}$ a (finite) set of action labels, $s_{0} \in S$, and $T \subseteq S \times \mathcal{A} \times \mathbf{F T F}\left(S, \mathbb{R}_{\geq 0}\right)$ the transition relation. Despite the strong structural similarity, there are, however, important conceptual differences between the two models. In fact, while in $\Delta_{\mathcal{A}}$-labeled FsFuTSs the action to perform is selected among those enabled following the race condition principle, in CTMDPs, choices are based on a reactive semantics. Thus the next action is selected by the environment, while the race condition principle is used to select the next state. It is not difficult to see that CTMDPs can be defined as FuTSs with the appropriate choice of transition labels and appropriate co-domains of the continuation functions. The same applies to other models proposed in the literature under the name Continuous Time Probabilistic Automata [Lijun Zhang et al. 2008; Knast 1969; Dang Van Hung and Zhou Chaochen 1999]. All these variants can be rendered as FuTSs. Indeed, for the model proposed in [Lijun Zhang et al. 2008] similar considerations as those for CTMDPs apply. The Continuous Time Probabilistic Automata considered in [Knast 1969] have been proposed as a language theoretic framework; the element $a_{i, j}(x)$ of the infinitesimal matrix used there is modeled, in our approach, with $(\mathscr{P} j)$

for $i \stackrel{\delta_{x}^{e}}{\rightarrow} \mathscr{P}$. Finally, the variant used in [Dang Van Hung and Zhou Chaochen 1999] is based on standard automata, where transitions are elements of $S \times S$ and have a rate but no label associated. Thus they are directly related to $\left\{\delta^{e}\right\}$-labelled FuTSs over $\mathbb{R}_{\geq 0}$.

\section{CONCLUSIONS}

In this article we have presented a number of stochastic process calculi and have defined their structural operational semantics relying on the same semantic framework based on so-called State to Function Labeled Transition Systems (FuTSs) that has facilitated the compact and compositional definition of the different semantics.

The key feature of FuTSs is the fact that each transition is a triple of the form $(s, \alpha, \mathscr{P})$. The first and the second components are the source state and the label of the transition, while the third component, $\mathscr{P}$, is the continuation function, which associates a value of a suitable type with each state, say $s^{\prime}$. The only requirement on the co-domains of the continuation functions is that they must be commutative semi-rings, which make FuTSs a very general framework. This provides a high level of flexibility while preserving basic properties of primitive operations like sum and multiplication. Moreover, since the third component of the transition relation can be also a disjoint union of sets of functions with different co-domains, FuTSs can be used to model different 'kinds' of transitions by associating different co-domains to continuations. Indeed, in this paper we have shown that FuTSs can be effectively used as a semantic domain for the compositional definition of the operational semantics of a calculus with both non-deterministic behavior and stochastic delays, and for an extension including probabilistic discrete (sub-)distributions over processes.

By defining appropriate operators on continuation functions, we have provided a compositional operational semantics of key fragments of major stochastic process calculi in- 
cluding TIPP, EMPA, PEPA, StoCCS, IML and MAL, a language for Markov Automata. By this, we have provided a uniform, clean and powerful framework which supports the identification of differences and similarities.

FuTSs elegantly solve the issue of transition multiplicity; the rates of equal transitions, among those derivable from the semantics rules, are simply added via operations on continuation functions. Furthermore, FuTSs make it relatively easy to define associative parallel composition operators for calculi adopting the one-to-one interaction paradigm. Indeed, by appropriately defining the composition of continuation functions, the components to be taken into account, when one is interested in guaranteeing associativity of parallel composition, can be singled out and appropriately combined. Moreover, the unified framework clearly shows that the modeling of one-to-one synchronization becomes simpler when one distinguishes between active and passive actions; if all actions are considered as active the arithmetics of rates becomes much more intricate.

The correspondence result shown in [Latella et al. 2012] sets the basis for a systematic study of SPCs, within the FuTS framework, based on category theory, and in particular within the coalgebraic framework, similar to what has been done for probabilistic models in [Bartels 2002; Bartels et al. 2003; Sokolova and de Vink 2004], for Rated TSs by Klin and Sassone [2008] and WTSs [Klin 2009]. We think this is a promising line of future research which should be undertaken for FuTSs; in particular, the relationship between WTSs Weighted GSOS on one hand and FuTSs and related PCs sematics rules on the other seems an interesting line of research.

\section{ACKNOWLEDGMENTS}

We are very grateful to Marco Bernardo, Pedro D'Argenio, Gethin Norman, Bartek Klin, Roberto Segala, and Erik de Vink for valuable discussions and suggestions. We also thank the anonymous reviewers for their detailed and helpful reports. 


\section{APPENDIX: Detailed proofs for the electronic editions}

\section{A. SOS DEFINITIONS}

In this section the original SOS definition of the relevant process calculi is given. We warn the reader that in this and the following sections, we sometimes used a syntactic presentation which is different from the one of the original definition of the calculi at hand. The reason for that is uniformity of presentation. We furthermore want to point out that in the literature the transition multi-relation has often be defined as the least multi-relation satisfying a set of SOS rules (see, e.g. [Hillston 1996] or [Hermanns 2002]). Although this definition is not completely correct, since the least multi-relation happens to be a relation, thus not capturing, as a matter of fact, transition multiplicity, in the sequel we stick to the original formulation for conformance with the original proposals.

\section{A.1 Calculus for finite CTMCs}

The SOS of the Calculus for finite CTMCs of Sect. 4 is the multi-LTS $\left(\mathcal{P}_{C T M C}, \mathbb{R}_{>0}, \rightarrow\right)$ where $\rightarrow$ is the multi-relation induced by the rules given in Fig. 16 .

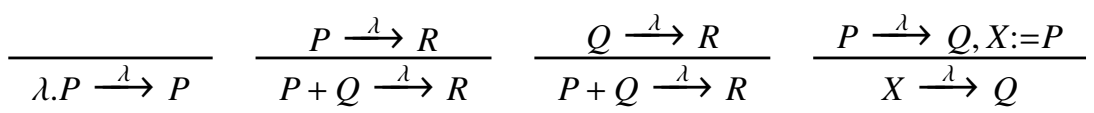

Fig. 16. SOS Rules for the CTMC Calculus.

\section{A.2 TIPP $_{k}$}

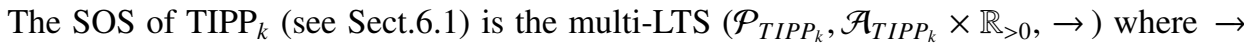
is the least multi-relation satisfying the rules given in Fig. 17. Notice that in the original definition of TIPP [Hermanns et al. 1998], the rated-action prefix, choice and parallel composition operators are denoted by $(a, \lambda) . P,[]$ and $|[L]|$ respectively.

$$
\begin{aligned}
& \underset{\langle a, \lambda\rangle . P \stackrel{a, \lambda}{\longrightarrow} P}{\frac{P \stackrel{a, \lambda}{\longrightarrow} R}{P+Q \stackrel{a, \lambda}{\longrightarrow} R}} \quad \frac{Q \stackrel{a, \lambda}{\longrightarrow} R}{P+Q \stackrel{a, \lambda}{\longrightarrow} R} \quad \frac{P \stackrel{a, \lambda}{\longrightarrow} Q, X:=P}{X \stackrel{a, \lambda}{\longrightarrow} Q} \\
& \frac{P \stackrel{a, \lambda}{\longrightarrow} P^{\prime}, a \notin L}{P\left\|_{L} Q \stackrel{a, \lambda}{\longrightarrow} P^{\prime}\right\|_{L} Q} \quad \frac{Q \stackrel{a, \lambda}{\longrightarrow} Q^{\prime}, a \notin L}{P\left\|_{L} Q \stackrel{a, \lambda}{\longrightarrow} P\right\|_{L} Q^{\prime}} \\
& \frac{P \stackrel{a, \lambda_{P}}{\longrightarrow} P^{\prime}, Q \stackrel{a, \lambda_{Q}}{\longrightarrow} Q^{\prime}, a \in L}{P\left\|_{L} Q \stackrel{a, \lambda_{P} \cdot \lambda_{Q}}{\longrightarrow} P^{\prime}\right\|_{L} Q^{\prime}}
\end{aligned}
$$

Fig. 17. SOS Rules for the $\operatorname{TIPP}_{k}$. 


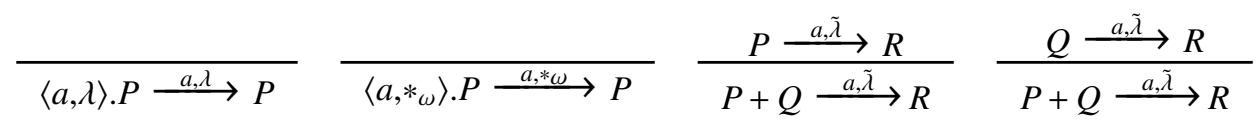

$$
\begin{aligned}
& \frac{P \stackrel{a, \tilde{\lambda}}{\longrightarrow} Q, X:=P}{X \stackrel{a, \tilde{\lambda}}{\longrightarrow} Q} \quad \frac{P \stackrel{a, \tilde{\lambda}}{\longrightarrow} P^{\prime}, a \notin L}{P\left\|_{L} Q \stackrel{a, \tilde{\lambda}}{\longrightarrow} P^{\prime}\right\|_{L} Q} \quad \frac{Q \stackrel{a, \tilde{\lambda}}{\longrightarrow} Q^{\prime}, a \notin L}{P\left\|_{L} Q \stackrel{a, \tilde{\lambda}}{\longrightarrow} P\right\|_{L} Q^{\prime}}
\end{aligned}
$$

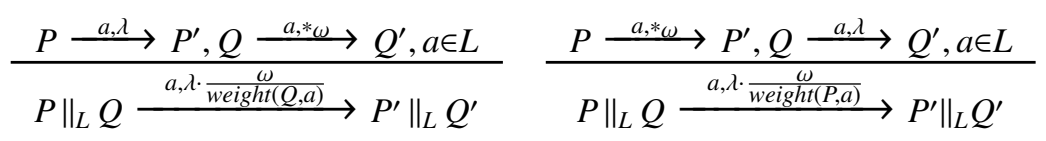

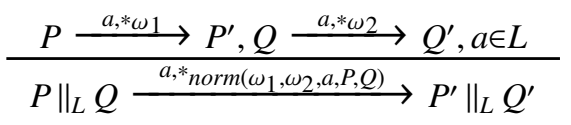

Fig. 18. SOS Rules for the $\mathrm{EMPA}_{k}$.

\section{A.3 $\mathrm{EMPA}_{k}$}

The SOS of $\mathrm{EMPA}_{k}$ (see Sect.6.2) is the multi-LTS $\left(\mathcal{P}_{E M P A_{k}}, \mathcal{A}_{E M P A_{k}} \times\left(\mathbb{R}_{>0} \cup\left\{*_{\omega} \mid \omega \in\right.\right.\right.$ $\left.\left.\mathbb{R}_{>0}\right\}\right), \rightarrow$ ) where $\rightarrow$ is the multi-relation induced by the rules given in Fig. 18. In the figure, $\tilde{\lambda} \in \mathbb{R}_{>0} \cup\left\{*_{\omega} \mid \omega \in \mathbb{R}_{>0}\right\}$, whereas weight $(P, a)$ and $\operatorname{norm}\left(\omega_{1}, \omega_{2}, a, P_{1}, P 2\right)$ are defined as follows, where $\sum\{\}=$ def 0 :

$$
\begin{aligned}
& \operatorname{weight}(P, a)==_{\operatorname{def}} \sum\left\{\omega \in \mathbb{R}_{>0} \mid \exists P^{\prime} \in \mathcal{P}_{E M P A_{k}} . P \stackrel{a, *_{\omega}}{\longrightarrow} P^{\prime}\right\} \\
& \operatorname{norm}\left(\omega_{1}, \omega_{2}, a, P_{1}, P 2\right)={ }_{\mathrm{def}} \\
& \frac{\omega_{1}}{\operatorname{weight}\left(P_{1}, a\right)} \cdot \frac{\omega_{2}}{\operatorname{weight}\left(P_{2}, a\right)} \cdot\left(\operatorname{weight}\left(P_{1}, a\right)+\operatorname{weight}\left(P_{2}, a\right)\right)
\end{aligned}
$$

In the original definition of EMPA [Aldini et al. 2010], the rec operator for constant definition is used, instead of defining equations.

\section{A.4 PEPA ${ }_{k}$}

The SOS of PEPA $A_{k}$ (see Sect.6.3) is the multi-LTS $\left(\mathcal{P}_{P E P A_{k}}, \mathcal{A}_{P E P A_{k}} \times \mathbb{R}_{>0}, \rightarrow\right)$ where $\rightarrow$ is the least multi-relation satisfying the rules given in Fig. 19. In the figure $r_{\alpha}(P)$ and $r\left(\alpha, \lambda_{1}, \lambda_{2}, P, Q\right)$ are used, which are defined as follows:

$$
\begin{aligned}
& r_{\alpha}((\beta, \lambda) . P)=_{\operatorname{def}}\left\{\begin{array}{l}
\lambda, \text { if } \beta=\alpha \\
0, \text { if } \beta \neq \alpha
\end{array}\right. \\
& r_{\alpha}(P+Q)=_{\operatorname{def}} r_{\alpha}(P)+r_{\alpha}(Q) \\
& r_{\alpha}\left(P \bowtie_{L} Q\right)=_{\text {def }}\left\{\begin{array}{l}
\min \left(r_{\alpha}(P), r_{\alpha}(Q)\right) \text { if } \alpha \in L \\
r_{\alpha}(P)+r_{\alpha}(Q), \text { if } \alpha \notin L
\end{array}\right. \\
& r\left(\alpha, \lambda_{1}, \lambda_{2}, P, Q\right)==_{\text {def }} \frac{\lambda_{1}}{r_{\alpha}(P)} \cdot \frac{\lambda_{2}}{r_{\alpha}(Q)} \cdot \min \left(r_{\alpha}(P), r_{\alpha}(Q)\right)
\end{aligned}
$$




$$
\begin{aligned}
& \underset{\langle a, \lambda\rangle . P \stackrel{\alpha, \lambda}{\longrightarrow} P}{P} \frac{P \stackrel{\alpha, \lambda}{\longrightarrow} R}{P+Q \stackrel{\alpha, \lambda}{\longrightarrow} R} \quad \frac{Q \stackrel{\alpha, \lambda}{\longrightarrow} R}{P+Q \stackrel{\alpha, \lambda}{\longrightarrow} R} \quad \frac{P \stackrel{\alpha, \lambda}{\longrightarrow} Q, X:=P}{X \stackrel{\alpha, \lambda}{\longrightarrow} Q} \\
& \frac{P \stackrel{\alpha, \lambda}{\longrightarrow} P^{\prime}, \alpha \notin L}{P\left\|_{L} Q \stackrel{\alpha, \lambda}{\longrightarrow} P^{\prime}\right\|_{L} Q} \quad \frac{Q \stackrel{\alpha, \lambda}{\longrightarrow} Q^{\prime}, \alpha \notin L}{P\left\|_{L} Q \stackrel{\alpha, \lambda}{\longrightarrow} P\right\|_{L} Q^{\prime}} \\
& \frac{P \stackrel{\alpha, \lambda_{1}}{\longrightarrow} P^{\prime}, Q \stackrel{\alpha, \lambda_{2}}{\longrightarrow} Q^{\prime}, \alpha \in L}{P\left\|_{L} Q \stackrel{\alpha, r\left(\alpha, \lambda_{1}, \lambda_{2}, P, Q\right)}{\longrightarrow} P^{\prime}\right\|_{L} Q^{\prime}}
\end{aligned}
$$

Fig. 19. SOS Rules for $\mathrm{PEPA}_{k}$.

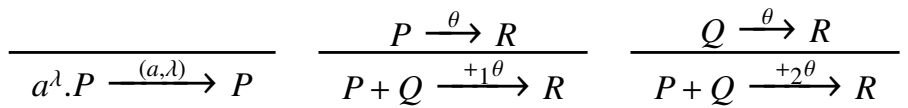

$$
\begin{aligned}
& \frac{P \stackrel{\theta}{\longrightarrow} P^{\prime}}{P\left|Q \stackrel{\|_{1} \theta}{\longrightarrow} P^{\prime}\right| Q} \quad \frac{Q \stackrel{\theta}{\longrightarrow} Q^{\prime}}{P\left|Q \stackrel{\|_{2} \theta}{\longrightarrow} P\right| Q^{\prime}} \\
& \frac{P \stackrel{\theta_{1}\left(a, \lambda_{1}\right)}{\longrightarrow} P^{\prime}, Q \stackrel{\theta_{2}\left(\bar{a}, \lambda_{2}\right)}{\longrightarrow} Q^{\prime}}{P\left|Q \stackrel{\left\langle{ }_{1} \theta_{1}\left(a, \lambda_{1}\right),\left.\right|_{2} \theta_{2}\left(\bar{a}, \lambda_{2}\right)\right\rangle r\left(x, \lambda_{1}, \lambda_{2}, P, Q\right)}{\longrightarrow} P^{\prime}\right| Q^{\prime}}
\end{aligned}
$$

Fig. 20. Rules for StoCCS.

In the original definition of PEPA [Hillston 1996], the rated-action prefix and parallel composition operators are denoted by $(a, \lambda) . P$ and $\bowtie_{L}$ respectively.

\section{A.5 Stoccs}

In the proved operational semantics, StoCCS is defined by the rules of Fig. 20 where $\theta$ ranges over derivation proofs, e.g. represented by terms of the following grammar:

\begin{tabular}{l|l|l|l|l|l}
$\theta:=(a, \lambda)$ & $+_{1} \theta$ & $+_{2} \theta$ & $\|_{1} \theta$ & $\|_{2} \theta$ & $\left\langle\left\|_{1} \theta,\right\|_{2} \theta\right\rangle$
\end{tabular}

Function $r\left(\alpha, \lambda_{1}, \lambda_{2}, P, Q\right)$, used to compute the rate of a synchronization, is defined as for PEPA when the minimum synchronization rate approach is used, while it is simply defined as $\lambda_{1} \cdot \lambda_{2}$ when the multiplicative rate approach is used.

From the LTS induced by the SOS rules an action labelled CTMC can be derived, by removing the proofs from the labels while summing up the rates of identical transitions.

In the original definition of Stochastic CCS reported in Section 2.2 of [Klin and Sassone 2008], the rated-output-action prefix, rated-input-action prefix and parallel composition operators are denoted by $(\bar{a}, \lambda) . P,(a, \lambda) . P$ and $\|$ respectively.

ACM Computing Surveys - In Press, Vol. , No., November 2012. 


$$
\begin{aligned}
& \frac{\stackrel{P}{\rightarrow} R}{a . P \stackrel{a}{\rightarrow} P} \quad \frac{Q \stackrel{a}{\rightarrow} R}{P+Q \stackrel{a}{\rightarrow} R} \quad \frac{P \stackrel{a}{\rightarrow} Q, X:=P}{X+Q \stackrel{a}{\rightarrow} R} \quad \frac{\stackrel{a}{\rightarrow} Q}{P} \\
& \frac{P \stackrel{a}{\rightarrow} P^{\prime}, a \notin L}{P\left\|_{L} Q \stackrel{a}{\rightarrow} P^{\prime}\right\|_{L} Q} \quad \frac{Q \stackrel{a}{\rightarrow} Q^{\prime}, a \notin L}{P\left\|_{L} Q \stackrel{a}{\rightarrow} P\right\|_{L} Q^{\prime}} \quad \frac{P \stackrel{a}{\rightarrow} P^{\prime}, Q \stackrel{a}{\rightarrow} Q^{\prime}, a \in L}{P\left\|_{L} Q \stackrel{a}{\rightarrow} P^{\prime}\right\|_{L} Q^{\prime}}
\end{aligned}
$$

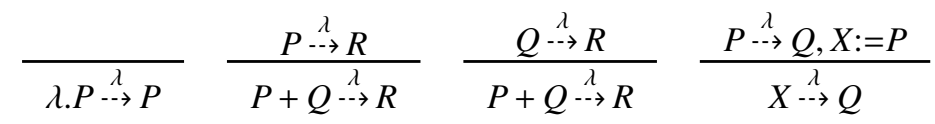

$$
\begin{aligned}
& \frac{\stackrel{\lambda}{P \rightarrow} P^{\prime}}{P\left\|_{L} Q_{\stackrel{\lambda}{\rightarrow}}^{\rightarrow} P^{\prime}\right\|_{L} Q} \quad \frac{\stackrel{\lambda}{Q \stackrel{\lambda}{\rightarrow} Q^{\prime}}}{P\left\|_{L} Q_{\stackrel{\lambda}{\rightarrow}}^{\rightarrow} P\right\|_{L} Q^{\prime}}
\end{aligned}
$$

Fig. 21. SOS Rules for the $\mathrm{IML}_{k}$.

A.6 $\quad \mathrm{IML}_{k}$

The SOS definition of $\mathrm{IML}_{k}$ (see Sect.8.1) is given in Fig. 21. The action transition relation $\rightarrow \subset \mathcal{P}_{I M L_{k}} \times \mathcal{A} \times \mathcal{P}_{I M L_{k}}$ is the least relation and the Markovian transition relation $\rightarrow \subset$ $\mathcal{P}_{I M L_{k}} \times \mathbb{R}_{>0} \times \mathcal{P}_{I M L_{k}}$ is the least multi-relation given by the rules in Fig. 21 .

Notice that in [Hermanns 2002] parallel composition (and hiding) are not defined by means of an explicit set of SOS rules, but, being derived operators, it is defined by means of expansion laws (and specific laws for hiding). Here we preferred to use explicit SOS rules for uniformity reasons and because we do not address equivalence relations.

In the original definition of IML [Hermanns 2002], the parallel composition operator is denoted by $|[L]|$.

\section{B. PROOFS RELATED TO SECT. 4}

\section{B.1 Proof of Proposition 4.1}

Proposition 4.1. For all $P \in \mathcal{P}_{C T M C}$ and $\mathscr{P} \in \mathbf{T F}\left(\mathcal{P}_{C T M C}, \mathbb{R}_{\geq 0}\right)$, if $P \stackrel{\delta^{e}}{\mapsto} \mathscr{P}$ can be derived from the rules of Fig. 3, then $\mathscr{P} \in \mathbf{F T F}\left(\mathscr{P}_{C T M C}, \mathbb{R}_{\geq 0}\right)$.

Proof. By derivation induction. Let $n \geq 1$ be the length of the derivation for proving $P \stackrel{\delta^{e}}{\rightarrow} \mathscr{P}$.

Base case: Trivial since the only cases in which $P \stackrel{\delta^{e}}{\longmapsto} \mathscr{P}$ can be derived with a proof of length 1 are those in which $\mathscr{P}=[]_{\mathbb{R}_{20}}$ or $\mathscr{P}=\left[P^{\prime} \mapsto \lambda\right]$ and []$,\left[P^{\prime} \mapsto \lambda\right] \in$ $\mathbf{F T F}\left(\mathcal{P}_{C T M C}, \mathbb{R}_{\geq 0}\right)$ by definition.

Inductive step: The last assert of any proof of length $n>1$ must be of the form $P+Q \stackrel{\delta^{e}}{\mapsto}$ $\mathscr{P}+\mathscr{Q}$ or $X \stackrel{\delta^{e}}{\rightarrow} \mathscr{P}$. In the first case $\mathscr{P}+\mathscr{Q} \in \mathbf{F T F}\left(\mathcal{P}_{C T M C}, \mathbb{R}_{\geq 0}\right)$, since $\mathscr{P}, \mathscr{Q} \in$ $\operatorname{FTF}\left(\mathcal{P}_{C T M C}, \mathbb{R}_{\geq 0}\right)$ by I.H. and $\mathbf{F T F}\left(\mathcal{P}_{C T M C}, \mathbb{R}_{\geq 0}\right)$ is closed under + by definition of + . In the second case the assert trivially follows from the I.H. 


\section{B.2 Proof of Theorem 4.3}

Theorem 4.3. $\mathcal{R}_{C T M C}$ is total and deterministic.

Proof. $\mathcal{R}_{\text {CTMC }}$ is total: By structural induction ${ }^{9}$, taking inaction and rate prefix as base cases, for which the assert is trivially proven. For the inductive step we show only the case $P+Q$ which is also very simple because $P \stackrel{\delta^{e}}{\rightarrow} \mathscr{P}$ and $Q \stackrel{\delta^{e}}{\rightarrow} \mathscr{Q}$, for some $\mathscr{P}$ and $\mathscr{Q}$ by the I.H., hence $P+Q \stackrel{\delta^{e}}{\rightarrow} \mathscr{P}+\mathscr{Q}$ by the FuTS semantics of the CTMC Language (Fig. 3).

$\mathcal{R}_{C T M C}$ is deterministic: By structural induction. We prove only the inductive step for case $P+Q$ here, the others being simpler.

$$
\begin{aligned}
& P+Q \stackrel{\delta^{e}}{\mapsto} \mathscr{R}_{1}, P+Q \stackrel{\delta^{e}}{\mapsto} \mathscr{R}_{2} \\
& \Rightarrow \quad\{\text { Def. of } \mapsto(\text { Fig. 3) }\} \\
& \mathscr{R}_{1}=\mathscr{P}_{1}+\mathscr{Q}_{1}, \mathscr{R}_{2}=\mathscr{P}_{2}+\mathscr{Q}_{2}, P \stackrel{\delta^{e}}{\rightarrow} \mathscr{P}_{1}, Q \stackrel{\delta^{e}}{\mapsto} \mathscr{Q}_{1}, P \stackrel{\delta^{e}}{\mapsto} \mathscr{P}_{2}, Q \stackrel{\delta^{e}}{\mapsto} \mathscr{Q}_{2} \\
& \text { for some } \mathscr{P}_{1}, \mathscr{Q}_{1}, \mathscr{P}_{2} \text { and } \mathscr{Q}_{2} \\
& \Rightarrow \quad\{\text { I.H. }\} \\
& \mathscr{P}_{1}=\mathscr{P}_{2}, \mathscr{Q}_{1}=\mathscr{Q}_{2}, \mathscr{R}_{1}=\mathscr{P}_{1}+\mathscr{Q}_{1}, \mathscr{R}_{2}=\mathscr{P}_{2}+\mathscr{Q}_{2} \\
& \Rightarrow \quad\{\text { Algebra }\} \\
& \mathscr{R}_{1}=\mathscr{R}_{2}
\end{aligned}
$$

\section{PROOFS RELATED TO SECT. 6}

\section{C.1 Proof of Proposition 6.1}

Proposition 6.1. For all $P \in \mathcal{P}_{T_{I I P P_{k}}}, \alpha \in \mathcal{L}_{T I P P_{k}}$ and $\mathscr{P} \in \mathbf{T F}\left(\mathcal{P}_{T I P P_{k}}, \mathbb{R}_{\geq 0}\right)$, if $P \stackrel{\alpha}{\longmapsto} \mathscr{P}$ can be derived using the set of rules composed only of rules (NIL), (CHO) and (CNS) of Figure 3 plus those of Figure 6 , then $\mathscr{P} \in \mathbf{F T F}\left(\mathscr{P}_{T I P P_{k}}, \mathbb{R}_{\geq 0}\right)$.

Proof. By induction on the length of the derivation for $P \stackrel{\alpha}{\rightarrow} \mathscr{P}$. We prove only the inductive step. The last assert of any derivation of length $n>1$ must be of the form $P+Q \stackrel{\alpha}{\rightarrow} \mathscr{P}+\mathscr{Q}$, or $P \|_{L} Q \stackrel{\alpha}{\rightarrow}\left(\mathscr{P} \otimes_{\|_{L}}(X Q)\right)+\left((X P) \otimes_{\|_{L}} \mathscr{Q}\right)$, or $P \|_{L} Q \stackrel{\alpha}{\longmapsto}$ $\mathscr{P} \|_{L} \mathscr{Q}$, or $X \stackrel{\alpha}{\rightarrow} \mathscr{P}$. In all cases the assert follows from Proposition 5.1 since $\mathscr{P}, \mathscr{Q} \in$ $\mathbf{F T F}\left(\mathcal{P}_{T I P P_{k}}, \mathbb{R}_{\geq 0}\right)$ by I.H. and $\left(\mathcal{X}_{\mathbb{R}_{\geq 0}} P\right),\left(\mathcal{X}_{\mathbb{R}_{\geq 0}} Q\right) \in \mathbf{F T F}\left(\mathcal{P}_{T I P P_{k}}, \mathbb{R}_{\geq 0}\right)$ by definition.

\section{C.2 Proof of Theorem 6.3}

Theorem 6.3. $\mathcal{R}_{\text {TIPP }_{k}}$ is total and deterministic.

Proof. $\mathcal{R}_{\text {TIPP }_{k}}$ is total: By induction on the structure, taking inaction and rated action prefix as base cases, for which the assert is trivially proven. For the inductive step

\footnotetext{
${ }^{9}$ Strictly speaking, the proof is carried on by guarded induction, thanks to the guarded recursion constraint on the syntax. This amounts to assuming a function $\phi: \mathcal{P}_{C T M C} \rightarrow \mathbb{N}$ such that $\phi\left(P_{1}\right.$ op $\left.P 2\right)>\operatorname{Max}\left\{\left(\phi P_{1}\right),\left(\phi P_{2}\right)\right\}$ for all syntactic operators $o p$ and, moreover $\phi X>\phi P$ if $X:=P$. In the sequel we will keep the structural induction terminology.
}

ACM Computing Surveys - In Press, Vol. , No. , November 2012. 
we show only the case $P \quad \|_{L} \quad Q$ which is also very simple because $P \stackrel{\alpha}{\mapsto} \mathscr{P}$ and $Q \stackrel{\alpha}{\longmapsto} \mathscr{Q}$, for some $\mathscr{P}$ and $\mathscr{Q}$ by the I.H., hence, assuming $(n \alpha) \notin L, P \|_{L} \quad Q \stackrel{\alpha}{\mapsto}$ $\left(\mathscr{P} \otimes_{\|_{L}}(\mathcal{X} Q)\right)+\left((\mathcal{X} P) \otimes_{\|_{L}} \mathscr{Q}\right)$ by the FuTS semantics of TIPP ${ }_{k}$; the case for $(n \alpha) \in L$ is similar.

$\mathcal{R}_{T I P P_{k}}$ is deterministic: By structural induction. We prove only the inductive step for case $P \|_{L} Q$ here, the others being similar or simpler. Let us suppose there are two dif-

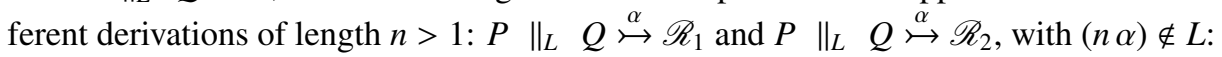

$$
\begin{aligned}
& P\left\|_{L} Q \stackrel{\alpha}{\mapsto} \mathscr{R}_{1}, P\right\|_{L} Q \stackrel{\alpha}{\mapsto} \mathscr{R}_{2} \\
& \Rightarrow \quad \text { Def. of } \nrightarrow\} \\
& \mathscr{R}_{1}=\left(\mathscr{P}_{1} \otimes_{\|_{L}}(X Q)\right)+\left((X P) \otimes_{\|_{L}} \mathscr{Q}_{1}\right), \\
& \mathscr{R}_{2}=\left(\mathscr{P}_{2} \otimes_{\|_{L}}(\mathcal{X} Q)\right)+\left((X P) \otimes_{\|_{L}} \mathscr{Q}_{2}\right) \\
& \text { for some } \mathscr{P}_{1}, \mathscr{Q}_{1}, \mathscr{P}_{2}, \mathscr{Q}_{2} \\
& \Rightarrow \quad\{\text { I.H. }\} \\
& \mathscr{P}_{1}=\mathscr{P}_{2}, \mathscr{Q}_{1}=\mathscr{Q}_{2}, \\
& \mathscr{R}_{1}=\left(\mathscr{P}_{1} \otimes_{\|_{L}}\left(X_{\mathbb{R}_{\geq 0}} Q\right)\right)+\left(\left(X_{\mathbb{R}_{\geq 0}} P\right) \otimes_{\|_{L}} \mathscr{Q}_{1}\right), \\
& \mathscr{R}_{2}=\left(\mathscr{P}_{2} \otimes_{\|_{L}}\left(\mathcal{X}_{\mathbb{R}_{\geq 0}} Q\right)\right)+\left(\left(\mathcal{X}_{\mathbb{R}_{\geq 0}} P\right) \otimes_{\|_{L}} \mathscr{Q}_{2}\right) \\
& \Rightarrow \quad\{\text { Algebra }\} \\
& \mathscr{R}_{1}=\mathscr{R}_{2}
\end{aligned}
$$

The case $P \|_{L} Q \stackrel{\alpha}{\mapsto} \mathscr{R}_{1}$ and $P \|_{L} Q \stackrel{\alpha}{\mapsto} \mathscr{R}_{2}$, with $(n \alpha) \in L$ is similar.

\section{C.3 Proof of Theorem 6.4}

Theorem 6.4. For all $P, Q \in \mathcal{P}_{T I P P_{k}}, \alpha \in \mathcal{L}_{T I P P_{k}}$, and unique $\mathscr{P} \in \mathbf{F T F}\left(\mathcal{P}_{T I P P_{k}}, \mathbb{R}_{\geq 0}\right)$ such that $P \stackrel{\alpha}{\mapsto} \mathscr{P}$ the following holds: $(\mathscr{P} Q)=\mathbf{r t}_{(n \alpha)}(P, Q)$

Proof. By induction of the length of the derivation for $P \stackrel{\alpha}{\hookrightarrow} \mathscr{P}$. We prove only the inductive step for case $P_{1} \|_{L} P_{2}$, under the assumption $(n \alpha) \in L$, the other cases being similar. By definition of the FuTS semantics of TIPP $k$, the last assert of the derivation is of the form $P_{1} \|_{L} P_{2} \stackrel{\alpha}{\longmapsto} \mathscr{P}_{1} \otimes_{\|_{L}} \mathscr{P}_{2}$, with $P_{1} \stackrel{\alpha}{\longmapsto} \mathscr{P}_{1}$ and $P_{2} \stackrel{\alpha}{\longmapsto} \mathscr{P}_{2}$. We observe that if $Q$ is not of the form $Q_{1} \|_{L} Q_{2}$ then $\left(\mathscr{P}_{1} \otimes_{\|_{L}} \mathscr{P}_{2}\right) Q=0$. On the other hand, we observe that the only transitions from $P_{1} \|_{L} P_{2}$ allowed by the SOS semantics of TIPP ${ }_{k}$ are to terms of the form $Q_{1} \|_{L} Q_{2}$, so also $\mathbf{r t}_{(n \alpha)}\left(P_{1} \|_{L} P_{2}, Q\right)=0$ if $Q$ is not of the form $Q_{1} \|_{L} Q_{2}$. Let us assume $Q$ is of the form $Q_{1} \|_{L} Q_{2}$.

$$
\begin{aligned}
& \left(\mathscr{P}_{1} \otimes_{\|_{L}} \mathscr{P}_{2}\right) Q_{1} \|_{L} Q_{2} \\
= & \left\{\text { Def. }\left(\mathscr{P}_{1} \otimes_{\|_{L}} \mathscr{P}_{2}\right)\right\}
\end{aligned}
$$




$$
\begin{aligned}
& \left(\mathscr{P}_{1} Q_{1}\right) \cdot\left(\mathscr{P}_{2} Q_{2}\right) \\
= & \left\{P_{1} \stackrel{\alpha}{\mapsto} \mathscr{P}_{1} \text { and } P_{2} \stackrel{\alpha}{\mapsto} \mathscr{P}_{2} ; \text { I.H. }\right\} \\
& \operatorname{rt}_{(n \alpha)}\left(P_{1}, Q_{1}\right) \cdot \mathbf{r t}_{(n \alpha)}\left(P_{2}, Q_{2}\right)
\end{aligned}
$$

$=\left\{\right.$ SOS definition of $\operatorname{TIPP}_{k}$; Def. of $\left.\mathbf{r t}_{a}\right\}$

$$
\mathbf{r t}_{(n \alpha)}\left(P_{1}\left\|_{L} P_{2}, Q_{1}\right\|_{L} Q_{2}\right)
$$

\section{C.4 Proof of Proposition 6.5}

Proposition 6.5. For all $P \in \mathcal{P}_{E M P A_{k}}, \alpha \in \mathcal{L}_{E M P A_{k}}$, and $\mathscr{P} \in \mathbf{T F}\left(\mathcal{P}_{E M P A_{k}}, \mathbb{R}_{\geq 0}\right)$, if $P \stackrel{\alpha}{\longmapsto} \mathscr{P}$ can be derived using the set of rules composed only of rules (NIL), (CHO) and (CNS) of Figure 3 plus rules (RAPF1) and (RAPF2) and (PAR1) of Figure 6 and those of Figure 7, then $\mathscr{P} \in \mathbf{F T F}\left(\mathcal{P}_{E M P A_{k}}, \mathbb{R}_{\geq 0}\right)$

Proof. By derivation induction. Let $n \geq 1$ be the length of the derivation for proving $P \stackrel{\alpha}{\hookrightarrow} \mathscr{P}$.

Base case: Trivial since the only cases in which $P \stackrel{\alpha}{\rightarrow} \mathscr{P}$ can be derived with a proof of length 1 are those in which $\mathscr{P}=[]_{\mathbb{R}_{\geq 0}}$ or $\mathscr{P}=\left[P^{\prime} \mapsto x\right]$, with $x \in \mathbb{R}_{>0}$, and []$_{\mathbb{R}_{\geq 0}} \in$ $\mathbf{F T F}\left(\mathcal{P}_{E M P A_{k}}, \mathbb{R}_{\geq 0}\right)$ and $\left[P^{\prime} \mapsto x\right] \in \mathbf{F T F}\left(\mathcal{P}_{E M P A_{k}}, \mathbb{R}_{\geq 0}\right)$ by definition.

Inductive step: The last assert of any proof of length $n>1$ must be of the form $P+Q \stackrel{\alpha}{\mapsto}$ $\mathscr{P}+\mathscr{Q}$, or $P \|_{L} Q \stackrel{\alpha}{\longmapsto}\left(\mathscr{P} \otimes_{\|_{L}}(X Q)\right)+\left((X P) \otimes_{\|_{L}} \mathscr{Q}\right)$, or $P \|_{L} Q \stackrel{\delta_{a *}^{e}}{\longmapsto} \mathscr{P} \otimes_{\|_{L}} \mathscr{Q}$. $\frac{(\oplus \mathscr{P})+(\oplus \mathscr{Q})}{(\oplus \mathscr{P}) \cdot(\oplus \mathscr{Q})}$, or $P \|_{L} \quad Q \stackrel{\delta_{a}^{e}}{\rightarrow} \mathscr{P}_{o} \otimes_{\|_{L}} \mathscr{Q}_{i} \cdot \frac{1}{\oplus \mathscr{Q}_{i}}+\mathscr{P}_{i} \otimes_{\|_{L}} \mathscr{Q}_{o} \cdot \frac{1}{\oplus \mathscr{P}_{i}}$, or, finally, $X \stackrel{\alpha}{\longmapsto}$ $\mathscr{P}$. In all cases the assert follows using Proposition 5.1 since $\mathscr{P}, \mathscr{Q}, \mathscr{P}_{o}, \mathscr{P}_{i}, \mathscr{Q}_{o}, \mathscr{Q}_{i} \in$ $\mathbf{F T F}\left(\mathcal{P}_{E M P A_{k}}, \mathbb{R}_{\geq 0}\right)$ by I.H. and $\left(\mathcal{X}_{\mathbb{R}_{\geq 0}} P\right),\left(\mathcal{X}_{\mathbb{R}_{\geq 0}} Q\right) \in \mathbf{F T F}\left(\mathcal{P}_{E M P A_{k}}, \mathbb{R}_{\geq 0}\right)$ by definition.

\section{C.5 Proof of Theorem 6.7}

Theorem 6.7. $\mathcal{R}_{E M P A_{k}}$ is total and deterministic.

Proof. By structural induction. The proof is similar to that of Theorem 6.3.

\section{C.6 Proof of Theorem 6.8}

Theorem 6.8. For all $P, Q \in \mathcal{P}_{E M P A_{k}}, \delta_{a}^{e}, \delta_{a *}^{e} \in \mathcal{L}_{E M P A_{k}}$, and unique functions $\mathscr{P}, \mathscr{P}^{\prime} \in$ $\operatorname{FTF}\left(\mathcal{P}_{E M P A_{k}}, \mathbb{R}_{\geq 0}\right)$ such that $P \stackrel{\delta_{a}^{e}}{\hookrightarrow} \mathscr{P}$ and $P \stackrel{\delta_{a *}^{e}}{\rightarrow} \mathscr{P}^{\prime}$, the following holds: $(\mathscr{P} Q)=$ $\mathbf{r t}_{a}(P, Q),\left(\mathscr{P}^{\prime} Q\right)=\mathbf{w t}_{a}(P, Q)$, and $\left(\oplus \mathscr{P}^{\prime}\right)=\operatorname{weight}(P, a)$.

Proof. We prove the assert by induction on the length of the derivations for $P \stackrel{\delta_{a}^{e}}{\hookrightarrow} \mathscr{P}$ and for $P \stackrel{\delta_{a *}^{e}}{\rightarrow} \mathscr{P}$. We prove only the inductive step for case $P_{1} \|_{L} P_{2}$, under the assumption $a \in L$, the other cases being similar. By definition of the FuTS semantics of EMPA ${ }_{k}$, the last asserts of the derivations are of the form

$P_{1} \|_{L} P_{2} \stackrel{\delta_{a}^{e}}{\rightarrow} \mathscr{P}_{1}^{0} \otimes_{\|_{L}} \mathscr{P}_{2}^{i} \cdot \frac{1}{\oplus \mathscr{P}_{2}^{i}}+\mathscr{P}_{1}^{i} \otimes_{\|_{L}} \mathscr{P}_{2}^{o} \cdot \frac{1}{\oplus \mathscr{P}_{1}^{i}}$ and

$P_{1} \|_{L} P_{2} \stackrel{\delta_{a *}^{e}}{\longmapsto} \mathscr{P}_{1} \otimes_{\|_{L}} \mathscr{P}_{2} \cdot \frac{\left(\oplus \mathscr{P}_{1}\right)+\left(\oplus \mathscr{P}_{2}\right)}{\left(\oplus \mathscr{P}_{1}\right) \cdot\left(\oplus \mathscr{P}_{2}\right)}$

ACM Computing Surveys - In Press, Vol. , No. , November 2012. 
We observe that if $Q$ is not of the form $Q_{1} \|_{L} Q_{2}$ then $\left(\mathscr{P}_{1}^{o} \otimes_{\|_{L}} \mathscr{P}_{2}^{i}\right) Q=0$, as well as $\left(\mathscr{P}_{1}^{i} \otimes_{\|_{L}} \mathscr{P}_{2}^{o}\right) Q$, and $\left(\mathscr{P}_{1} \otimes_{\|_{L}} \mathscr{P}_{2}\right) Q$. On the other hand, we observe that the only transitions from $P_{1} \|_{L} P_{2}$ allowed by the original SOS semantics of EMPA $k$ are to terms of the form $Q_{1} \|_{L} Q_{2}$, so also $\mathbf{r t}_{a}\left(P_{1} \|_{L} P_{2}, Q\right)=\mathbf{w t}_{a}\left(P_{1} \|_{L} P_{2}, Q\right)=0$ if $Q$ is not of the form $Q_{1} \|_{L} Q_{2}$. Let us assume $Q$ is of the form $Q_{1} \|_{L} Q_{2}$.

$$
\begin{aligned}
& \left(\begin{array}{l}
\left(\mathscr{P}_{1}^{o} \otimes_{\|_{L}} \mathscr{P}_{2}^{i} \cdot \frac{1}{\oplus \mathscr{P}_{2}^{i}}+\mathscr{P}_{1}^{i} \otimes_{\|_{L}} \mathscr{P}_{2}^{o} \cdot \frac{1}{\oplus \mathscr{P}_{1}^{i}}\right) Q_{1} \|_{L} Q_{2} \\
\left(\mathscr{P}_{1} \otimes_{\|_{L}} \mathscr{P}_{2} \cdot \frac{\left(\oplus \mathscr{P}_{1}\right)+\left(\oplus \mathscr{P}_{2}\right)}{\left(\oplus \mathscr{P}_{1}\right) \cdot\left(\oplus \mathscr{P}_{2}\right)}\right) Q_{1} \|_{L} Q_{2} \\
\oplus\left(\left(\mathscr{P}_{1} \otimes_{\|_{L}} \mathscr{P}_{2}\right) \cdot \frac{\left(\oplus \mathscr{P}_{1}\right)+\left(\oplus \mathscr{P}_{2}\right)}{\left(\oplus \mathscr{P}_{1}\right) \cdot\left(\oplus \mathscr{P}_{2}\right)}\right)
\end{array}\right) \\
& =\quad\left\{\text { Def. } \otimes_{\|_{L}}, \oplus\right\} \\
& \left(\begin{array}{l}
\left(\mathscr{P}_{1}^{o} Q_{1}\right) \cdot\left(\mathscr{P}_{2}^{i} Q_{2}\right) \cdot \frac{1}{\oplus \mathscr{P}_{2}^{i}}+\left(\mathscr{P}_{1}^{i} Q_{1}\right) \cdot\left(\mathscr{P}_{2}^{o} Q_{2}\right) \cdot \frac{1}{\oplus \mathscr{P}_{1}^{i}} \\
\left(\mathscr{P}_{1} Q_{1}\right) \cdot\left(\mathscr{P}_{2} Q_{2}\right) \cdot \frac{\left(\oplus \mathscr{P}_{1}\right)+\left(\oplus \mathscr{P}_{2}\right)}{\left(\oplus \mathscr{P}_{1}\right) \cdot\left(\oplus \mathscr{P}_{2}\right)} \\
\frac{\left(\oplus \mathscr{P}_{1}\right)+\left(\oplus \mathscr{P}_{2}\right)}{\left(\oplus \mathscr{P}_{1}\right) \cdot\left(\oplus \mathscr{P}_{2}\right)} \cdot \sum_{Q_{1}^{\prime} \|_{L} Q_{2}^{\prime} \in \mathcal{P}_{\text {MPPA }_{k}}}\left(\mathscr{P}_{1} Q_{1}^{\prime}\right) \cdot\left(\mathscr{P}_{2} Q_{2}^{\prime}\right)
\end{array}\right) \\
& =\quad\left\{P_{h} \stackrel{\delta_{a *}^{e}}{\longmapsto} \mathscr{P}_{h}^{i}, P_{h} \stackrel{\delta_{a *}^{e}}{\longmapsto} \mathscr{P}_{h}, h=1,2 \text {; Unicity corollary (Th. 6.7) }\right\} \\
& \left(\begin{array}{l}
\left(\mathscr{P}_{1}^{o} Q_{1}\right) \cdot\left(\mathscr{P}_{2}^{i} Q_{2}\right) \cdot \frac{1}{\oplus \mathscr{P}_{2}^{i}}+\left(\mathscr{P}_{1}^{i} Q_{1}\right) \cdot\left(\mathscr{P}_{2}^{o} Q_{2}\right) \cdot \frac{1}{\oplus \mathscr{P}_{1}^{i}} \\
\left(\mathscr{P}_{1}^{i} Q_{1}\right) \cdot\left(\mathscr{P}_{2}^{i} Q_{2}\right) \cdot \frac{\left(\oplus \mathscr{P}_{1}^{i}\right)+\left(\oplus \mathscr{P}_{2}^{i}\right)}{\left(\oplus \mathscr{P}_{1}^{i}\right) \cdot\left(\oplus \mathscr{P}_{2}^{i}\right)} \\
\frac{\left(\oplus \mathscr{P}_{1}^{i}\right)+\left(\oplus \mathscr{P}_{2}^{i}\right)}{\left(\oplus \mathscr{P}_{1}^{i}\right) \cdot\left(\oplus \mathscr{P}_{2}^{i}\right)} \cdot \sum_{Q_{1}^{\prime} \|_{L} Q_{2}^{\prime} \in \mathcal{P}_{E M P A_{k}}}\left(\mathscr{P}_{1}^{i} Q_{1}^{\prime}\right) \cdot\left(\mathscr{P}_{2}^{i} Q_{2}^{\prime}\right)
\end{array}\right) \\
& =\quad\left\{P_{h} \stackrel{\delta_{a}^{e}}{\rightarrow} \mathscr{P}_{h}^{o}, P_{h} \stackrel{\delta_{a *}^{e}}{\rightarrow} \mathscr{P}_{h}^{i}, h=1,2 ; \text { I.H. }\right\} \\
& \left(\begin{array}{l}
\frac{\mathbf{r t}_{a}\left(P_{1}, Q_{1}\right) \cdot \mathbf{w t}_{a}\left(P_{2}, Q_{2}\right)}{w e i g h t\left(a, P_{2}\right)}+\frac{\mathbf{w t}_{a}\left(P_{1}, Q_{1}\right) \cdot \mathbf{r t}_{a}\left(P_{2}, Q_{2}\right)}{\text { weight }\left(a, P_{1}\right)} \\
\mathbf{w t}_{a}\left(P_{1}, Q_{1}\right) \cdot \mathbf{w t}_{a}\left(P_{2}, Q_{2}\right) \cdot \frac{\text { weight }\left(a, P_{1}\right)+\text { weight }\left(a, P_{2}\right)}{\operatorname{weight}\left(a, P_{1}\right) \cdot \operatorname{weight}\left(a, P_{2}\right)} \\
\frac{\text { weight }\left(a, P_{1}\right)+\text { weight }\left(a, P_{2}\right)}{\text { weight }\left(a, P_{1}\right) \cdot \operatorname{weight}\left(a, P_{2}\right)} \cdot \sum_{Q_{1}^{\prime} \|_{L} Q_{2}^{\prime} \in \mathcal{P}_{E M A_{k}}} \mathbf{w t}_{a}\left(P_{1}, Q_{1}^{\prime}\right) \cdot \mathbf{w t}_{a}\left(P_{2} Q_{2}^{\prime}\right)
\end{array}\right) \\
& =\quad\left\{\text { Def. } \mathbf{r t}_{a} \text {; Def. } \mathbf{w t}_{a} \text {; Def. weight; SOS definition of EMPA }\right\}
\end{aligned}
$$

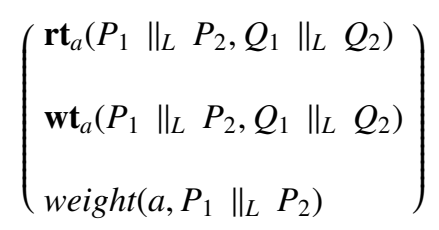




\section{C.7 Proof of Proposition 6.9}

Proposition 6.9. For all $P \in \mathcal{P}_{P_{E P A_{k}}}, \alpha \in \mathcal{L}_{P_{E P A_{k}}}$, and $\mathscr{P} \in \mathbf{T F}\left(\mathcal{P}_{P_{E P A_{k}}}, \mathbb{R}_{\geq 0}\right)$, if $P \stackrel{\alpha}{\longmapsto}$ $\mathscr{P}$ can be derived using the set of rules composed only of rules (CHO) and (CNS) of Figure 3, (RAPF1), (RAPF2) and (PAR1) of Figure 6 and rule (PAR2P) Figure 8, then $\mathscr{P} \in \mathbf{F T F}\left(\mathcal{P}_{\text {PEPA }_{k}}, \mathbb{R}_{\geq 0}\right)$.

Proof. By induction on the length of the derivation for $P \stackrel{\alpha}{\rightarrow} \mathscr{P}$. We prove only the inductive step. The last assert of any derivation of length $n>1$ must be of the form $P+Q \stackrel{\alpha}{\longmapsto} \mathscr{P}+\mathscr{Q}$, or $P \|_{L} Q \stackrel{\alpha}{\longmapsto}\left(\mathscr{P} \otimes_{\|_{L}}(X Q)\right)+\left((X P) \otimes_{\|_{L}} \mathscr{Q}\right)$, or $P \|_{L} Q \stackrel{\alpha}{\longmapsto}$ $\mathscr{P} \|_{L} \mathscr{Q}$, or $X \stackrel{\alpha}{\rightarrow} \mathscr{P}$. In all cases the assert follows from Proposition 5.1 since $\mathscr{P}, \mathscr{Q} \in$

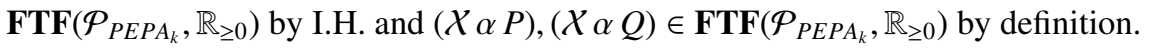

\section{C.8 Proof of Theorem 6.11}

Theorem 6.11. $\mathcal{R}_{{ }_{P_{E P A}}}$ is total and deterministic.

Proof. By structural induction. The proof is similar to that of Theorem 6.3.

\section{C.9 Proof of Theorem 6.12}

Theorem 6.12. For all $P, Q \in \mathcal{P}_{P E P A_{k}}, \alpha \in \mathcal{L}_{P E P A_{k}}$, and unique $\mathscr{P} \in \mathbf{F T F}\left(\mathcal{P}_{P E P A_{k}}, \mathbb{R}_{\geq 0}\right)$ such that $P \stackrel{\alpha}{\longmapsto} \mathscr{P}$ the following holds: $(\mathscr{P} Q)=\mathbf{r t}_{(n \alpha)}(P, Q)$

Proof. By induction of the length of the derivation for $P \stackrel{\alpha}{\longmapsto} \mathscr{P}$. The proof is similar to that of Theorem 6.4.

\section{PROOFS RELATED TO SECT. 7}

\section{D.1 Proof of Proposition 7.1}

Proposition 7.1. For all $P \in \mathcal{P}_{S_{t o C C S}}, \alpha \in \mathcal{L}_{S t o C C S_{A A}}$, and $\mathscr{P} \in \mathbf{T F}\left(\mathcal{P}_{S_{\text {toCCS }}}, \mathbb{R}_{\geq 0}\right)$ if $P \stackrel{\alpha}{\longmapsto} \mathscr{P}$ can be derived using the set of rules consisting of rules (NIL) and (CHO) of Figure 3, plus rules in Figure 9, and using one out of (PAR $\left.\mathrm{PA}_{\mathrm{AA}-\mathrm{M}}\right)$ and $\left(\mathrm{PAR}_{\mathrm{AA}-*}\right)$, then $\mathscr{P} \in \mathbf{F T F}\left(\mathscr{P}_{S_{\text {toCCS }}}, \mathbb{R}_{\geq 0}\right)$.

Proof. By induction on the length of the derivation for $P \stackrel{\alpha}{\longmapsto} \mathscr{P}$. We prove only the inductive step. The last assert of any derivation of length $n>1$ must be of the form

$$
\begin{aligned}
& -P+Q \stackrel{\alpha}{\hookrightarrow} \mathscr{P}+\mathscr{Q} \text {, or } \\
& -P \mid Q \stackrel{\alpha}{\mapsto}\left(\mathscr{P} \otimes_{\mid}(X Q)\right)+\left((X P) \otimes_{\mid} \mathscr{Q}\right) \text {, or } \\
& -P \mid Q \stackrel{\delta_{a}^{e}}{\rightarrow}\left(\mathscr{P} \otimes_{\mid}(X Q)\right)+\left((X P) \otimes_{\mid} \mathscr{Q}\right)+\mathscr{P}_{i} \otimes_{\mid} \mathscr{Q}_{o}+\mathscr{P}_{o} \otimes_{\mid} \mathscr{Q}_{i} \text {, or } \\
& -P \mid Q \stackrel{\delta_{a}^{e}}{\rightarrow}\left(\mathscr{P} \otimes_{\mid}(X Q)\right)+\left((X P) \otimes_{\mid} \mathscr{Q}\right)+\mathscr{P}_{i} \otimes_{\mid} \mathscr{Q}_{o}+\mathscr{P}_{o} \otimes_{\mid} \mathscr{Q}_{i} \text { (rule }\left(\mathrm{PAR}_{\mathrm{AA}-*}\right) \text { used), or }
\end{aligned}
$$

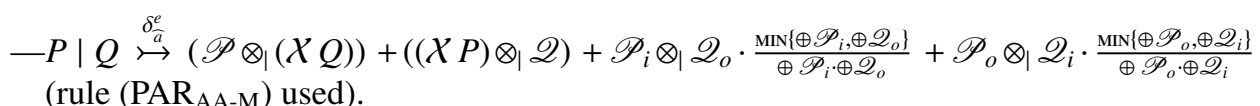

In all cases the assert follows using Proposition 5.1 since $\mathscr{P}, \mathscr{P}_{i}, \mathscr{P}_{0}, \mathscr{Q}, \mathscr{Q}_{i}, \mathscr{Q}_{o}$ are functions in $\mathbf{F T F}\left(\mathcal{P}_{S t o C C S}, \mathbb{R}_{\geq 0}\right)$ by I.H. and $(\mathcal{X P}),(\mathcal{X} Q) \in \mathbf{F T F}\left(\mathcal{P}_{S t o C C S_{I}}, \mathbb{R}_{\geq 0}\right)$ by definition. 


\section{D.2 Proof of Theorem 7.3}

Theorem 7.3. Both $\mathcal{R}_{S t o C C S_{A A}}^{*}$ and $\mathcal{R}_{S t o C C S_{A A}}^{M}$ are total and deterministic.

Proof. By structural induction. The proof is similar to that of Theorem 6.3.

\section{D.3 Proof of Theorem 7.4}

Theorem 7.4. Both in the minimum rate and in the multiplicative approach, for all $P, Q \in$ $\mathcal{P}_{\text {StoCCS }_{I}}, \alpha \in \mathcal{L}_{\text {StoCCS }_{I}}$, and unique $\mathscr{P} \in \mathbf{F T F}\left(\mathcal{P}_{\text {StoCCS }_{I}}, \mathbb{R}_{\geq 0}\right)$ such that $P \stackrel{\alpha}{\longmapsto} \mathscr{P}$ the following holds:

$$
(\mathscr{P} Q)=\left\{\begin{array}{l}
\mathbf{r t}_{a}(P, Q), \text { if } \alpha=\delta_{a}^{e} \text { with } a \in \mathcal{A} \cup \overline{\mathcal{A}} \\
\mathbf{r t}_{\langle a \mid \bar{a}\rangle}(P, Q)+\mathbf{r t}_{\langle\bar{a} \mid a\rangle}(P, Q), \text { if } \alpha=\delta_{\bar{a}}^{e} \text { with } a \in \mathcal{A}
\end{array}\right.
$$

Proof.

\section{Minimum rate approach:}

We prove only the inductive step for case $P_{1} \mid P_{2}$, under the assumption $\alpha=\delta_{\vec{a}}^{e}$ with $a \in$ $\mathcal{A}$, the other cases being similar. By definition of the FuTS semantics of StoCCS ${ }_{I}$, the last assert of the derivation is of the form $P_{1} \mid P_{2} \stackrel{\delta_{\tilde{a}}^{e}}{\rightrightarrows} \mathscr{R}$, where

$$
\begin{aligned}
& \mathscr{R}=\left(\mathscr{P}_{1} \otimes_{\mid}\left(X P_{2}\right)\right)+\left(\left(X P_{1}\right) \otimes_{\mid} \mathscr{P}_{2}\right)+\mathscr{P}_{1 i} \otimes_{\mid} \mathscr{P}_{2 o} \cdot \frac{\left.\operatorname{MiN} \mid \oplus \mathscr{P}_{1 i} \oplus \mathscr{P}_{2 o}\right\}}{\oplus \mathscr{P}_{1 i} \oplus \mathscr{P}_{2 o}}+\mathscr{P}_{1 o} \otimes_{\mid} \mathscr{P}_{2 i} \cdot \frac{\left.\operatorname{MiN} \mid \oplus \mathscr{P}_{10}, \oplus \mathscr{P}_{1 i}\right\}}{\oplus \mathscr{P}_{10} \cdot \oplus \mathscr{P}_{2 i}} \\
& \text { with } P_{1} \stackrel{\delta_{a}^{e}}{\rightarrow} \mathscr{P}_{1}, P_{1} \stackrel{\delta_{a}^{e}}{\rightarrow} \mathscr{P}_{1 i}, P_{1} \stackrel{\delta_{a}^{e}}{\rightarrow} \mathscr{P}_{1 o}, P_{2} \stackrel{\delta_{a}^{e}}{\rightarrow} \mathscr{P}_{2}, P_{2} \stackrel{\delta_{a}^{e}}{\rightarrow} \mathscr{P}_{2 i}, P_{2} \stackrel{\delta_{\bar{a}}^{e}}{\rightarrow} \mathscr{P}_{2 o} \text {. }
\end{aligned}
$$

We observe that if $Q$ is not of the form $Q_{1} \mid Q_{2}$ then $\mathscr{R} Q=0$. On the other hand, we observe that the only transitions from $P_{1} \mid P_{2}$ allowed by the SOS semantics of StoCCS are to terms of the form $Q_{1} \mid Q_{2}$, so also $\mathbf{r t}_{\alpha}\left(P_{1} \mid P_{2}, Q\right)=0$ if $Q$ is not of the form $Q_{1} \mid Q_{2}$. Let us assume $Q$ is of the form $Q_{1} \mid Q_{2}$. There are several cases to be considered depending to the fact that $\delta_{\vec{a}}^{e}$ denotes synchronisations

(1) between $P_{1}$ and $P_{2}$ where

(a) $P_{1}$ performs the input $a$ and $P_{2}$ performs the output $\bar{a}$, or

(b) $P_{1}$ performs the output $\bar{a}$ and $P_{2}$ performs the input $a$, or

(2) within

(a) $P_{1}$ alone, or

(b) $P_{2}$ alone

and are inherited by $P_{1} \mid P_{2}$.

We consider only the case of synchronisations between $P_{1}$ and $P_{2}$ where $P_{1}$ performs the input $a$ and $P_{2}$ performs the output $\bar{a}$, the other cases being similar or simpler. In this case, function $\mathscr{R}$ reduces to $\mathscr{P}_{1 i} \otimes_{\mid} \mathscr{P}_{2 o} \cdot \frac{\operatorname{Min}\left\{\oplus \mathscr{P}_{1 i} \oplus \mathscr{P}_{2 o}\right\}}{\oplus \mathscr{P}_{1 i} \oplus \mathscr{P}_{2 o}}$ since this is the sub-term of $\mathscr{R}$ dealing with such synchronisations between $P_{1}$ and $P_{2}$. We get the following derivation noting that, under our assumptions, $\oplus \mathscr{P}_{1 i} \cdot \oplus \mathscr{P}_{2 o} \neq 0$ :

$$
\left(\mathscr{P}_{1 i} \otimes_{\mid} \mathscr{P}_{2 o} \cdot \frac{\operatorname{MiN}\left\{\oplus \mathscr{P}_{1 i} \oplus \mathscr{P}_{2 o}\right\}}{\oplus \mathscr{P}_{1 i} \oplus \mathscr{P}_{2 o}}\right)\left(Q_{1} \mid Q_{2}\right)
$$




$$
\begin{aligned}
& =\quad\left\{\text { Def. } \mathscr{R}_{1} \otimes_{\mid} \mathscr{R}_{2} \cdot \frac{\operatorname{MiN}\left\{\oplus \mathscr{R}_{1}, \oplus \mathscr{R}_{2}\right\}}{\oplus \mathscr{R}_{1} \cdot \oplus \mathscr{R}_{2}} \text { in } \mathbf{F T F}\left(\mathcal{P}_{S t o C C S}, \mathbb{R}_{\geq 0}\right)\right\} \\
& \left(\mathscr{P}_{1 i} Q_{1}\right) \cdot\left(\mathscr{P}_{2 o} Q_{2}\right) \cdot \frac{\operatorname{MiN}\left\{\oplus \mathscr{P}_{1 i} \oplus \mathscr{P}_{2 o}\right\}}{\oplus \mathscr{P}_{1 i} \oplus \mathscr{P}_{2 o}} \\
& =\left\{P_{1} \stackrel{\delta_{a}^{e}}{\rightrightarrows} \mathscr{P}_{1 i}, P_{2} \stackrel{\delta_{\tilde{u}}^{e}}{\mapsto} \mathscr{P}_{2 o} ; \text { I.H. }\right\} \\
& \mathbf{r t}_{a}\left(P_{1}, Q_{1}\right) \cdot \mathbf{r t}_{\bar{a}}\left(P_{2}, Q_{2}\right) \cdot \frac{\operatorname{Min}\left\{\oplus \mathscr{P}_{1 i} \oplus \mathscr{P}_{2 o}\right\}}{\oplus \mathscr{P}_{1 i}: \oplus \mathscr{P}_{2 o}} \\
& =\quad\left\{\text { SOS definition of StoCCS } ; \text {; Def. of } \mathbf{r t}_{\langle a \mid \bar{a}\rangle}\right\} \\
& \mathbf{r t}_{\langle a \mid \bar{a}\rangle}\left(P_{1}\left|P_{2}, Q_{1}\right| Q_{2}\right)
\end{aligned}
$$

Moreover, the assumption that $P_{1}$ performs the input $a$ and $P_{2}$ performs the output $\bar{a}$ implies $\mathbf{r t}_{\langle\bar{a} \mid a\rangle}\left(P_{1}\left|P_{2}, Q_{1}\right| Q_{2}\right)=0$ and this completes the proof.

\section{Multiplicative rate approach}

The proof is similar to that of the minimum rate case.

\section{D.4 Proof of Proposition 7.5}

Proposition 7.5. For all $P \in \mathcal{P}_{S t o C C S_{A P}}, \alpha \in \mathcal{L}_{S t o C C S_{A P}}$, and $\mathscr{P} \in \mathbf{T F}\left(\mathcal{P}_{S t o C C S_{A P}}, \mathbb{R}_{\geq 0}\right)$, if $P \stackrel{\alpha}{\longmapsto} \mathscr{P}$ can be derived using the set of rules consisting of rules (NIL) and (CHO) of Figure 3, rules (OUT1), (OUT2) and (PAR1) in Figure 9, rules (IN1 $)$, (IN2 $\left.2_{\mathrm{P}}\right)$ in Figure 12 and rule $\left(\mathrm{PAR} 2_{\mathrm{AP}}\right)$ of Figure 13 , then $\mathscr{P} \in \mathbf{F T F}\left(\mathcal{P}_{\text {StoCCS }}, \mathbb{R}_{\geq 0}\right)$.

Proof. Also in this case the assert can easily be proved by induction on the length of the derivation for $P \stackrel{\alpha}{\longmapsto} \mathscr{P}$, using Proposition 5.1.

\section{D.5 Proof of Theorem 7.7}

Theorem 7.7. $\mathcal{R}_{\text {StoCCS }_{A P}}$ is total and deterministic.

Proof. By induction on the structure. The proof is similar to that of Theorem 6.3.

\section{D.6 Proof Theorem 7.8}

Theorem 7.8. For all $P, Q, R \in \mathcal{P}_{\text {StoCCS }_{A P}},(P \mid Q)|R \sim P|(Q \mid R)$

Proof. The statement follows by proving that the following is a bisimulation in $\mathcal{R}_{S t o C C S_{A P}}$.

$$
\begin{aligned}
\mathcal{E}= & \left\{\langle P|(Q \mid R),(P \mid Q)| R\rangle \mid P, Q, R \in \mathcal{P}_{\text {StoCCS }_{A P}}\right\} \\
& \cup\left\{\langle(P \mid Q)|R, P|(Q \mid R)\rangle \mid P, Q, R \in \mathcal{P}_{\text {StoCCS }_{A P}}\right\} \\
& \cup\left\{\langle P|(Q \mid R), P|(Q \mid R)\rangle \mid P, Q, R \in \mathcal{P}_{S_{S t o C C S} A_{A P}}\right\} \\
& \cup\left\{\langle(P \mid Q)|R,(P \mid Q)| R\rangle \mid P, Q, R \in \mathcal{P}_{\text {StoCCS }_{A P}}\right\} \\
& \cup\left\{\langle P, P\rangle \mid P \in \mathcal{P}_{\text {StoCCS }_{A P}}\right\}
\end{aligned}
$$

Indeed, if we prove that $\mathcal{E}$ is a bisimulation, we have that $\mathcal{E} \subseteq \sim$. Moreover, for each

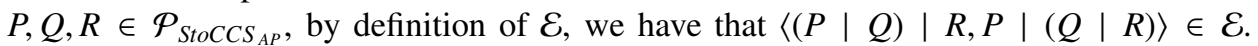
Therefore, for each $P, Q, R \in \mathcal{P}_{\text {StoCCS }_{A P}},(P \mid Q)|R \sim P|(Q \mid R)$.

To prove that $\mathcal{E}$ is a bisimulation, first we have to prove that $\mathcal{E}$ is an equivalence relation. It is easy to prove that:

- for each $P,\langle P, P\rangle \in \mathcal{E}$;

$-\langle P, Q\rangle \in \mathcal{E} \Rightarrow\langle Q, P\rangle \in \mathcal{E}$

ACM Computing Surveys - In Press, Vol. , No., November 2012. 
$-\langle P, Q\rangle \in \mathcal{E} \wedge\langle Q, R\rangle \in \mathcal{E} \Rightarrow\langle P, R\rangle \in \mathcal{E}$

We have to prove that if $\langle P, Q\rangle \in \mathcal{E}$ then for each equivalence class $C$ of $\mathcal{E}$ :

$$
P \stackrel{\alpha}{\rightarrow} \mathscr{P} \Longrightarrow Q \stackrel{\alpha}{\rightrightarrows} \mathscr{Q} \wedge \boxplus \mathscr{P} C=\boxplus \mathscr{Q} C
$$

We can distinguish three cases:

$$
\begin{aligned}
& -P=Q \\
& \Rightarrow \quad\left\{\mathcal{R}_{\text {StoCCS }_{A P}} \text { is fully stochastic }\right\} \\
& P \stackrel{\alpha}{\longmapsto} \mathscr{P} \wedge Q \stackrel{\alpha}{\mapsto} \mathscr{Q} \Longrightarrow \mathscr{P}=\mathscr{Q} \\
& \Rightarrow \quad\{\text { Def. }(\boxplus \mathscr{P} C)\} \\
& \text { 刃 } \mathscr{P} C=\boxplus \mathscr{Q} C \\
& -P=\left(P_{1} \mid P_{2}\right) \mid P_{3} \text { and } Q=P_{1} \mid\left(P_{2} \mid P_{3}\right) \text { : } \\
& -\alpha \neq \delta_{\widehat{a}}^{e} \\
& \Rightarrow \quad\left\{\text { Def. } \mapsto \text { where } P_{i} \stackrel{\alpha}{\longmapsto} \mathscr{P}_{i}\right\} \\
& \mathscr{P}=\left(\left(\mathscr{P}_{1} \otimes_{\mid}\left(\mathcal{X}_{\mathbb{R}_{z 0}} P_{2}\right)\right)+\left(\left(\mathcal{X}_{\mathbb{R}_{20}} P_{1}\right) \otimes_{\mid} \mathscr{P}_{2}\right)\right) \otimes_{\mid}\left(\mathcal{X}_{\mathbb{R}_{\geq 0}} P_{3}\right)+\left(\mathcal{X}_{\mathbb{R}_{\geq 0}}\left(P_{1} \mid P_{2}\right)\right) \otimes_{\mid} \mathscr{P}_{3} \\
& \Rightarrow \quad \text { Def. } \mapsto\} \\
& Q \stackrel{\alpha}{\mapsto} \mathscr{P}_{1} \otimes_{\mid}\left(\mathcal{X}_{\mathbb{R}_{20}}\left(P_{1} \mid P_{2}\right)\right)+\left(\mathcal{X}_{\mathbb{R}_{\geq 0}} P_{1}\right) \otimes_{\mid}\left(\mathscr{P}_{2} \otimes_{\mid}\left(\mathcal{X}_{\mathbb{R}_{20}} P_{3}\right)\right)+\left(\mathcal{X}_{\mathbb{R}_{\geq 0}} P_{1}\right) \otimes_{\mid}\left(\left(\mathcal{X}_{\mathbb{R}_{z 0}} P_{2}\right) \otimes_{\mid}\right. \\
& \mathscr{P}_{3} \text { ) } \\
& \Rightarrow \quad\{C=\{X|(Y \mid Z),(X \mid Y)| Z\}\} \\
& \text { 刃⿻ } \mathscr{P} C= \begin{cases}\mathscr{P}_{1}(X) & Y=P_{2} \wedge Z=P_{3} \\
\mathscr{P}_{2}(Y) & X=P_{1} \wedge Z=P_{3} \\
\mathscr{P}_{3}(Z) & X=P_{1} \wedge Y=P_{2}\end{cases} \\
& \text { ⿴囗十 } C= \begin{cases}\mathscr{P}_{1}(X) & Y=P_{2} \wedge Z=P_{3} \\
\mathscr{P}_{2}(Y) & X=P_{1} \wedge Z=P_{3} \\
\mathscr{P}_{3}(Z) & X=P_{1} \wedge Y=P_{2}\end{cases} \\
& \Rightarrow \\
& \boxplus \mathscr{P} C=\boxplus \mathscr{Q} C \\
& -\alpha=\delta_{\widehat{a}}^{e} \\
& \Rightarrow \quad\{\text { Def. } \rightarrow\}
\end{aligned}
$$

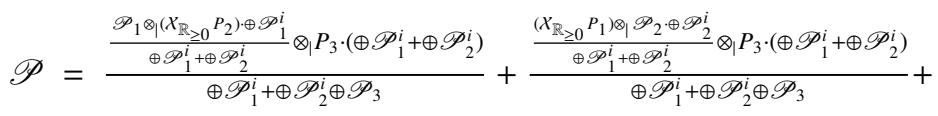

$$
\begin{aligned}
& \frac{\frac{\mathscr{P}_{1}^{i} \otimes_{1} \mathscr{P}^{o}}{\oplus \mathscr{P}_{1}^{i}+\oplus \mathscr{P}_{2}^{i}} \otimes_{1}\left(\mathcal{X}_{\mathbb{R}_{\geq 0}} P_{3}\right) \cdot\left(\oplus \mathscr{P}_{1}^{i}+\oplus \mathscr{P}_{2}^{i}\right)}{\oplus \mathscr{P}_{1}^{i}+\oplus \mathscr{P}_{2}^{i} \oplus \mathscr{P}_{3}}+\frac{\frac{\mathscr{P}_{1}^{o} \otimes_{1} \mathscr{P}^{i}}{\oplus \mathscr{P}_{1}^{i}+\oplus \mathscr{P}_{2}^{i}} \otimes_{1}\left(\mathcal{X}_{\mathbb{R}_{\geq 0}} P_{3}\right) \cdot\left(\oplus \mathscr{P}_{1}^{i}+\oplus \mathscr{P}_{2}^{i}\right)}{\oplus \mathscr{P}_{1}^{i}+\oplus \mathscr{P}_{2}^{i}+\oplus \mathscr{P}_{3}^{o}}+ \\
& \frac{\left(\mathcal{X}_{\mathbb{R}_{\geq 0}}\left(P_{1} \mid P_{2}\right)\right) \otimes_{\mid} \mathscr{P}_{3} \cdot \oplus \mathscr{P}_{3}^{i}}{\oplus \mathscr{P}_{1}^{i}+\oplus \mathscr{P}_{2}^{i}+\oplus \mathscr{P}_{3}^{i}}+\frac{\left(\mathscr{P}_{1}^{i} \otimes_{\mid}\left(\mathcal{X}_{\mathbb{R}_{\geq 0}} P_{2}\right)+\left(\mathcal{X}_{\mathbb{R}_{\geq 0}} P_{1}\right) \otimes_{\mid} \mathscr{P}_{2}^{i}\right) \otimes_{\mid} \mathscr{P}_{3}^{o}}{\oplus \mathscr{P}_{1}^{i}+\oplus \mathscr{P}_{2}^{i} \oplus \mathscr{P}_{3}^{i}}+ \\
& \frac{\left(\mathscr{P}_{1}^{o} \otimes_{\mid}\left(\mathcal{X}_{\mathbb{R}_{\geq 0}} P_{2}\right)+\left(\mathcal{X}_{\mathbb{R}_{\geq 0}} P_{1}\right) \otimes_{\mid} \mathscr{P}_{2}^{o}\right) \otimes_{\mid} \mathscr{P}_{3}^{i}}{\oplus \mathscr{P}_{1}^{i}+\oplus \mathscr{P}_{2}^{i} \oplus \mathscr{P}_{3}^{i}}
\end{aligned}
$$




$$
\begin{aligned}
& =\frac{\left(\left(\mathscr{P}_{1} \otimes_{\mid}\left(X_{\mathbb{R}_{\geq 0}} P_{2}\right)\right) \otimes_{\mid}\left(X_{\mathbb{R}_{\geq 0}} P_{3}\right)\right) \cdot \oplus \mathscr{P}_{1}^{i}}{\oplus \mathscr{P}_{1}^{i}+\oplus \mathscr{P}_{2}^{i} \oplus \mathscr{P}_{3}}+\frac{\left(\left(\left(X_{\mathbb{R}_{\geq 0}} P_{1}\right) \otimes_{\mid} \mathscr{P}_{2}\right) \otimes_{\mid}\left(\mathcal{X}_{\mathbb{R}_{\geq 0}} P_{3}\right)\right) \cdot \oplus \mathscr{P}_{2}^{i}}{\oplus \mathscr{P}_{1}^{i}+\oplus \mathscr{P}_{2}^{i} \oplus \mathscr{P}_{3}}+ \\
& \frac{\left(\mathscr{P}_{1}^{i} \otimes_{\mid} \mathscr{P}^{o}\right) \otimes_{\mid}\left(X_{\mathbb{R}_{\geq 0}} P_{3}\right)}{\oplus \mathscr{P}_{1}^{i}+\oplus \mathscr{P}_{2}^{i} \oplus \mathscr{P}_{3}}+\frac{\left(\mathscr{P}_{1}^{o} \otimes_{1} \mathscr{P}^{i}\right) \otimes_{\mid}\left(X_{\mathbb{R}_{\geq 0}} P_{3}\right)}{\oplus \mathscr{P}_{1}^{i}+\oplus \mathscr{P}_{2}^{i}+\oplus \mathscr{P}_{3}^{o}}+\frac{\left(X_{\mathbb{R}_{\geq 0}}\left(P_{1} \mid P_{2}\right)\right) \otimes_{\mid} \mathscr{P}_{3} \cdot \oplus \mathscr{P}_{3}^{i}}{\oplus \mathscr{P}_{1}^{i}+\oplus \mathscr{P}_{2}^{i}+\oplus \mathscr{P}_{3}^{i}}+ \\
& \frac{\left(\mathscr{P}_{1}^{i} \otimes_{\mid}\left(X_{\mathbb{R}_{\geq 0}} P_{2}\right)+\left(\mathcal{X}_{\mathbb{R}_{\geq 0}} P_{1}\right) \otimes_{\mid} \mathscr{P}_{2}^{i}\right) \otimes_{\mid} \mathscr{P}_{3}^{o}}{\oplus \mathscr{P}_{1}^{i}+\oplus \mathscr{P}_{2}^{i} \oplus \mathscr{P}_{3}^{i}}+\frac{\left(\mathscr{P}_{1}^{o} \otimes_{\mid}\left(\mathcal{X}_{\mathbb{R}_{\geq 0}} P_{2}\right)+\left(\mathcal{X}_{\mathbb{R}_{\geq 0}} P_{1}\right) \otimes_{\mid} \mathscr{P}_{2}^{o}\right) \otimes_{\mid} \mathscr{P}_{3}^{i}}{\oplus \mathscr{P}_{1}^{i}+\oplus \mathscr{P}_{2}^{i} \oplus \mathscr{P}_{3}^{i}} \\
& =\frac{\left(\left(\mathscr{P}_{1} \otimes_{\mid}\left(X_{\mathbb{R}_{\geq 0}} P_{2}\right)\right) \otimes_{\mid}\left(X_{\mathbb{R}_{\geq 0}} P_{3}\right)\right) \cdot \oplus \mathscr{P}_{1}^{i}}{\oplus \mathscr{P}_{1}^{i}+\oplus \mathscr{P}_{2}^{i} \oplus \mathscr{P}_{3}}+\frac{\left(\left(\left(X_{\mathbb{R}_{\geq 0}} P_{1}\right) \otimes_{1} \mathscr{P}_{2}\right) \otimes_{\mid}\left(X_{\mathbb{R}_{\geq 0}} P_{3}\right)\right) \cdot \oplus \mathscr{P}_{2}^{i}}{\oplus \mathscr{P}_{1}^{i}+\oplus \mathscr{P}_{2}^{i} \oplus \mathscr{P}_{3}}+ \\
& \frac{\left(\mathscr{P}_{1}^{i} \otimes_{\mid} \mathscr{P}^{o}\right) \otimes_{\mid}\left(\mathcal{X}_{\mathbb{R}_{\geq 0}} P_{3}\right)}{\oplus \mathscr{P}_{1}^{i}+\oplus \mathscr{P}_{2}^{i} \oplus \mathscr{P}_{3}}+\frac{\left(\mathscr{P}_{1}^{o} \otimes_{1} \mathscr{P}^{i}\right) \otimes_{\mid}\left(\mathcal{X}_{\mathbb{R}_{\geq 0}} P_{3}\right)}{\oplus \mathscr{P}_{1}^{i}+\oplus \mathscr{P}_{2}^{i}+\oplus \mathscr{P}_{3}^{o}}+\frac{\left(\mathcal{X}_{\mathbb{R}_{\geq 0}}\left(P_{1} \mid P_{2}\right)\right) \otimes_{\mid} \mathscr{P}_{3} \cdot \oplus \mathscr{P}_{3}^{i}}{\oplus \mathscr{P}_{1}^{i}+\oplus \mathscr{P}_{2}^{i}+\oplus \mathscr{P}_{3}^{i}}+ \\
& \frac{\left(\mathscr{P}_{1}^{i} \otimes_{\mid}\left(\mathcal{X}_{\mathbb{R}_{\geq 0}} P_{2}\right)\right) \otimes_{\mid} \mathscr{P}_{3}^{o}}{\oplus \mathscr{P}_{1}^{i}+\oplus \mathscr{P}_{2}^{i} \oplus \mathscr{P}_{3}^{i}}+\frac{\left(\left(\mathcal{X}_{\mathbb{R}_{\geq 0}} P_{1}\right) \otimes_{\mid} \mathscr{P}_{2}^{i}\right) \otimes_{\mid} \mathscr{P}_{3}^{o}}{\oplus \mathscr{P}_{1}^{i}+\oplus \mathscr{P}_{2}^{i} \oplus \mathscr{P}_{3}^{i}}+\frac{\left(\mathscr{P}_{1}^{o} \otimes_{\mid}\left(\mathcal{X}_{\mathbb{R}_{20}} P_{2}\right)\right) \otimes_{\mid} \mathscr{P}_{3}^{i}}{\oplus \mathscr{P}_{1}^{i}+\oplus \mathscr{P}_{2}^{i} \oplus \mathscr{P}_{3}^{i}}+\frac{\left(\left(\mathcal{X}_{\mathbb{R}_{\geq 0}} P_{1}\right) \otimes_{\mid} \mathscr{P}_{2}^{o}\right) \otimes_{1} \mathscr{P}_{3}^{i}}{\oplus \mathscr{P}_{1}^{i}+\oplus \mathscr{P}_{2}^{i} \oplus \mathscr{P}_{3}^{i}}
\end{aligned}
$$

where:

$$
P_{j} \stackrel{\delta_{\vec{a}}^{e}}{\rightarrow} \mathscr{P}_{j}, P_{j} \stackrel{\delta_{a}^{e}}{\rightarrow} \mathscr{P}_{j}^{i}, P_{j} \stackrel{\delta_{\bar{a}}^{e}}{\rightarrow} \mathscr{P}_{j}^{o}
$$$$
\Rightarrow \quad\{\text { Def. } \mapsto\}
$$

$$
\begin{aligned}
& \mathscr{Q}=\frac{\mathscr{P}_{1} \otimes_{\mid}\left(\mathcal{X}_{\mathbb{R}_{\geq 0}}\left(P_{2} \mid P_{3}\right)\right) \cdot \oplus \mathscr{P}_{1}^{i}}{\oplus \mathscr{P}_{1}^{i}+\oplus \mathscr{P}_{2}^{i} \oplus \mathscr{P}_{3}}+\frac{\left(X_{\mathbb{R}_{\geq 0}} P_{1}\right) \otimes_{\mid}\left(\mathscr{P}_{2} \otimes_{\mid}\left(\mathcal{X}_{\mathbb{R}_{\geq 0}} P_{3}\right)\right) \cdot \oplus \mathscr{P}_{2}^{i}}{\oplus \mathscr{P}_{1}^{i}+\oplus \mathscr{P}_{2}^{i} \oplus \mathscr{P}_{3}}+ \\
& \frac{\mathscr{P}_{1}^{i} \mid\left(\mathscr{P}^{o} \mid\left(\mathcal{X}_{\mathbb{R}_{\geq 0}} P_{3}\right)\right)}{\oplus \mathscr{P}_{1}^{i}+\oplus \mathscr{P}_{2}^{i} \oplus \mathscr{P}_{3}}+\frac{\mathscr{P}_{1}^{o} \mid\left(\mathscr{P}^{i} \mid\left(\mathcal{X}_{\mathbb{R}_{\geq 0}} P_{3}\right)\right)}{\oplus \mathscr{P}_{1}^{i}+\oplus \mathscr{P}_{2}^{i}+\oplus \mathscr{P}_{3}^{o}}+\frac{\left(X_{\mathbb{R}_{\geq 0}} P_{1}\right) \otimes_{\mid}\left(\left(X_{\mathbb{R}_{\geq 0}} P_{2}\right) \otimes_{\mid} \mathscr{P}_{3}\right) \cdot \oplus \mathscr{P}_{3}^{i}}{\oplus \mathscr{P}_{1}^{i}+\oplus \mathscr{P}_{2}^{i}+\oplus \mathscr{P}_{3}^{i}}+ \\
& \frac{\mathscr{P}_{1}^{i} \mid\left(\left(X_{\mathbb{R}_{\geq 0}} P_{2}\right) \otimes_{\mid} \mathscr{P}_{3}^{o}\right)}{\oplus \mathscr{P}_{1}^{i}+\oplus \mathscr{P}_{2}^{i} \oplus \mathscr{P}_{3}^{i}}+\frac{\left(X_{\mathbb{R}_{\geq 0}} P_{1}\right) \otimes_{\mid}\left(\mathscr{P}_{2}^{i} \otimes_{\mid} \mathscr{P}_{3}^{o}\right)}{\oplus \mathscr{P}_{1}^{i}+\oplus \mathscr{P}_{2}^{i} \oplus \mathscr{P}_{3}^{i}}+\frac{\mathscr{P}_{1}^{o} \otimes_{\mid}\left(\left(X_{\mathbb{R}_{\geq 0}} P_{2}\right) \otimes_{\mid} \mathscr{P}_{3}^{i}\right)}{\oplus \mathscr{P}_{1}^{i}+\oplus \mathscr{P}_{2}^{i} \oplus \mathscr{P}_{3}^{i}}+\frac{\left(X_{\mathbb{R}_{\geq 0}} P_{1}\right) \otimes_{\mid}\left(\mathscr{P}_{2}^{o} \otimes_{\mid} \mathscr{P}_{3}^{i}\right)}{\oplus \mathscr{P}_{1}^{i}+\oplus \mathscr{P}_{2}^{i} \oplus \mathscr{P}_{3}^{i}} \\
& \Rightarrow \quad\{C=\{X|(Y \mid Z),(X \mid Y)| Z\}\} \\
& \boxplus \mathscr{P} C=\boxplus \mathscr{Q} C
\end{aligned}
$$

$-P=X \mid(Y \mid Z)$ and $Q=(X \mid Y) \mid Z$ : follows like the previous one.

\section{D.7 Proof Theorem 7.9}

The semantics we considered in Sect. 7.1 can be modified to obtain associativity of the CCS parallel composition. For this purpose we have to replace $\left(\mathrm{PAR}_{\mathrm{AA}-\mathrm{min}}\right)$ by the following:

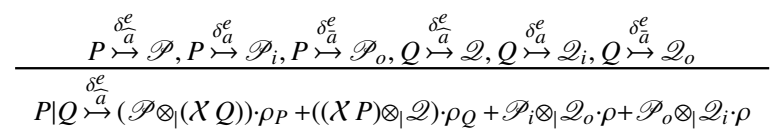

where $\rho_{Q}, \rho_{P}$ are the values used to recompute the rate of synchronizations occurring in $P$ and $Q$, while $\rho$ is used to compute synchronization rates.

$$
\begin{aligned}
& \rho_{P} \quad=_{\text {def }} \quad \frac{\operatorname{MIN}\left\{\oplus \mathscr{P}_{i}+\oplus \mathscr{Q}_{i}, \oplus \mathscr{P}_{o}+\oplus \mathscr{Q}_{o}\right\}}{\operatorname{MIN}\left\{\oplus \mathscr{P}_{i}, \oplus \mathscr{P}_{o}\right\}} \cdot \frac{\oplus \mathscr{P}_{i}}{\oplus \mathscr{P}_{i}+\oplus \mathscr{Q}_{i}} \cdot \frac{\oplus \mathscr{P}_{o}}{\oplus \mathscr{P}_{o}+\oplus \mathscr{Q}_{o}} \\
& \rho_{Q} \quad=_{\text {def }} \quad \frac{\operatorname{MIN}\left\{\oplus \mathscr{P}_{i}+\oplus \mathscr{Q}_{i}, \oplus \mathscr{P}_{o}+\oplus \mathscr{Q}_{o}\right\}}{\operatorname{MIN}\left\{\oplus \mathscr{Q}_{i}, \oplus \mathscr{Q}_{o}\right\}} \cdot \frac{\oplus \mathscr{Q}_{i}}{\oplus \mathscr{P}_{i}+\oplus \mathscr{Q}_{i}} \cdot \frac{\oplus \mathscr{Q}_{o}}{\oplus \mathscr{P}_{o}+\oplus \mathscr{Q}_{o}} \\
& \rho \quad=_{\text {def }} \quad \frac{\operatorname{MIN}\left\{\oplus \mathscr{P}_{i}+\oplus \mathscr{Q}_{i}, \oplus \mathscr{P}_{o}+\oplus \mathscr{Q}_{o}\right\}}{\left(\oplus \mathscr{P}_{i}+\oplus \mathscr{Q}_{i}\right) \cdot\left(\oplus \mathscr{P}_{o}+\oplus \mathscr{Q}_{o}\right)} \cdot \frac{\oplus \mathscr{Q}_{i}}{\oplus \mathscr{P}_{i}+\oplus \mathscr{Q}_{i}} \cdot \frac{\oplus \mathscr{Q}_{o}}{\oplus \mathscr{P}_{o}+\oplus \mathscr{Q}_{o}}
\end{aligned}
$$

The proof of the associativity theorem below proceeds like in the case of Theorem 7.8. 
Theorem 7.9. For all $P, Q, R \in \mathcal{P}_{S_{t o C C S}},(P \mid Q)|R \sim P|(Q \mid R)$

\section{E. PROOFS RELATED TO SECT. 8}

\section{E.1 Proof of Proposition 8.3}

Proposition 8.3. For all $P \in \mathcal{P}_{I M L_{k}}, \alpha \in \mathcal{L}_{I M L_{k}}$, and $\mathscr{P} \in \mathbf{T F}\left(\mathcal{P}_{I M L_{k}}, \mathbb{R}_{\geq 0} \uplus \mathbb{B}\right)$, if $P \stackrel{\alpha}{\mapsto} \mathscr{P}$ can be derived using the set consisting of rules (CHO) and (CNS) of Figure 3 and of the rules of Figure 14, then the following holds: $\mathscr{P} \in \mathbf{F T F}\left(\mathcal{P}_{I M L_{k}}, \mathbb{R}_{\geq 0} \uplus \mathbb{B}\right)$.

Proof. By induction on the length of the derivation for $P \stackrel{\alpha}{\longmapsto} \mathscr{P}$. The proof is similar to that of of Proposition 6.1.

\section{E.2 Proof of Proposition 8.4}

Proposition 8.4. For all $P \in \mathcal{P}_{I M L_{k}}, \alpha \in \mathcal{L}_{I M L_{k}}$ and $\mathscr{P} \in \mathbf{F T F}\left(\mathscr{P}_{I M L_{k}}, \mathbb{R}_{\geq 0} \uplus \mathbb{B}\right)$ such that $P \stackrel{\alpha}{\longmapsto} \mathscr{P}$ can be derived using the set consisting of rules (CHO) and (CNS) of Figure 3 and of the rules of Figure 14, then the following holds: (i) if $\alpha \in \mathcal{A}$ then $\mathscr{P} \in \mathbf{F T F}\left(\mathcal{P}_{I M L_{k}}, \mathbb{B}\right)$; (ii) if $\alpha=\delta^{e}$ then $\mathscr{P} \in \mathbf{F T F}\left(\mathcal{P}_{I M L_{k}}, \mathbb{R}_{\geq 0}\right)$.

Proof. By induction on the length of the derivation for $P \stackrel{\alpha}{\rightarrow} \mathscr{P}$. Let $n \geq 1$ be the length of the derivation for proving $P \stackrel{\alpha}{\leftrightarrow} \mathscr{P}$.

Base case: Trivial since the only cases in which $P \stackrel{\alpha}{\rightarrow} \mathscr{P}$ can be derived with a proof of length 1 are those for inaction, rate-prefix and action-prefix. In all these cases the assert easily follows from the relevant semantics rules (see Figure 14).

Inductive step: The last assert of any proof of length $n>1$ must be of the form $P+$ $Q \stackrel{\alpha}{\rightarrow} \mathscr{P}+\mathscr{Q}$, or $P \|_{L} Q \stackrel{\alpha}{\longmapsto}\left(\mathscr{P} \otimes_{\|_{L}}^{\alpha}(X \alpha Q)\right)+\left((X \alpha P) \otimes_{\|_{L}}^{\alpha} \mathscr{Q}\right)$, or $X \stackrel{\alpha}{\longmapsto} \mathscr{P}$, or $P \|_{L} Q \stackrel{\alpha}{\rightarrow} \mathscr{P} \otimes_{\|_{L}}^{\alpha} \mathscr{Q}$. We show the proof only for the first two cases, the other being simpler.

Case: $P+Q \stackrel{\alpha}{\mapsto} \mathscr{P}+\mathscr{Q}$

(i) if $\alpha \in \mathcal{A}$, from the definition of the FuTS semantics of $\mathrm{IML}_{k}$ we get $P \stackrel{\alpha}{\rightarrow} \mathscr{P}, Q \stackrel{\alpha}{\mapsto} \mathscr{Q}$ and by the I.H., $\mathscr{P}, \mathscr{Q} \in \mathbf{F T F}\left(\mathcal{P}_{I M L_{k}}, \mathbb{B}\right)$ thus also $\mathscr{P}+\mathscr{Q} \in \mathbf{F T F}\left(\mathcal{P}_{I M L_{k}}, \mathbb{B}\right)$ by definition of $+_{\mathbb{B}}$ and, consequently, of $+\operatorname{over} \operatorname{FTF}\left(\mathcal{P}_{I M L_{k}}, \mathbb{B}\right)$; (ii) if $\alpha=\delta^{e}$ the proof is identical as for case (i), but using $\mathbb{R}_{\geq 0}$ instead of $\mathbb{B}$.

Case: $P \|_{L} Q \stackrel{\alpha}{\longmapsto}\left(\mathscr{P} \otimes_{\|_{L}}^{\alpha}(X \alpha Q)\right)+\left((X \alpha P) \otimes_{\|_{L}}^{\alpha} \mathscr{Q}\right)$

(i) Suppose $\alpha \in \mathcal{A}$. From the definition of the FuTS semantics of $\mathrm{IML}_{k} P \stackrel{\alpha}{\hookrightarrow} \mathscr{P}, Q \stackrel{\alpha}{\mapsto} \mathscr{Q}$, and, by I.H., $\mathscr{P}, \mathscr{Q} \in \mathbf{F T F}\left(\mathcal{P}_{I M L_{k}}, \mathbb{B}\right)$. Moreover, also $(X \alpha Q),(X \alpha P) \in \mathbf{F T F}\left(\mathscr{P}_{I M L_{k}}, \mathbb{B}\right)$ by definition of $\mathcal{X}$, since $\alpha \in \mathcal{A}$. Using the closure properties of $\operatorname{FTF}\left(\mathcal{P}_{I M L_{k}}, \mathbb{B}\right)$, and in particular Proposition 5.1, we get the assert; (ii) if $\alpha=\delta^{e}$ the proof is identical as for case (i), but using $\mathbb{R}_{\geq 0}$ instead of $\mathbb{B}$.

\section{E.3 Proof of Theorem 8.6}

Theorem 8.6. $\mathcal{R}_{I M L_{k}}$ is total and deterministic. 
Proof. By induction on the structure. The proof is the similar to that of Theorem 6.3.

\section{E.4 Proof of Theorem 8.7}

Theorem 8.7. For all $P, Q \in \mathcal{P}_{I M L_{k}}, a \in \mathcal{A}$, and unique functions $\mathscr{P} \in \mathbf{F T F}\left(\mathcal{P}_{I M L_{k}}, \mathbb{B}\right)$ and $\mathscr{P}^{\prime} \in \mathbf{F T F}\left(\mathscr{P}_{I M L_{k}}, \mathbb{R}_{\geq 0}\right)$ such that $P \stackrel{a}{\longmapsto} \mathscr{P}$ and $P \stackrel{\delta^{e}}{\mapsto} \mathscr{P}^{\prime}$ the following holds: (i) $(\mathscr{P} Q)=1_{\mathbb{B}}$ if and only if $P \stackrel{a}{\rightarrow} Q ;\left(\right.$ ii) $\left(\mathscr{P}^{\prime} Q\right)=\operatorname{rt}(P, Q)$.

Proof. Proof of part (i). For the sake of conciseness, we prove both the direct $(\Rightarrow)$ and the reverse $(\Leftarrow)$ implication together. For the direct implication we proceed by induction on the length of the derivation for FUTS semantics $(P \stackrel{a}{\hookrightarrow} \mathscr{P})$, while we use induction on the length of the derivation for the SOS $(P \stackrel{a}{\rightarrow} Q)$ for the reverse implication. Let $n \geq 1$ be the length of the derivation for proving $P \stackrel{a}{\rightarrow} \mathscr{P}(P \stackrel{a}{\rightarrow} Q$, respectively).

Base case: Trivial since the only case in which $P \stackrel{a}{\rightarrow} \mathscr{P}$ can be derived with a proof of length 1 and $(\mathscr{P} Q)=1_{\mathbb{B}}$ is $a . Q \stackrel{a}{\mapsto} \mathscr{P}$ with $\mathscr{P}=\left[Q \mapsto 1_{\mathbb{B}}\right]$. But $a \cdot Q \stackrel{a}{\rightarrow} Q$ in the SOS definition of $\mathrm{IML}_{k}$. On the other hand, the only case in which $P \stackrel{a}{\rightarrow} Q$ can be derived with a proof of length 1 is when $P=a \cdot Q$, in which case $P \stackrel{a}{\longmapsto}\left[Q \mapsto 1_{\mathbb{B}}\right]$.

Inductive step: The last assert of any proof of length $n>1$ must be of the form $P_{1}+P_{2} \stackrel{a}{\longmapsto}$ $\mathscr{P}_{1}+\mathscr{P}_{2}$, or $X \stackrel{a}{\rightarrow} \mathscr{P}_{1}$, or $P_{1} \|_{L} P_{2} \stackrel{a}{\longmapsto}\left(\mathscr{P}_{1} \otimes_{\|_{L}}^{\alpha}\left(X a P_{2}\right)\right)+\left(\left(\mathcal{X} a P_{1}\right) \otimes_{\|_{L}}^{\alpha} \mathscr{P}_{2}\right)$, or $P_{1} \quad \|_{L} \quad P_{2} \stackrel{a}{\rightarrow} \mathscr{P}_{1} \otimes_{\|_{L}}^{\alpha} \mathscr{P}_{2}$ and $P_{1}+P_{2} \stackrel{a}{\rightarrow} Q$, or $X \stackrel{a}{\rightarrow} Q$, or $P_{1} \|_{L} P_{2} \stackrel{a}{\rightarrow} Q$ (with $a \notin L$ ), or $P_{1} \|_{L} P_{2} \stackrel{a}{\rightarrow} Q$ (with $\left.a \in L\right)$, respectively.

Case: $\left\{\begin{array}{l}P_{1}+P_{2} \stackrel{a}{\rightarrow} \mathscr{P}_{1}+\mathscr{P}_{2}, \text { for } \Rightarrow \\ P_{1}+P_{2} \stackrel{a}{\rightarrow} Q, \text { for } \Leftarrow\end{array}\right.$

$$
\left(\mathscr{P}_{1}+\mathscr{P}_{2}\right) Q=1_{\mathbb{B}}
$$

$\stackrel{\text { Def. FUTS semantics of IML }}{\rightleftarrows}$; Def. $\left.\left(\mathscr{P}_{1}+\mathscr{P}_{2}\right)\right\}$

$$
P_{1} \stackrel{a}{\mapsto} \mathscr{P}_{1}, P_{2} \stackrel{a}{\mapsto} \mathscr{P}_{2},\left(\mathscr{P}_{1} Q\right)=1_{\mathbb{B}} \text { or }\left(\mathscr{P}_{2} Q\right)=1_{\mathbb{B}}
$$

$\Rightarrow \quad\{$ I.H. $\}$

$\Leftarrow \quad$ II.H.; Unicity of $\left.\mathscr{P}_{1}, \mathscr{P}_{2}\right\}$

$$
P_{1} \stackrel{a}{\rightarrow} Q \text { or } P_{2} \stackrel{a}{\rightarrow} Q
$$

$\rightleftarrows \quad\left\{\right.$ Def. SOS of $\left.\mathrm{IML}_{k}\right\}$

$$
P_{1}+P_{2} \stackrel{a}{\rightarrow} Q
$$

Case: $\left\{\begin{array}{l}X \stackrel{a}{\rightarrow} \mathscr{P}_{1}, X:=P_{1}, \text { for } \Rightarrow \\ X \stackrel{a}{\rightarrow} Q, X:=P_{1}, \text { for } \Leftarrow\end{array}\right.$

$$
\left(\mathscr{P}_{1} Q\right)=1_{\mathbb{B}}
$$




$$
\begin{aligned}
& \Rightarrow \quad\left\{\text { Def. FuTS semantics of } \mathrm{IML}_{k}\right\} \\
& \Leftarrow \quad \text { Logics }\} \\
& P_{1} \stackrel{a}{\mapsto} \mathscr{P}_{1},\left(\mathscr{P}_{1} Q\right)=1_{\mathbb{B}} \\
& \Rightarrow \quad\{\text { I.H. }\} \\
& \left.\Leftarrow \quad \text { II.H.; Unicity of } \mathscr{P}_{1}\right\} \\
& P_{1} \stackrel{a}{\rightarrow} Q
\end{aligned}
$$

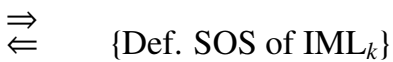

$$
\begin{aligned}
& X \stackrel{a}{\rightarrow} Q
\end{aligned}
$$

Case: $\left\{\begin{array}{l}P_{1} \|_{L} P_{2} \stackrel{a}{\rightarrow}\left(\mathscr{P}_{1} \otimes_{\|_{L}}^{\alpha}\left(\mathcal{X} a P_{2}\right)\right)+\left(\left(X a P_{1}\right) \otimes_{\|_{L}}^{\alpha} \mathscr{P}_{2}\right), \text { for } \Rightarrow \\ P_{1} \|_{L} P_{2} \stackrel{a}{\rightarrow} Q, a \notin L, \text { for } \Leftarrow\end{array}\right.$

$$
\left(\mathscr{P}_{1} \otimes_{\|_{L}}^{\alpha}\left(X a P_{2}\right)\right)+\left(\left(X a P_{1}\right) \otimes_{\|_{L}}^{\alpha} \mathscr{P}_{2}\right) Q=1_{\mathbb{B}}
$$

$\Rightarrow \quad\left\{\right.$ Def. FuTS semantics of $\operatorname{IML}_{k}$; Def. + and $\otimes_{\|_{L}}^{\alpha}$ over $\operatorname{FTF}\left(\mathcal{P}_{I M L_{k}}, \mathbb{B}\right) ;$ Def. $\left.\mathcal{X}\right\}$

$\Leftarrow \quad\left\{\right.$ Def. + and $\otimes_{\|_{L}}^{\alpha}$ over $\operatorname{FTF}\left(\mathcal{P}_{I M L_{k}}, \mathbb{B}\right) ;$ Def. $\left.\mathcal{X}\right\}$

$P_{1} \stackrel{a}{\rightarrow} \mathscr{P}_{1}, P_{2} \stackrel{a}{\longmapsto} \mathscr{P}_{2}, a \notin L$,

$Q=Q_{1} \|_{L} P_{2}$ for some $Q_{1}$ such that $\left(\mathscr{P}_{1} Q_{1}\right)=1_{\mathbb{B}}$, or

$Q=P_{1} \|_{L} Q_{2}$ for some $Q_{2}$ such that $\left(\mathscr{P}_{2} Q_{2}\right)=1_{\mathbb{B}}$

$\Rightarrow \quad\{$ I.H. $\}$

$\Leftarrow \quad$ I.H.; Unicity of $\left.\mathscr{P}_{1}, \mathscr{P}_{2}\right\}$

$Q=Q_{1} \|_{L} P_{2}$ for some $Q_{1}$ such that $P_{1} \stackrel{a}{\rightarrow} Q_{1}, a \notin L$ or

$Q=P_{1} \|_{L} Q_{2}$ for some $Q_{2}$ such that $P_{2} \stackrel{a}{\rightarrow} Q_{2}, a \notin L$

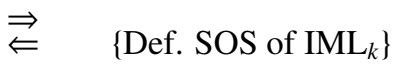

$P_{1} \|_{L} P_{2} \stackrel{a}{\rightarrow} Q, a \notin L$

Case: $\left\{\begin{array}{l}P_{1}\left\|_{L} P_{2} \stackrel{a}{\mapsto} \mathscr{P}_{1}\right\|_{L} \mathscr{P}_{2}, \text { for } \Rightarrow \\ P_{1} \|_{L} P_{2} \stackrel{a}{\rightarrow} Q, a \in L, \text { for } \Leftarrow\end{array}\right.$

$$
\left(\mathscr{P}_{1} \otimes_{\|_{L}}^{\alpha} \mathscr{P}_{2}\right) Q=1_{\mathbb{B}}
$$

$\Rightarrow \quad\left\{\right.$ Def. FuTS semantics of $\operatorname{IML}_{k}$; Def. $\left.\|_{L} \operatorname{over} \operatorname{FTF}\left(\mathcal{P}_{I M L_{k}}, \mathbb{B}\right)\right\}$ 


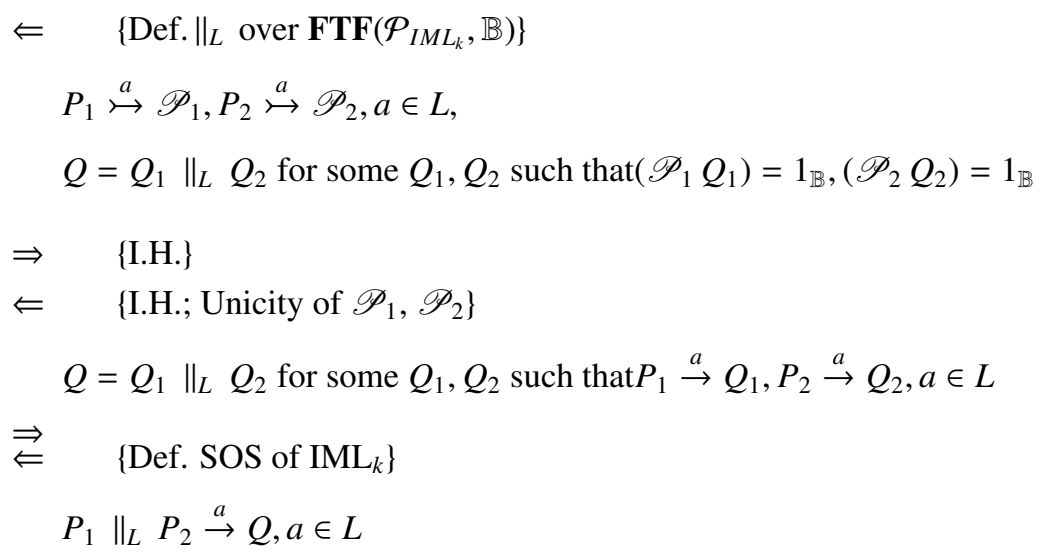

Proof of part (ii). We proceed by induction on the length of the derivation for $P \stackrel{\delta^{e}}{\rightarrow} \mathscr{P}^{\prime}$. Let $n \geq 1$ be the length of the derivation for proving $P \stackrel{\delta^{e}}{\mapsto} \mathscr{P}^{\prime}$.

Base case: Trivial since the only case in which $P \stackrel{\delta^{e}}{\rightarrow} \mathscr{P}^{\prime}$ can be derived with a proof of length 1 and $\left(\mathscr{P}^{\prime} Q\right) \neq 0$ is $\lambda . Q \stackrel{\delta^{e}}{\longmapsto}[Q \mapsto \lambda]$ and $\operatorname{rt}(\lambda . Q, Q)=\lambda$ by the SOS definition of $\mathrm{IML}_{k}$ and the definition of rt. In all other cases, $\left(\mathscr{P}^{\prime} Q\right)=0$ and there are no transitions $\stackrel{\lambda}{P} Q$, hence $\operatorname{rt}(P, Q)=0$ by definition.

Inductive step: The last assert of any proof of length $n>1$ must be of the form $P_{1}+P_{2} \stackrel{\delta^{e}}{\longmapsto}$ $\mathscr{P}_{1}+\mathscr{P}_{2}$, or $X \stackrel{\delta^{e}}{\mapsto} \mathscr{P}_{1}$, or $P_{1} \|_{L} P_{2} \stackrel{\delta^{e}}{\longmapsto}\left(\mathscr{P}_{1} \|_{L}\left(X \delta^{e} P_{2}\right)\right)+\left(\left(X \delta^{e} P_{1}\right) \|_{L} \mathscr{P}_{2}\right)$.

Case: $P_{1}+P_{2} \stackrel{\delta^{e}}{\mapsto} \mathscr{P}_{1}+\mathscr{P}_{2}$

$$
\begin{aligned}
& \left(\mathscr{P}_{1}+\mathscr{P}_{2}\right) Q \\
= & \left\{\text { Def. }\left(\mathscr{P}_{1}+\mathscr{P}_{2}\right)\right\} \\
& \left(\mathscr{P}_{1} Q\right)+\left(\mathscr{P}_{2} Q\right) \\
= & \left\{P_{1} \stackrel{\delta^{e}}{\mapsto} \mathscr{P}_{1}, P_{2} \stackrel{\delta^{e}}{\mapsto} \mathscr{P}_{2} ; \text { I.H. }\right\} \\
& \operatorname{rt}\left(P_{1}, Q\right)+\operatorname{rt}\left(P_{2}, Q\right)
\end{aligned}
$$

$=\quad\left\{\right.$ SOS definition of $\mathrm{IML}_{k}$; Def. of $\left.\mathbf{r t}\right\}$

$$
\operatorname{rt}\left(P_{1}+P_{2}, Q\right)
$$

Case: $X \stackrel{\delta^{e}}{\longmapsto} \mathscr{P}_{1}, X:=P_{1}$

$$
\left(\mathscr{P}_{1} Q\right)
$$

ACM Computing Surveys - In Press, Vol. , No., November 2012. 


$$
\begin{aligned}
= & \quad\left\{P_{1} \stackrel{\delta^{e}}{\mapsto} \mathscr{P}_{1} ; \text { I.H. }\right\} \\
& \mathbf{r t}\left(P_{1}, Q\right) \\
= & \quad\left\{\text { SOS definition of } \mathrm{IML}_{k} ; \text { Def. of } \mathbf{r t}\right\} \\
& \mathbf{r t}(X, Q)
\end{aligned}
$$

Case: $P_{1} \|_{L} P_{2} \stackrel{\delta^{e}}{\mapsto}\left(\mathscr{P}_{1} \otimes_{\|_{L}}^{\alpha}\left(\mathcal{X} \delta^{e} P_{2}\right)\right)+\left(\left(X \delta^{e} P_{1}\right) \otimes_{\|_{L}}^{\alpha} \mathscr{P}_{2}\right)$

We observe that, from the FuTS semantics of $\mathrm{IML}_{k}$, if $Q$ is neither of the form $Q_{1} \|_{L} P_{2}$, nor of the form $P_{1} \|_{L} Q_{2}$ then $\left(\left(\mathscr{P}_{1} \otimes_{\|_{L}}^{\alpha}\left(\mathcal{X} \delta^{e} P_{2}\right)\right)+\left(\left(\mathcal{X} \delta^{e} P_{1}\right) \otimes_{\|_{L}}^{\alpha} \mathscr{P}_{2}\right)\right) Q=0$. On the other hand, we observe that the only $\rightarrow$ transitions allowed by the SOS definition of $\mathrm{IML}_{k}$ are to terms of the form $Q_{1} \|_{L} P_{2}$ or $P_{1} \|_{L} Q_{2}$, so, also $\operatorname{rt}\left(P_{1} \|_{L} P_{2}, Q\right)=0$ if $Q$ is neither of the form $Q_{1} \|_{L} P_{2}$, nor of the form $P_{1} \|_{L} Q_{2}$. Let us assume, w.l.g., $Q$ be of the form $Q_{1} \|_{L} P_{2}$; we have to consider two cases: (a) $Q_{1} \neq P_{1}$, and (b) $Q_{1}=P_{1}$.

Case a):

$$
\begin{aligned}
& \left(\left(\mathscr{P}_{1} \otimes_{\|_{L}}^{\alpha}\left(\mathcal{X} \delta^{e} P_{2}\right)\right)+\left(\left(X \delta^{e} P_{1}\right) \otimes_{\|_{L}}^{\alpha} \mathscr{P}_{2}\right)\right) Q_{1} \|_{L} P_{2} \\
= & \left\{\text { Def. + over } \mathbf{F T F}\left(\mathscr{P}_{I M L_{k}}, \mathbb{R}_{\geq 0}\right)\right\} \\
& \left(\left(\mathscr{P}_{1} \otimes_{\|_{L}}^{\alpha}\left(\mathcal{X} \delta^{e} P_{2}\right)\right) Q_{1} \|_{L} P_{2}\right)+\left(\left(\left(X \delta^{e} P_{1}\right) \otimes_{\|_{L}}^{\alpha} \mathscr{P}_{2}\right) Q_{1} \|_{L} P_{2}\right) \\
= & \quad\left\{\text { Def. } \otimes_{\|_{L}}^{\alpha} ; \text { Def. } \mathcal{X}\right\} \\
& \left(\mathscr{P}_{1} Q_{1}\right) \\
= & \left\{P_{1} \stackrel{\delta^{e}}{\mapsto} \mathscr{P}_{1} ; \text { I.H. }\right\} \\
& \operatorname{rt}\left(P_{1}, Q_{1}\right) \\
= & \left\{\text { SOS definition of IML } \mathrm{IL}_{k} ; \text { Def. of } \mathbf{r t}\right\} \\
& \operatorname{rt}\left(P_{1}\left\|_{L} P_{2}, Q_{1}\right\|_{L} P_{2}\right)
\end{aligned}
$$

Case b):

$$
\begin{aligned}
& \left(\left(\mathscr{P}_{1} \otimes_{\|_{L}}^{\alpha}\left(X \delta^{e} P_{2}\right)\right)+\left(\left(X \delta^{e} P_{1}\right) \otimes_{\|_{L}}^{\alpha} \mathscr{P}_{2}\right)\right) P_{1} \|_{L} P_{2} \\
= & \left\{\text { Def. }+ \text { over } \mathbf{F T F}\left(\mathscr{P}_{I M L_{k}}, \mathbb{R}_{\geq 0}\right)\right\} \\
& \left(\left(\mathscr{P}_{1} \|_{L}\left(X \delta^{e} P_{2}\right)\right) P_{1} \|_{L} P_{2}\right)+\left(\left(\left(X \delta^{e} P_{1}\right) \|_{L} \mathscr{P}_{2}\right) P_{1} \|_{L} P_{2}\right) \\
= & \left\{\text { Def. } \otimes_{\|_{L}}^{\alpha} ; \text { Def. } \mathcal{X}\right\} \\
& \left(\mathscr{P}_{1} P_{1}\right)+\left(\mathscr{P}_{2} P_{2}\right) \\
= & \left\{P_{1} \stackrel{\delta^{e}}{\rightarrow} \mathscr{P}_{1} ; P_{2} \stackrel{\delta^{e}}{\rightarrow} \mathscr{P}_{2} \text { I.H. }\right\}
\end{aligned}
$$


$\operatorname{rt}\left(P_{1}, \mathscr{P}_{1}\right)+\operatorname{rt}\left(P_{2}, \mathscr{P}_{2}\right)$

$=\quad\left\{\right.$ SOS definition of $\mathrm{IML}_{k}$; Def. of rt $\}$

$\operatorname{rt}\left(P_{1}\left\|_{L} P_{2}, P_{1}\right\|_{L} P_{2}\right)$

The proof for the case in which $Q$ is of the form $P_{1} \|_{L} Q_{2}$ is similar.

\section{E.5 Proof of Proposition 8.9}

Proposition 8.9. For all $P \in \mathcal{P}_{M A L}, \alpha \in \mathcal{L}_{M A L}$, and $\mathscr{P} \in \mathbf{T F}\left(\mathcal{P}_{M A L}, \mathbb{R}_{\geq 0}\right)$, if $P \stackrel{\alpha}{\mapsto} \mathscr{P}$ can be derived using the set consisting of rules (CNS) of Fig. 3 and of the rules of Fig. 15, then the following holds: $\mathscr{P} \in \mathbf{F T F}\left(\mathcal{P}_{M A L}, \mathbb{R}_{\geq 0}\right)$.

Proof. By induction on the length of the derivation for $P \stackrel{\alpha}{\hookrightarrow} \mathscr{P}$. The proof is similar to that of Proposition 6.1.

\section{E.6 Proof of Proposition 8.10}

Proposition 8.10. For all $P \in \mathcal{P}_{M A L}, \alpha \in \mathcal{L}_{M A L}$ and $\mathscr{P} \in \mathbf{F T F}\left(\mathcal{P}_{M A L}, \mathbb{R}_{\geq 0}\right)$ such that $P \stackrel{\alpha}{\longmapsto} \mathscr{P}$ can be derived using the set consisting of rules (CNS) of Fig. 3 and of the rules of Fig. 15, if $\alpha \in \Pi_{\mathcal{A}}$ then $\oplus \mathscr{P} \leq 1$.

Proof. The proof is easily done by induction on the length of the derivation for $P \stackrel{\alpha}{\hookrightarrow}$ $\mathscr{P}$, it is similar to that of Proposition 8.4, taking into account the fact that we have that $\oplus \llbracket\left\{p_{1}:: P_{1}+\ldots+p_{h}:: P_{h}\right\} \rrbracket \leq 1$ by construction, and is thus left out here.

\section{E.7 Proof of Theorem 8.12}

Theorem 8.12. $\mathcal{R}_{M A L}$ is total.

Proof. By induction on the structure. The proof is similar to that of Theorem 6.3. 


\section{REFERENCES}

Aceto, L., FoKking, W., AND Verhoef, C. 2001. Structural Operational Semantics. In Handbook of Process Algebra. Elsevier, 197-292.

Aldini, A., Bernardo, M., and Corradini, F. 2010. A Process Algebraic Approach to Software Architecture Design. Springer.

BARTELS, F. 2002. GSOS for probabilistic transition systems (extened abstract). ENTCS 65, 1. Elsevier, 1-25.

Bartels, F., Sokolova, A., AND de Vink, E. 2003. A hierarchy of probabilistic system types. ENTCS 82, 1. Elsevier, 1-19.

Bergstra, J., Ponse, A., Smolka, S., Eds. 2001. Handbook of Process Algebra. Elsevier.

Bernardo, M. 2010. On the Expressiveness of Markovian Process Calculi with Durational and Durationless Actions. In GANDALF 2010, EPTCS 25, 199-213.

Bernardo, M., De Nicola, R., and Loreti, M. 2010. Uniform labeled transition systems for nondeterministic, probabilistic, and stochastic processes. In TGC 2010, LNCS 6084. Springer, 35-56.

Blom, S., Fokkink, W., Groote, J. F., van Langevelde, I., Lisser, B., and van de Pol, J. 2001. $\mu$ crl: A toolset for analysing algebraic specifications. In CAV 2001, LNCS 2102. Springer, 250-254.

Bohnenkamp, H., D’Argenio, P., Hermanns, H., and Katoen, J.-P. 2006. MOdEST: A Compositional Modeling Formalism for Hard and Softly Timed Systems. IEEE TSE 32, 10. IEEE Computer Society Press, 812-830.

Brinksma, E. and Hermanns, H. 2001. Process Algebra and Markov Chains. In Lectures on Formal Methods and Performance Analysis, LNCS 2090. Springer, 181-231.

BuchHolz, P. 1994. Markivian Process Algebra: Composition and Equivalence. In Proceedings of PAPM '94, Erlangen, 11-30.

Cardelli, L. and Mardare, R. 2010. The Measurable Space of Stochastic Processes. In QEST 2010. IEEE Computer Society Press, 171-180.

Dang Van Hung and Zhou Chaochen. 1999. Probabilistic Duration Calculus for Continuous Time. Formal Aspects of Computing. The International Journal of Formal Methods. 11. Springer, 21-44.

De Nicola, R., Katoen, J.-P., Latella, D., Loreti, M., and Massink, M. 2006. Klaim and its Stochastic Semantics. Technical Report 6, Dipartimento di Sistemi e Informatica, Università di Firenze. (available at http://rap.dsi.unifi.it/ ^loreti/papers/TR062006.pdf).

De Nicola, R., Latella, D., Loreti, M., and Massink, M. 2009a. MarCaSPiS: a Markovian Extension of a Calculus for Services. In SOS 2008, ENTCS 229. Elsevier, 11-26.

De Nicola, R., Latella, D., Loreti, M., and Massink, M. 2009b. On a Uniform Framework for the Definition of Stochastic Process Languages. In FMICS 2009, LNCS 5825. Springer, 9-25.

De Nicola, R., Latella, D., Loreti, M., and Massink, M. 2009c. Rate-based Transition Systems for Stochastic Process Calculi. In Automata, Languages and Programming. Part II, LNCS 5556. Springer, 435-446.

De Nicola, R., Latella, D., and Massink, M. 2005. Formal modeling and quantitative analysis of KLAIM-based mobile systems. In SAC 2005. ACM, 428-435.

Deng, Y., van Glabbeek, R., Hennessy, M., Morgan, C., and Zhang, C. 2007. Characterising testing preorders for finite probabilistic processes. In LICS. IEEE, Computer Society Press, 313-325.

Eisentraut, C., Hermanns, H., and Zhang, L. 2010. On Probabilistic Automata in Continuous Time. In IEEE Symposium on Logic in Computer Science. IEEE, Computer Society Press, 342-351.

Fahrenberg, U., Thrane, C., and Larsen, K. 2011. Distances for weighted transition systems: Games and properties. In QAPL 2011 EPTCS 57, 134-147.

van Glabbeek, R. 2001. The Linear Time - Branching Time Spectrum I. In Handbook of Process Algebra. Elsevier, 3-99.

Gotz, N., Herzog, U., and Rettelbach, M. 1993. Multiprocessor and distributed systems design: The integration of functional specification and performance analysis using stochastic process algebras. In Performance Evaluation of Computer and Communication Systems. LNCS 729. Springer, 121-146.

HeRmanns, H. 2002. Interactive Markov Chains. LNCS 2428. Springer.

Hermanns, H., Herzog, U., and Katoen, J.-P. 2002. Process algebra for performance evaluation. Theoretical Computer Science 274, 1-2. Elsevier, 43-87.

Hermanns, H., Herzog, U., and Mertsiotakis, V. 1998. Stochastic process algebras - between LOTOS and Markov chains. Computer Networks and ISDN Systems 30 North-Holland, 901-924. 
Hermanns, H. and Johr, S. 2007. Uniformity by Construction in the Analysis of Nondeterministic Stochastic Systems. In DSN 2007. IEEE Computer Society Press, 718-728.

Hermanns, H. and Katoen, J. 2010. The How and Why of Interactive Markov Chains. In FMCO. LNCS 6286. Springer, 311-337.

HiLlston, J. 1994. The nature of synchronisation. In PAPM '94. 51-70. Erlangen, Germany - ISSN 0344-3515.

HiLlston, J. 1996. A compositional approach to performance modelling. Distinguished Dissertation in Computer Science. Cambridge University Press.

HoJjat, H., Reza, Mousavi, M., and SiRJani, M. 2008. A framework for performance evaluation and functional verification in stochastic process algebras. In SAC 2008. ACM, 339-346

Kurn, B. 2009. Structural Operational Semantics for Weighted Transition Systems. In Semantics and Algebraic Specification. LNCS 5700. Springer-Verlag, 121-139.

Kuin, B. and Sassone, V. 2008. Structural Operational Semantics for Stochastic Process Calculi. In FoSSaCS 2008. LNCS 4962. Springer-Verlag, 428-442.

Knast, R. 1969. Continuous-Time Probabilistic Automata. Information and Control 15. Elsevier, 335-352.

Latella, D., Massink, M., and De Vink. 2012. Bisimulation of Labeled State-to-Function Transition Systems of Stochastic Process Languages. In ACCAT 2012. EPTCS 93, 23-43.

Lijun Zhang, Hermanns, H., Eisenbrand, F., and Jansen, D. 2008. Flow Faster: Efficient Decision Algorithms For Probabilistic Simulations. Logical Methods in Computer Science 4, 6, 1-43.

MilneR, R. 1989. Communication and Concurrency. Series in Computer Science. Prentice Hall.

Neuts, M. 1981. Matrix-geometric Solutions in Stochastic Models-An Algorithmic Approach. The Johns Hopkins University Press, Baltimore.

Plotkin, G. D. 2004. A structural approach to operational semantics. J. Log. Algebr. Program. 60-61. Elsevier, 17-139.

Priami, C. 1995. Stochastic $\pi$-Calculus. The Computer Journal 38, 7. Oxford University Press, 578-589.

Segala, R. 1995. Modeling and verification of randomized distributed real-time systems. Ph.D Thesis, MIT.

Sokolova, A. AND de VinK, E. 2004. Probabilistic Automata: System Types, Parallel Composition and Comparison. In Validation of Stochastic Systems. A Guide to Current Research. LNCS 2925. Springer, 1-43.

Timmer, M., Katoen, J-P., van de Pol, J., and Stoelinga, M. 2012. Efficient Modelling and Generation of Markov Automata. In CONCUR 2012. LNCS 7454. Springer-Verlag, 364-379. 


\section{Institution Markets \\ IMT}

INSTITUTE FOR ADVANCED STUDIES LUCCA

2012 ( ) IMT Institute for Advanced Studies, Lucca Piazza San ponziano 6, 5100 Lucca, Italy. www.imtlucca.it 